\title{
Generalized Degrees Freedom of Noncoherent MIMO Channels with Asymmetric Link Strengths
}

\author{
Joyson Sebastian, Suhas N. Diggavi
}

\begin{abstract}
We study the generalized degrees of freedom $(\mathrm{gDoF})$ of block-fading noncoherent multiple input multiple output (MIMO) channels with asymmetric distributions of link strengths and a coherence time of $T$ symbol durations. We derive the optimal signaling structure for communication for the asymmetric MIMO channel, which is distinct from that for the MIMO channel with independent and identically distributed (i.i.d.) links. We extend the existing results for the single input multiple output (SIMO) channel with i.i.d. links to the asymmetric case, proving that selecting the statistically best antenna is gDoF-optimal. Using the gDoF result for the SIMO channel, we prove that for $T=1$, the gDoF is zero for MIMO channels with arbitrary link strengths. We show that selecting the statistically best antenna is gDoF-optimal for the multiple input single output (MISO) channel. We also derive the gDoF for the $2 \times 2$ MIMO channel with different exponents in the direct and cross links. In this setting, we show that it is always necessary to use both the antennas to achieve the $\mathrm{gDoF}$, in contrast to the results for the $2 \times 2$ MIMO channel with i.i.d. links. We show that having weaker crosslinks, gives gDoF gain compared to the case with i.i.d. links. For the noncoherent MIMO channel with i.i.d. links, the traditional method of training each transmit antenna independently is degrees of freedom (DoF) optimal, whereas we observe that for the asymmetric $2 \times 2$ MIMO channel, the traditional training is not gDoF-optimal. We extend this observation to a larger $M \times M$ MIMO channel by demonstrating a strategy that can achieve larger $\mathrm{gDoF}$ than a traditional training-based method.
\end{abstract}

\section{INTRODUCTION}

The capacity of fading multiple input multiple output (MIMO) channels when neither the receiver nor the transmitter knows the fading coefficients was first studied by Marzetta and 
Hochwald [2]. They considered a block fading channel model where the fading gains are independent and identically distributed (i.i.d.) Rayleigh random variables and remain constant for $T$ symbol periods. In [3], Zheng and Tse introduced the idea of communication over a Grassmanian manifold for the noncoherent MIMO channel and derived the capacity behavior when the links are i.i.d. and the signal-to-noise-ratio (SNR) is high. Their characterization was tight for the capacity at large SNR, when the coherence time was large compared to the number of antennas. In [4], this tight characterization was extended to the case when the number of antennas was large compared to the coherence time.

Some works have especially considered the case with coherence time $T=1$. The noncoherent single input single output (SISO) channel with $T=1$ was considered by Taricco and Elia [5]. They obtained the capacity behavior in asymptotically low and high SNR regimes. The noncoherent SISO channel with $T=1$ was further studied by Abou-Faycal et al. [6] and they showed that for any given SNR, the capacity is achieved by an input distribution with a finite number of mass points. For the noncoherent MIMO channel with $T=1$ and high SNR, Lapidoth and Moser [7] showed that the capacity behaves double logarithmically with SNR.

When a complete capacity characterization of a wireless network is difficult to obtain, the notions of degrees of freedom $(\mathrm{DoF})$ and generalized degrees of freedom $(\mathrm{gDoF})$ can be used to understand the asymptotic behavior of the capacity. For example, for a point-to-point network parameterized by channel strengths ${ }^{1} \rho_{1}^{2}, \rho_{2}^{2}, \ldots, \rho_{L}^{2}$ on its links, the complete capacity characterization obtains the capacity for all values of $\rho_{1}^{2}, \rho_{2}^{2}, \ldots, \rho_{L}^{2}$. The DoF characterization finds the asymptotic behavior of the prelog of capacity along the line $\log \left(\rho_{1}^{2}\right)=\log \left(\rho_{2}^{2}\right)=\cdots=\log \left(\rho_{L}^{2}\right)$ in the $L$-dimensional space of link strengths in $\mathrm{dBm}$. The gDoF characterization is more general; it finds the asymptotic behavior of the prelog of capacity along the line $\left(\log \left(\rho_{1}^{2}\right) / \gamma_{1}\right)=$ $\left(\log \left(\rho_{2}^{2}\right) / \gamma_{2}\right)=\cdots=\left(\log \left(\rho_{L}^{2}\right) / \gamma_{L}\right)$ with constants $\gamma_{1}, \ldots, \gamma_{L}$. Equivalently, for the gDoF characterization, we can set $\log \left(\rho_{1}^{2}\right) / \gamma_{1}=\log \left(\rho_{2}^{2}\right) / \gamma_{2}=\cdots=\log \left(\rho_{L}^{2}\right) / \gamma_{L}=\log (\mathrm{SNR})$ and let SNR $\rightarrow \infty$. The gDoF characterization was first used in [8] to characterize the asymptotic behavior of the capacity region of a 2-user symmetric interference channel (IC) for high SNR. There the link strengths were set to scale as SNR, SNR ${ }^{\alpha}, \mathrm{SNR}^{\alpha}, \mathrm{SNR}$ for the 4 links of the IC. This method of scaling the channel strengths with different SNR exponents to obtain the gDoF

\footnotetext{
${ }^{1}$ For ease of analysis, we absorb the transmit SNR into the channel strengths, hence the capacity characterization does not include the transmit SNR explicitly. The noise at the receivers are assumed to be of unit variance.
} 
region is also done in other works like [9], [10].

The DoF characterization has been used to study the noncoherent MIMO channel with temporal correlation within each fading block. In [11], Morgenshtern et al. studied the single input multiple output (SIMO) channel with temporally correlated Rayleigh block-fading and showed that the SIMO channel can have a larger DoF than the SISO channel, under some mild assumptions on the temporal correlation. The noncoherent MIMO channel with temporally correlated block fading was studied in [12]. There it was shown that the noncoherent MIMO channel with temporally correlated block fading can have a larger DoF than the noncoherent MIMO channel with constant block fading.

Some works have studied noncoherent networks (with more than two nodes) for the capacity behavior at high SNR. In [13], it was shown that for noncoherent networks with $T=1$, the gDoF is zero; this was an extension of the result for the noncoherent MIMO channel with $T=1$ from [7]. Koch and Kramer studied the noncoherent single relay network [14] and showed that under certain conditions on the fading statistics, the relay does not increase the capacity at high SNR. In [15], the noncoherent MIMO full-duplex single relay channel with block-fading was studied, and it was shown that Grassmanian signaling can achieve the DoF without using the relay. Also, the results in [15] show that for certain regimes, decode-and-forward with Grassmanian signaling can approximately achieve the capacity at high SNR.

To the best of our knowledge, the existing works consider a DoF framework for studying the noncoherent channels, i.e., the links in the network scale with the same SNR exponent. However, in networks, the links could have asymmetry in the channel strengths. In this case, a gDoF framework could better capture the system behavior. We consider the noncoherent MIMO channel with asymmetric link strengths as a first step in the direction of studying the asymmetric noncoherent networks.

\section{A. Contributions and Outline}

In this paper, we consider a noncoherent channel model with coherence time of $T$ symbol periods and asymmetric link distributions, where the link strengths are scaled with different exponents of SNR. In essence, we are moving from the DoF-framework in [2], [3] to the generalized DoF of noncoherent MIMO channels.

Next generation wireless architecture envisages dense deployment of access points [16]. Another architectural proposal is to use cloud radio access networks (CRAN) [17]. These imply that 
multiple access points could be connected through a (reliable) backhaul. The implication of this is that of widely separated antennas, which form a virtual antenna array. Such widely separated antennas could be used for coordinated transmission and reception, e.g., coordinated multipoint COMP [18]. These widely separated antennas could have disparate average strengths motivating our model (especially SIMO channels and MISO channels). This is illustrated in Figure 1.

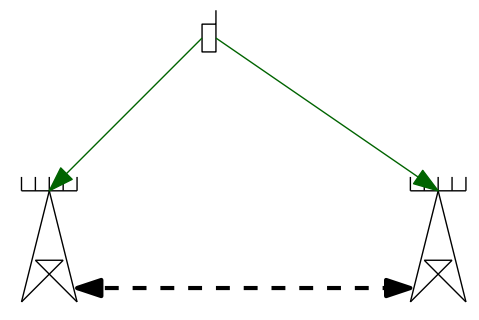

SIMO

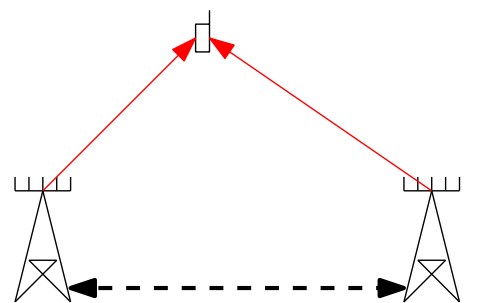

MISO

Figure 1. Noncoherent SIMO channels and MISO channels with asymmetric statistics can arise in COMP architecture where multiple basestations can cooperate through the backhaul.

The MIMO case arises when the receiver could be widely spread (see Figure 2) as would be the case when users can cooperate using a separate sidechannel [19].

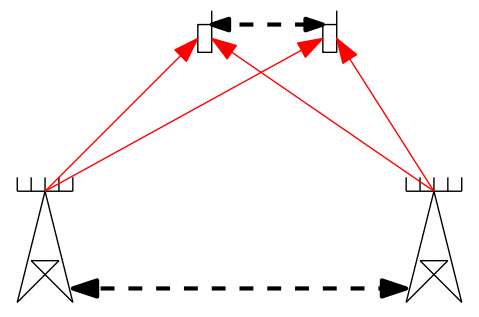

MIMO

Figure 2. Noncoherent MIMO channel with asymmetric statistics can arise with COMP architecture and device-to-device cooperation.

Another motivation for this model comes from the study of networks. Here one can think of the cut-set as a distributed MIMO channel (see Figure 3) where the nodes are widely separated again resulting in this model. 


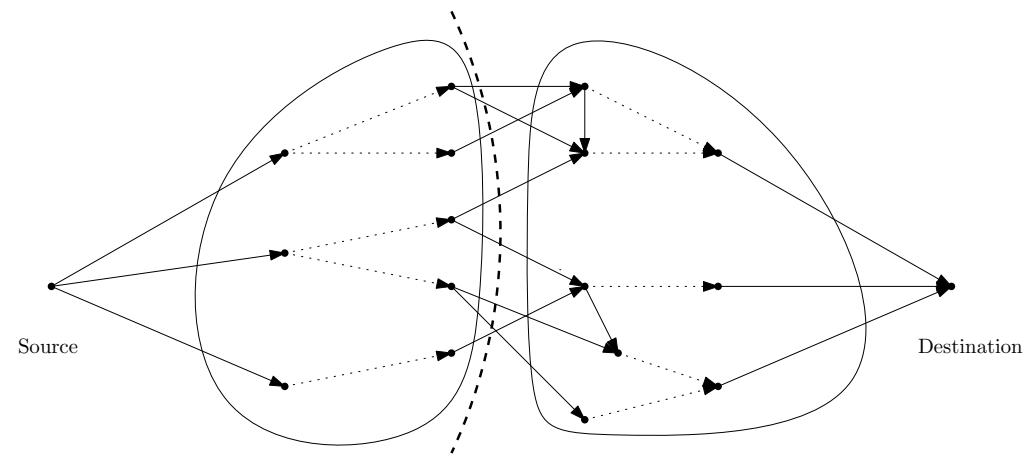

Figure 3. Noncoherent MIMO channel with asymmetric statistics can arise in the analysis of noncoherent networks.

The asymmetric case is also motivated by a fundamental question about the robustness of the results in [2], [3] to the changes in the i.i.d. channel model.

For our channel model with arbitrary (fading) link strengths, we show in Theorem 1 that the capacity achieving input distribution is of the form $L Q$ where $L$ is a lower triangular matrix and $Q$ is an isotropically distributed unitary matrix independent of $L$. This is in contrast to the result for the i.i.d. setting, where the capacity achieving distribution has the form $D Q$ with a diagonal matrix $D$ [2]. In Theorem 4, we demonstrate that the gDoF of a SIMO channel can be achieved by retaining only the signal received by the best receive antenna. The gDoF result for the SIMO channel is used in Theorem 5 to show that for $T=1$, the $\mathrm{gDoF}$ is always zero for a MIMO channel of any size. In Theorem 6, we show that the gDoF of the MISO channel can be achieved by signaling only over the statistically best transmit antenna.

In a setting with $N$ receive antennas, when the exponents in the SNR-scaling are same for all the links (i.i.d. setting), the number of transmit antennas $M$, required to attain the optimal DoF was shown to be $\min (\lfloor T / 2\rfloor, N)$ [3]. It was shown that increasing the number of transmit antennas beyond this value reduces the DoF. In this paper, we provide evidence that this is not the case when the SNR exponents are different: in Theorem 7, we show that for a $2 \times 2$ MIMO channel with different SNR exponents in the direct and cross links, and $T=2$, both the transmit antennas are required to achieve the $\mathrm{gDoF}$. We also show that having smaller exponents in crosslinks lead to a gDoF gain of $(2 / T) \gamma_{\text {diff }}$ compared to the case with same SNR exponents in all the links, where $\gamma_{\text {diff }}$ is the difference in the SNR exponents. In showing this, several novel techniques were needed. In particular, we would like to highlight the technique used in Lemma 22. where in the outer bound optimization problem, we show that using a discrete probability distribution with a single mass point is gDoF optimal. To obtain this result, we discretized the 
input distribution without a loss in $\mathrm{gDoF}$, and subsequently used linear programming arguments to show that there exists an optimal distribution with just one mass point. We believe that our techniques for the $2 \times 2 \mathrm{MIMO}$ channel provide intuitions for studying larger noncoherent networks, especially in analyzing the cut-sets.

Traditional training-based schemes for MIMO systems allocate a training symbol to train each transmit antenna independently. Our results for the $2 \times 2 \mathrm{MIMO}$ channel also demonstrate that a traditional training-based scheme is not gDoF-optimal. Our scheme has a gDoF gain of $(2 / T) \gamma_{\text {diff }}$ compared to a training-based scheme. We also numerically evaluate the rates achievable using our scheme in some specific scenarios and compare it to the traditional training-based schemes. For a $2 \times 2$ system with coherence time $T=2$, transmit SNR $=23 \mathrm{~dB}$, direct links with average strength 0.1 and crosslinks with average strength 0.025 , our noncoherent scheme can obtain a $7 \%$ gain in the rate ${ }^{2}$ compared to the schemes that use a symbol for training. We demonstrate more rate points and the gains in Table $\Pi$ on page 14 . In Theorem 8 , we extend our observation on the nonoptimality of traditional training-based schemes to larger $M \times M$ MIMO channels with given SNR exponents on the direct and cross links, where we demonstrate a strategy that can achieve larger gDoF than a training-based scheme.

Extending our outer bounds to the general MIMO channel seems a difficult task at the moment; the LQ transformation process used for deriving the outer bound for the $2 \times 2 \mathrm{MIMO}$ channel as done in (46), 47), 48, 49) and the subsequent Lemmas (Lemma 19, Lemma 20 and Lemma 21 for bounding the terms in those equations do not easily extend to $3 \times 3$ or higher MIMO channels.

Outline: The rest of this paper is organized as follows: in Section II, we give the notations and set up the system model; Section III presents our main results, and Section IV provides analysis and proofs for the results in Section III. Some details of the proofs are deferred to the Appendixes. In Section V, we give our conclusions and final remarks.

\section{NOTATION AND SYSTEM MODEL}

\section{A. Notational Conventions}

We use the notation $\mathcal{C N}\left(\mu, \sigma^{2}\right)$ for circularly symmetric complex Gaussian distribution with mean $\mu$ and variance $\sigma^{2}$. We use the symbol $\sim$ with overloaded meanings: one to indicate that a

\footnotetext{
${ }^{2}$ The channel strengths in this example are given without absorbing the transmit SNR into them. The noise is assumed to be of unit variance.
} 
random variable has a given distribution and second to indicate that two random variables have the same distribution. The logarithm to base 2 is denoted by $\log ()$. The notation $A^{\dagger}$ indicates the Hermitian conjugate of a matrix $A$ and $\operatorname{Tran}(A)$ indicates the transpose of $A$.

The gDoF characterization for a point-to-point network with different link strengths $\rho_{1}^{2}, \rho_{2}^{2}, \ldots, \rho_{L}^{2}$ captures the asymptotic behavior of the capacity along the curve $\log \left(\rho_{1}^{2}\right) / \gamma_{1}=\log \left(\rho_{2}^{2}\right) / \gamma_{2}=$ $\cdots=\log \left(\rho_{L}^{2}\right) / \gamma_{L}$ for any given constants $\gamma_{1}, \ldots, \gamma_{L}$ as

$$
\operatorname{gDoF}_{\gamma_{1}, \ldots, \gamma_{L}}=\lim _{\log \left(\rho_{1}^{2}\right) / \gamma_{1}=\log \left(\rho_{2}^{2}\right) / \gamma_{2}=\cdots=\log \left(\rho_{L}^{2}\right) / \gamma_{L}=\log (\mathrm{SNR}), \mathrm{SNR} \rightarrow \infty} \frac{C\left(\rho_{1}^{2}, \rho_{2}^{2}, \ldots, \rho_{L}^{2}\right)}{\log (\mathrm{SNR})},
$$

where $C\left(\rho_{1}^{2}, \rho_{2}^{2}, \ldots, \rho_{L}^{2}\right)$ is the capacity of the network for a given value of channel strengths $\rho_{1}^{2}, \rho_{2}^{2}, \ldots, \rho_{L}^{2}$. We use the notation $\doteq$ for relative equality, i.e., we say

$$
f_{1}(\mathrm{SNR}) \doteq f_{2}(\mathrm{SNR})
$$

if

$$
\lim _{\mathrm{SNR} \rightarrow \infty} \frac{f_{1}(\mathrm{SNR})}{\log (\mathrm{SNR})}=\lim _{\mathrm{SNR} \rightarrow \infty} \frac{f_{2}(\mathrm{SNR})}{\log (\mathrm{SNR})} .
$$

The notations $\dot{\leq}, \geq$ are defined analogously. The script $\mathcal{P}$ is used to indicate an optimization problem and $(\mathcal{P})$ is used to denote the optimal value of the objective function. We use the overloaded notation

$$
\operatorname{gDoF}(\mathcal{P})=\lim _{\mathrm{SNR} \rightarrow \infty} \frac{(\mathcal{P})}{\log (\mathrm{SNR})}
$$

to indicate the scaling of the optimal value of $\mathcal{P}$ when the optimization problem depends on the SNR.

\section{B. System Model}

We consider a block-fading MIMO channel with $M$ transmit and $N$ receive antennas, and a coherence time of $T$ symbol durations. The signal flow (over a blocklength $T$ ) is given by:

$$
Y=G X+W
$$

where $X$ is the $M \times T$ matrix of transmitted symbols with rows $\underline{X_{i}}$ corresponding to each transmit antenna; $G$ represents the $N \times M$ channel matrix (which is independently generated every $T$ symbols), and its elements $g_{i j}$ are independent with $g_{i j} \sim \mathcal{C N}\left(0, \rho_{i j}^{2}\right)=\mathcal{C N}\left(0, \mathrm{SNR}^{\gamma_{i j}}\right)$, where the exponents $\gamma_{i j}$ are (constant) parameters of the MIMO channel. For convenience, we also use the notation $\underline{\rho}^{2}(n)$ to denote the row vector of channel strengths to $n^{\text {th }}$ receiver antenna. The 
columns of $G$ are denoted by $\overline{g_{i}}$, and these correspond to channels from each transmit antenna. The variable $Y$ represents the $N \times T$ matrix of received symbols, with rows corresponding to each receive antenna and $W$ is an $N \times T$ noise matrix with i.i.d. elements $w_{i j} \sim \mathcal{C N}(0,1)$. The transmit signals have the average power constraint:

$$
\frac{1}{M T} \sum_{m=1}^{M} \sum_{t=1}^{T} \mathbb{E}\left[\left|x_{m t}\right|^{2}\right]=1 .
$$

\section{MAIN RESULTS}

In this section, we go through the main results of our paper. We first look at the general results for the noncoherent MIMO channel with asymmetric link strengths. In Theorem 1, we prove a structural result for the optimizing distribution for the noncoherent MIMO channel. This result has some similarities to that for the noncoherent MIMO channel with i.i.d. links in the sense that part of the structure is similar. We then consider a noncoherent MIMO channel that can be decomposed into smaller disjoint channels. In this case, the channel matrix is a block diagonal matrix. We prove similar to the coherent case, that the power can be allocated across the disjoint parts and coding can be done separately among the disjoint parts to achieve the capacity. This result is proved in Theorem 2. This result can be used to derive the gDoF of noncoherent parallel channels. This is stated as Corollary 3.

Then we look at noncoherent MIMO channels with specific structures. In Theorem 4, we consider the noncoherent SIMO channel and derive its gDoF. For this case, we prove that the gDoF is achieved by using the statistically best antenna. The gDoF result for the SIMO channel can be used to prove that the $\mathrm{gDoF}$ is zero for any MIMO channel when $T=1$. We obtain this by decomposing the MIMO channel into different SIMO channels. We obtain this result in Theorem 5. Next, we consider the noncoherent MISO channel and prove a similar result, that its gDoF can be achieved using the statistically best antenna. This is proved in Theorem 6 .

The next specific structure we look at is the noncoherent $2 \times 2 \mathrm{MIMO}$ channel with a given SNR exponent in the direct links and another SNR exponent in the crosslinks. We handle this case in Theorem 7. We observe that standard training-based schemes are not gDoF-optimal for $2 \times 2$ MIMO channels in general. In Theorem 8 , we extend this observation to larger $M \times M$ MIMO channels. 


\section{A. Results for General Noncoherent MIMO Channels}

Theorem 1. The capacity of a noncoherent MIMO system can be achieved with input signal $X$ of the form $X=L Q$ with $L$ being a lower triangular matrix and $Q$ being an isotropically distributed unitary matrix independent of $L$.

Proof: The proof is given in Section IV-B.

This theorem is in contrast with the result for the case when the elements of $G$ and $W$ are i.i.d. Gaussian. In that case, the structure of an optimal $X$ could be written as $X=D Q$ where $D$ is diagonal [2]. In our system model, only $W$ has i.i.d. elements which ends up restricting the structure to the form $L Q$.

Theorem 2. Let the channel matrix $G$ of the MIMO system be block diagonal as $G=\operatorname{diag}\left(G_{1}, \ldots, G_{K}\right)$ where $G_{i}$ are the diagonal blocks of $G$, then the capacity $C\left(P\right.$, diag $\left.\left(G_{1}, \ldots, G_{K}\right)\right)$ of the channel for a power $P$ can be achieved by splitting the power across the blocks: $C\left(P\right.$, diag $\left.\left(G_{1}, \ldots, G_{K}\right)\right)=$ $\max _{P_{1}+\cdots+P_{K} \leq P}\left(C\left(P_{1}, G_{1}\right)+\cdots+C\left(P_{K}, G_{K}\right)\right)$.

Proof idea: This result holds for the coherent MIMO channel and the proof for noncoherent case is similar. We just need to show $C\left(P, \operatorname{diag}\left(G_{1}, G_{2}\right)\right)=\max _{P_{1}+P_{2} \leq P}\left(C\left(P_{1}, G_{1}\right)+C\left(P_{2}, G_{2}\right)\right)$ because of induction. Let $X_{G 1}, X_{G 2}$ be the transmitted symbols in the parts $G_{1}$ and $G_{2}$ of the channel. Similarly $Y_{G 1}, Y_{G 2}$ be the corresponding received symbols. Now $I(X ; Y) \leq I\left(X_{G 1} ; Y_{G 1}\right)+$ $I\left(X_{G 2} ; Y_{G 2}\right)$ because $\left(X_{G 2}, Y_{G 2}\right)-X_{G 1}-Y_{G 1},\left(X_{G 1}, Y_{G 1}\right)-X_{G 2}-Y_{G 2}$ are Markov chains and the desired result easily follows. The detailed steps are given in Appendix C

Now we have the following corollary from the above theorem.

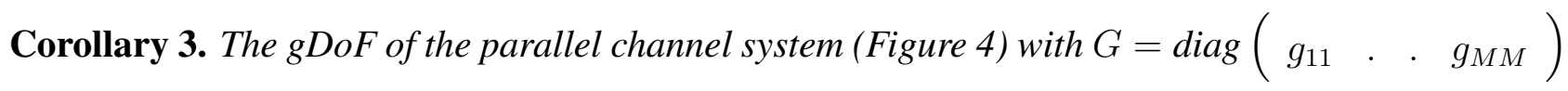
and links $g_{i i} \sim \mathcal{C N}\left(0, \rho_{i i}^{2}\right)=\mathcal{C N}\left(0, \mathrm{SNR}^{\gamma_{i i}}\right)$ is $\sum_{i}\left(1-\frac{1}{T}\right) \gamma_{i i}$.

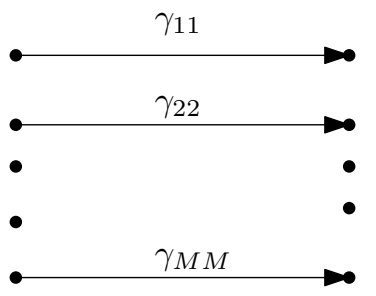

Figure 4. Parallel channels with given SNR exponents. 


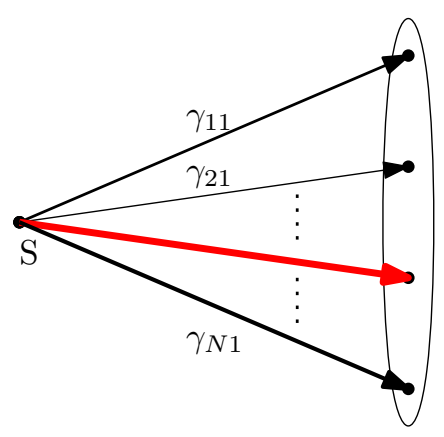

$\mathrm{D}$

Figure 5. Selecting the statistically best antenna is gDoF-optimal for the noncoherent SIMO channel with given SNR exponents.

Proof: For a SISO channel with link $g_{i i}$ distributed according to $\mathcal{C N}\left(0, \rho_{i i}^{2}\right)=\mathcal{C N}\left(0, \mathrm{SNR}^{\gamma_{i i}}\right)$, the $\mathrm{gDoF}$ is $\left(1-\frac{1}{T}\right) \gamma_{i i}$ [3]. The result for the parallel channel system follows by decomposing the parallel channel into individual SISO channels and using Theorem 2.

\section{B. SIMO Channels and MISO Channels}

In this subsection, we consider noncoherent SIMO channels and MISO channels with asymmetric link strengths. The gDoF result for the SIMO channel can be easily derived by extending the results for the case with i.i.d. links. For $T=1$, the gDoF result for the SIMO channel can be extended to the arbitrary MIMO case. For the MISO case, the existing techniques are not sufficient for computing the outer bound. We develop new techniques, manipulating entropy expressions using linear algebra techniques to derive the gDoF of the MISO channel.

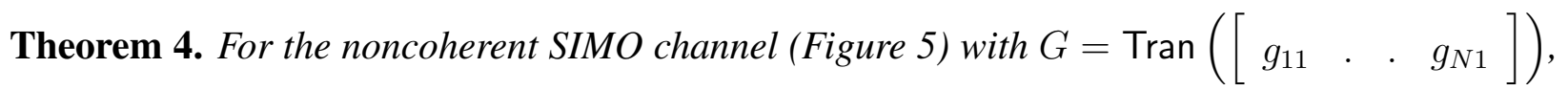
where $g_{i 1} \sim \mathcal{C N}\left(0, \rho_{i 1}^{2}\right)=\mathcal{C N}\left(0, \mathrm{SNR}^{\gamma_{i 1}}\right)$, the $g D o F$ is $\left(1-\frac{1}{T}\right) \max _{i} \gamma_{i 1}$, i.e., the gDoF can be achieved by using only the statistically best receive antenna.

Proof: We only need to prove the outer bound, since the achievability follows by using the statistically best receive antenna. The outer bound can be proved as an extension of results for the SIMO channel with i.i.d. links. We construct another SIMO channel with a larger capacity than the given asymmetric SIMO channel. Let $\rho_{*}^{2}=\max _{i} \rho_{i 1}^{2}$. Now with $W$ being a $T \times 1$ noise vector with i.i.d. $\mathcal{C N}(0,1)$ elements, $G^{\prime}$ being a $1 \times N$ channel matrix with i.i.d. $\mathcal{C N}\left(0, \rho_{*}^{2}\right)$ elements, $W_{1}$ being a noise vector with independent (but not identical) Gaussian elements $w_{1 i} \sim$ $\mathcal{C N}\left(0, \rho_{*}^{2} / \rho_{1 i}^{2}-1\right), i \in\{1,2, \ldots, T\}$ and $K$ being a constant diagonal matrix with elements 
$k_{i i}=\rho_{1 i} / \rho_{*}, i \in\{1,2, \ldots, T\}$, we observe that $K\left(G^{\prime} X+W+W_{1}\right)$ has the same distribution as

$$
Y=G X+W .
$$

Hence by the data processing inequality $I(X ; G X+W) \leq I\left(X ; G^{\prime} X+W\right)$. Now due to the results for i.i.d. noncoherent MIMO channels [3], we have $I\left(X ; G^{\prime} X+W\right) \leq(T-1) \log \left(\rho_{*}^{2}\right)$. Hence the required result follows.

Using the above result for the SIMO channel, we can now prove that the gDoF is zero for any MIMO channel for $T=1$.

Theorem 5. ( $g D o F$ of arbitrary MIMO channel for $T=1$ ) For any $G$ with $T=1$, the $g D o F$ is zero.

Proof: This can be shown by separately examining the SIMO channels constructed using $\overline{g_{i}} i \in\{1,2, \ldots, N\}$ from $G=\left[\begin{array}{llll}\overline{g_{1}} & \overline{g_{2}} & . & . \overline{g_{N}}\end{array}\right]$ and $\underline{X_{i}}$ from $X=\operatorname{Tran}\left[\operatorname{Tran}\left(\underline{X_{1}}\right) \ldots \operatorname{Tran}\left(\underline{X_{N}}\right)\right]$, $G$ being the channel and $X$ being the symbols for the whole MIMO channel. Consider $N$ SIMO channels $Y_{i}=\overline{g_{i}} \underline{X_{i}}+\frac{W_{i}}{\sqrt{N}}$, where $W_{i}$ and $W$ have the same distribution but are independent. Now

$$
I(X ; G X+W) \leq I\left(X ; \overline{g_{1}} \underline{X_{1}}+\frac{W_{1}}{\sqrt{N}}, \ldots, \overline{g_{N}} \underline{X_{N}}+\frac{W_{N}}{\sqrt{N}}\right)
$$

using the data processing inequality since $\sum_{i=1}^{N}\left(W_{i} / \sqrt{N}\right) \sim W$ and $\sum_{i=1}^{N} \overline{g_{i}} \underline{X_{i}}=G X$. This creates a new channel which is decomposable into $N$ SIMO channels, and the new channel has a larger capacity than the original channel. Hence the required result follows due to Theorem 2 by decomposing the new channel into $N$ SIMO channels and using the fact that each SIMO channel has zero gDoF for $T=1$ (due to Theorem 4).

Note that the above result is a generalization of the zero DoF result for MIMO channels by Lapidoth and Moser [7]. In their model, the channel statistics is fixed and the power of the i.i.d. noise is scaled. However, our result is more general in the sense that we allow the fading channel strengths to be scaled with different exponents.

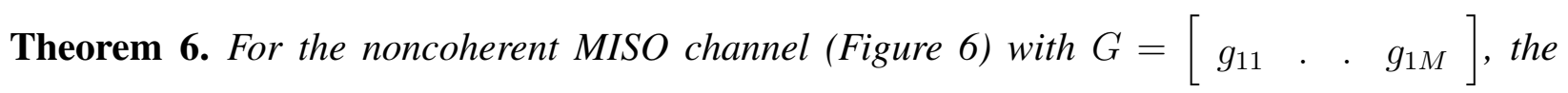
gDoF is $\left(1-\frac{1}{T}\right) \max _{i} \gamma_{1 i}$, i.e., the gDoF can be achieved by only using the statistically best transmit antenna. 


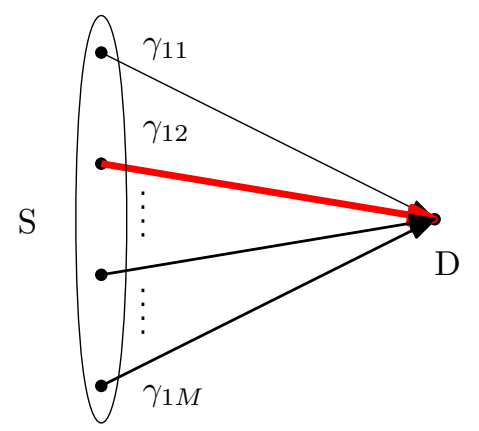

Figure 6. Selecting the statistically best antenna is gDoF-optimal for a noncoherent MISO channel with given SNR exponents.

Proof idea: We only need to prove the outer bound. In this case, $Y$ is a column vector and $h(Y)$ can be evaluated using Lemma 13 . Also, we prove that

$$
h(Y \mid X) \geq \mathbb{E}\left[\log \left(1+\sum_{i=1}^{M} \rho_{1 i}^{2}\left\|\underline{X_{i}}\right\|^{2}\right)\right]
$$

using linear algebra techniques. With these two results, the gDoF result follows. See Section IV-C for details.

\section{The $2 \times 2$ MIMO Channel}

In this subsection, we describe the results for the $2 \times 2$ MIMO channel with SNR exponents $\gamma_{D}$ in the direct links and $\gamma_{C L}$ in the crosslinks (Figure 7). This is one of the simple extensions starting from the MIMO channel with i.i.d. links and this extension demonstrates different properties than the i.i.d. case. We describe our outer bound and obtain a signaling distribution to solve the outer bound optimization problem in terms of $\mathrm{gDoF}$. The signaling distribution for achievability uses the structure of our solution to the outer bound optimization problem.

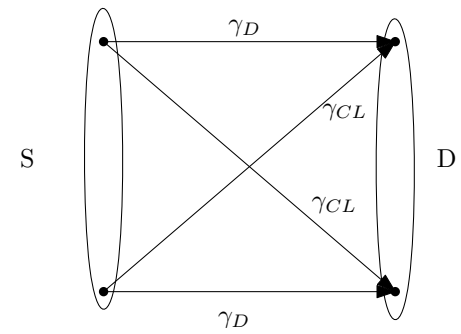

Figure 7. The $2 \times 2 \mathrm{MIMO}$ channel with SNR exponents $\gamma_{D}$ in the direct links and $\gamma_{C L}$ in the crosslinks. 
Theorem 7. For the $2 \times 2$ noncoherent MIMO channel with

$$
G=\left[\begin{array}{ll}
g_{11} & g_{12} \\
g_{21} & g_{22}
\end{array}\right]
$$

where $g_{11} \sim g_{22} \sim \mathcal{C N}\left(0, \mathrm{SNR}^{\gamma_{\mathrm{D}}}\right), g_{12} \sim g_{21} \sim \mathcal{C N}\left(0, \mathrm{SNR} \gamma_{\mathrm{CL}}\right)$ and $\gamma_{D} \geq \gamma_{C L}$, the gDoF is given in Table $\square$ and can be achieved by

$$
X=\left[\begin{array}{cccccc}
a & 0 & 0 & . & . & 0 \\
\eta & c & 0 & . & . & 0
\end{array}\right] Q
$$

where $\eta \sim \mathcal{C N}\left(0,|b|^{2}\right),|a|^{2}=\mathrm{SNR}^{-\gamma_{a}},|b|^{2}=\mathrm{SNR}^{-\gamma_{\mathrm{b}}},|c|^{2}=\mathrm{SNR}^{-\gamma_{c}}$ with the values of $\left(\gamma_{a}, \gamma_{b}, \gamma_{c}\right)$ taken from Table $I$ and $Q$ is an isotropically distributed unitary matrix independent of $\eta$.

Table I

GDOF OF THE $2 \times 2$ MIMO CHANNEL WITH $\gamma_{11}=\gamma_{22}=\gamma_{D} \geq \gamma_{C L}=\gamma_{12}=\gamma_{21}$

\begin{tabular}{|l|l|l|}
\hline Regime & Solution $\left(\gamma_{a}, \gamma_{b}, \gamma_{c}\right)$ & $\mathrm{gDoF}$ \\
\hline \hline$T=2$ & $\left(0,0, \gamma_{C L}\right)$ & $\gamma_{D}-\frac{1}{2} \gamma_{C L}$ \\
\hline$T \geq 3$ & $(0,0,0)$ & $2\left(\left(1-\frac{1}{T}\right) \gamma_{D}-\frac{1}{T} \gamma_{C L}\right)$ \\
\hline
\end{tabular}

Proof idea: From Theorem 1, we have an optimal distribution of the form

$$
X=\left[\begin{array}{cccccc}
a & 0 & 0 & . & . & 0 \\
b & c & 0 & . & . & 0
\end{array}\right] Q
$$

where $Q$ is an isotropically distributed unitary matrix independent of $a, b, c$. We first obtain a capacity outer bound as the maximum of the expected value of a function $f\left(|a|^{2},|b|^{2},|c|^{2}\right)$. This is using Lemma 19. Lemma 20 and Lemma 21 which help to convert the entropy terms $h()$ into expected values. Then, in Lemma 22 we prove that the maximization of $\mathbb{E}\left[f\left(|a|^{2},|b|^{2},|c|^{2}\right)\right]$ can have a solution with a single mass point of $\left(|a|^{2},|b|^{2},|c|^{2}\right)$ for achieving the gDoF. Then the $\mathrm{gDoF}$ outer bound can be expressed as the solution of a piecewise linear optimization problem, which yields the solution as above. The detailed proof of the outer bound is in Section IV-D. The inner bound can be verified by using the distribution stated in the Theorem to evaluate the mutual information; the calculation is given in Appendix D. Also note that $\gamma_{D} \geq \gamma_{C L}$ is without loss of generality, since we can relabel the links to satisfy this condition. 
Note that the above result shows that we need to use both the antennas for achieving the gDoF for $T=2$, since with only one antenna we can only achieve $(1 / 2) \gamma_{D}$ due to Theorem 4. This is in contrast to the result for the $2 \times 2 \mathrm{MIMO}$ channel with i.i.d. links, where the $\mathrm{gDoF}$ could be achieved using a single transmit antenna for $T=2$; also, using both the antennas was sub-optimal [3]. For $T \geq 3$, for a $2 \times 2$ MIMO channel with the value $\gamma_{D}$ for SNR exponents in all the links, the gDoF is $2(1-2 / T) \gamma_{D}$ [3], whereas in our model with direct link exponents $\gamma_{D}$ and cross link exponents $\gamma_{C L}$, the $\mathrm{gDoF}$ is $2\left((1-1 / T) \gamma_{D}-(1 / T) \gamma_{C L}\right)$. Thus having weaker crosslinks gives a gDoF gain of $(2 / T)\left(\gamma_{D}-\gamma_{C L}\right)$. Also as $T \rightarrow \infty$, the gDoF achieved is $2 \gamma_{D}$, which agrees with the gDoF results for the coherent MIMO channel [20, Theorem 2].

Also, it is clear that training-based schemes are suboptimal for the $2 \times 2$ MIMO channel. For $T=2$, if one were to train the links, one has to use two time slots, which leaves no time for communicating. For $T=2$, if one were to use only one transmit antenna and use one training slot, the gDoF achievable is $\gamma_{D} / 2$ which is smaller than what we achieve. For $T \geq 3$, the gDoF achievable after using two time slots to communicate is $2(1-2 / T) \gamma_{D}$ which is less than the gDoF $2\left((1-1 / T) \gamma_{D}-(1 / T) \gamma_{C L}\right)$ that we achieve. The gain in gDoF that we have is $(2 / T)\left(\gamma_{D}-\gamma_{C L}\right)$.

Table II

COMPARISON OF RATES ACHIEVABLE FOR THE $2 \times 2$ MIMO CHANNEL WITH DIFFERENT SCHEMES FOR $T=2$,

$$
\mathbb{E}\left[\left|g_{11}\right|^{2}\right]=\mathbb{E}\left[\left|g_{22}\right|^{2}\right]=0.1
$$

\begin{tabular}{|c|c|c|c|c|c|}
\hline \multirow{2}{*}{ Transmit SNR per antenna $(\mathrm{dB})$} & \multirow{2}{*}{$\mathbb{E}\left[\left|g_{21}\right|^{2}\right]$} & \multicolumn{2}{|c|}{ Rates for different schemes } & \multirow{2}{*}{ Gain using noncoherent scheme } \\
\cline { 3 - 5 } & & Noncoherent & SISO & Parallel & 0.059 \\
\hline 22 & .025 & 1.364 & 1.305 & 1.063 & 0.098 \\
\hline 23 & .025 & 1.536 & 1.438 & 1.095 & 0.220 \\
\hline 23 & .016 & 1.657 & 1.438 & 1.396 & 0.017 \\
\hline 23 & .040 & 1.454 & 1.438 & 0.807 & \\
\hline
\end{tabular}

Although Theorem 7 is for the $\mathrm{gDoF}$ of the system, our results can provide design guidelines for specific scenarios. For example, for a $2 \times 2$ system with coherence time $T=2$, transmit $\mathrm{SNR}=23 \mathrm{~dB}$, direct links with average strength 0.1 and crosslinks with average strength 0.025 (which corresponds to $\gamma_{D}=0.56, \gamma_{C L}=0.30$ ), our noncoherent scheme can obtain a $7 \%$ gain in the rate compared to the schemes that use a symbol for training. We illustrate more 
examples ${ }^{3}$ in Table II where our noncoherent scheme can obtain gain in the rates compared to the schemes that use a symbol for training. One possible training-based scheme is to use only one antenna (reducing the MIMO channel to a SISO channel) and using one symbol to train the channel. Another possible scheme is to use both antennas and treat the system as a parallel antenna system, treating the crosslinks as noise. For the parallel case also, the training-based scheme uses one symbol to train the channel. The rate points in Table $\Pi$ are just a few examples of some specific scenarios, but we believe that there would be many other cases where this approach is useful when we have short coherence time and asymmetry in channel gains. The details of the expressions used for the numerics are given in Appendix J.

\section{Nonoptimality of Training}

We observed in the previous subsection that training-based schemes cannot achieve the $\mathrm{gDoF}$ for $2 \times 2$ MIMO channels in general. We can extend this observation to larger MIMO channels. We specifically consider the $M \times M$ MIMO channel with exponents $\gamma_{D}$ in the direct links and $\gamma_{C L}$ in the crosslinks $\left(\gamma_{D}>\gamma_{C L}\right)$. Using the following theorem, we prove that training-based schemes are suboptimal for this case.

Theorem 8. $A$ gDoF of $M\left((1-1 / T) \gamma_{D}-((M-1) / T) \gamma_{C L}\right)$ can be achieved for an $M \times M$ MIMO channel with coherence time $T>M$ and with exponents $\gamma_{D}$ in the direct links and $\gamma_{C L}$ in the crosslinks $\left(\gamma_{D}>\gamma_{C L}\right)$ (Figure 8), by using i.i.d. Gaussian codebooks across the antennas and time periods.

Proof: In this case, the channel matrix $G$ has diagonal elements $g_{i i}$ distributed according to $\mathcal{C N}\left(0, \mathrm{SNR}^{\gamma_{D}}\right)$ and the rest of the elements are distributed according to $\mathcal{C N}\left(0, \mathrm{SNR}^{\gamma_{C L}}\right)$. Using Gaussian codebooks, the rate $R \geq I(G X+W ; X)$ is achievable with $X$ being an $M \times T$ matrix with i.i.d. $\mathcal{C N}(0,1)$ elements. Analyzing this mutual information yields an achievable gDoF of $M\left((1-1 / T) \gamma_{D}-((M-1) / T) \gamma_{C L}\right)$ per symbol. The calculations are given in Appendix $\mathrm{E}$

Note that the $\mathrm{gDoF} M\left((1-1 / T) \gamma_{D}-((M-1) / T) \gamma_{C L}\right)$ cannot be achieved by a conventional training scheme where all transmitters train independently. This is clear since it requires $M$ symbols in every coherence period for training and the maximum gDoF achievable using the rest

\footnotetext{
${ }^{3}$ The link strengths in the examples are given without absorbing the transmit SNR into them.
} 


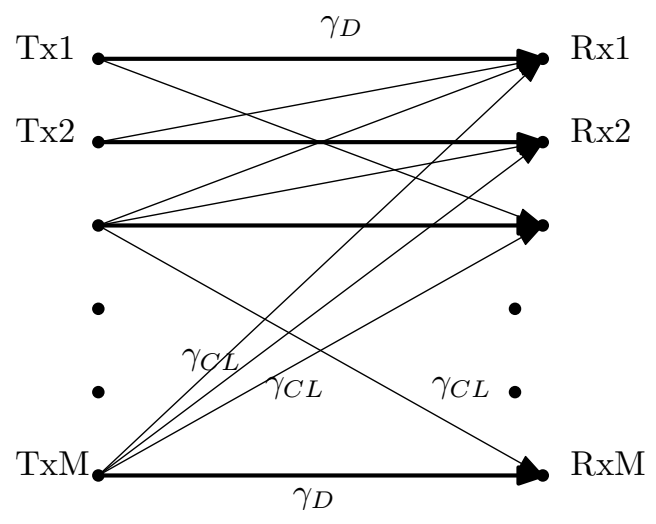

Figure 8. The $M \times M$ MIMO channel with exponents $\gamma_{D}$ in the direct links and $\gamma_{C L}$ in crosslinks.

of the symbols is $M(1-M / T) \gamma_{D}$ [20, Theorem 2], assuming that the channels are available perfectly due to training. This is smaller than $M\left((1-1 / T) \gamma_{D}-((M-1) / T) \gamma_{C L}\right)$. Thus using Gaussian codebooks and not using training give a gDoF gain of $(M(M-1) / T)\left(\gamma_{D}-\gamma_{C L}\right)$. This result also suggests that for noncoherent networks with multiple nodes, a training-based scheme may not be gDoF-optimal, by viewing the cut-sets of the networks as MIMO channels.

\section{ANALYSIS}

In this section, we provide a more detailed analysis for the results stated in the previous section. We first state some mathematical preliminaries required for the analysis. Then in Section IV-B, we derive the structure of the capacity achieving distribution for the noncoherent MIMO channel. In Section IV-C, we prove the gDoF outer bounds for the noncoherent MISO channel and in Section IV-D, we derive the $\mathrm{gDoF}$ outer bounds for the $2 \times 2$ MIMO system.

\section{A. Mathematical Preliminaries}

Lemma 9. For an exponentially distributed random variable $\xi$ with mean $\mu_{\xi}$ and for given constants $a \geq 0, b>0$,

$$
\log \left(a+b \mu_{\xi}\right)-\gamma_{E} \log (e) \leq \mathbb{E}[\log (a+b \xi)] \leq \log \left(a+b \mu_{\xi}\right)
$$

where $\gamma_{E}$ is Euler's constant.

Proof: This is given in [21, Section VI-B]. 
Lemma 10. For a chi-squared random variable $\chi^{2}(k)$ and for given constants $a \geq 0, b>0$,

$$
\log (a+b k)-\frac{\log (e) 2}{k}+\log \left(1+\frac{1}{k}\right) \leq \mathbb{E}\left[\log \left(a+b \chi^{2}(k)\right)\right] \leq \log (a+b k) .
$$

Proof: The result is proved in [21, Section VI-A] for the Gamma distribution and the result for the chi-squared distribution follows as a special case.

Lemma 11. For an exponential random variable $\xi$ with mean $\mu_{\xi}$ and for a given constant $b>0$, we have

$$
\mathbb{E}\left[\frac{b}{b+\xi}\right]=\frac{b}{\mu_{\xi}} e^{\frac{b}{\mu_{\xi}}} E_{1}\left(\frac{b}{\mu_{\xi}}\right)
$$

and

$$
1>\frac{b}{\mu_{\xi}} \ln \left(1+\frac{\mu_{\xi}}{b}\right) \geq \frac{b}{\mu_{\xi}} e^{\frac{b}{\mu_{\xi}}} E_{1}\left(\frac{b}{\mu_{\xi}}\right) \geq \frac{b}{2 \mu_{\xi}} \ln \left(1+\frac{2 \mu_{\xi}}{b}\right),
$$

where $E_{1}(\cdot)$ is the exponential integral function.

Proof: We have

$$
\begin{aligned}
\mathbb{E}\left[\frac{b}{b+\xi}\right] & =\mathbb{E}\left[\frac{1}{1+\frac{\xi}{b}}\right] \\
& \stackrel{(i)}{=} \int_{0}^{\infty} \frac{b}{\mu_{\xi}} e^{-\frac{b x}{\mu_{\xi}}} \frac{1}{1+x} d x \\
& \stackrel{(i i))}{=} \frac{b}{\mu_{\xi}} e^{\frac{b}{\mu_{\xi}}} \int_{1}^{\infty} e^{-\frac{b x}{\mu_{\xi}}} \frac{1}{x} d x \\
& \stackrel{(i i i)}{=} \frac{b}{\mu_{\xi}} e^{\frac{b}{\mu_{\xi}}} \int_{\frac{b}{\mu_{\xi}}}^{\infty} e^{-t} \frac{1}{t} d t \\
& \stackrel{(i v)}{=} \frac{b}{\mu_{\xi}} e^{\frac{b}{\mu_{\xi}}} E_{1}\left(\frac{b}{\mu_{\xi}}\right) .
\end{aligned}
$$

where the step $(i)$ is because $\xi / b$ is exponentially distributed with mean $\mu_{\xi} / b$, the steps $(i i)$, $(i i i)$ are by change of variables, and the step $(i v)$ is by the definition of the exponential integral. We have

$$
\frac{1}{2} \ln \left(1+\frac{1}{x}\right) \leq e^{x} E_{1}(x) \leq \ln \left(1+\frac{1}{x}\right)
$$

from [22]. Also $\left(b / \mu_{\xi}\right) \ln \left(1+\mu_{\xi} / b\right)<1$, because $0<x \ln (1+1 / x)<1$ for $x>0$. Thus the desired result follows.

Lemma 12. Let $H$ be an isotropically distributed random matrix and $\Phi$ be a random unitary matrix distributed according to any distribution independent of $H$, then $H, \Phi H, H \Phi$ all have the same distribution. Moreover, $\Phi H$ and $H \Phi$ are independent of $\Phi$. See [2] for details. 
Lemma 13. Let $\left[\xi_{1}, \xi_{2}, \ldots, \xi_{n}\right]$ be an arbitrary complex random vector and $Q$ be an $n \times n$ isotropically distributed random unitary matrix independent of $\xi_{i}$, then

$$
h\left(\left[\xi_{1}, \xi_{2}, \ldots, \xi_{n}\right] Q\right)=h\left(\sum\left|\xi_{i}\right|^{2}\right)+(n-1) \mathbb{E}\left[\log \left(\sum\left|\xi_{i}\right|^{2}\right)\right]+\log \left(\frac{\pi^{n}}{\Gamma(n)}\right) .
$$

Proof idea: This is proved by using the fact that in radial coordinates, the distribution of $\left[\xi_{1}, \xi_{2}, \ldots, \xi_{n}\right] Q$ is dependent only on the radius. See Appendix $\mathrm{A}$ for more details.

Note that we can use the above Lemma also on $h\left(\xi_{1} \bar{q}_{1}{ }^{(T)}\right)$ with an isotropically distributed random unit vector ${\overline{q_{1}}}^{(T)}$ by considering the equality $h\left(\xi_{1}{\overline{q_{1}}}^{(T)}\right)=h\left(\left[\xi_{1}, 0, \ldots, 0\right] Q\right)$, where the isotropically distributed random unit vector ${\overline{q_{1}}}^{(T)}$ can be taken as the first row of an isotropically distributed random unitary matrix $Q$.

Corollary 14. Let $\left[\xi_{1}, \xi_{2}, \ldots, \xi_{n}\right]$ be an arbitrary complex random vector, $\xi$ be an arbitrary complex random variable and $Q$ be an $n \times n$ isotropically distributed random unitary matrix independent of $\xi, \xi_{i}$, then

$$
h\left(\left[\xi_{1}, \xi_{2}, \ldots, \xi_{n}\right] Q \mid \xi\right)=h\left(\sum\left|\xi_{i}\right|^{2} \mid \xi\right)+(n-1) \mathbb{E}\left[\log \left(\sum\left|\xi_{i}\right|^{2}\right)\right]+\log \left(\frac{\pi^{n}}{\Gamma(n)}\right) .
$$

Proof: This can be proved similar to the previous lemma since the distribution of $h\left(\left[\xi_{1}, \xi_{2}, \ldots, \xi_{n}\right] Q \mid \xi\right)$ will be dependent only on the radius.

We can use this corollary also on $h\left(\xi_{1}{\overline{q_{1}}}^{(T)} \mid \xi\right)$, similar to the previous Lemma.

Lemma 15. Among positive continuous random variables with a given mean, the exponential random variable has the maximum entropy.

Proof: See [23, Chapter 12].

\section{B. Properties of Transmitted Signals that Achieve Capacity}

We now establish the properties of capacity achieving distribution for the noncoherent MIMO channel with asymmetric statistics. We have our channel model $Y=G X+W$. Now for any $T \times T$ unitary matrix $\Phi$ we have $Y \Phi^{\dagger}=G X \Phi^{\dagger}+W \Phi^{\dagger}$. Since $w_{i j}$ are i.i.d. $\mathcal{C N}(0,1), W \Phi^{\dagger}$ and $W$ have the same distribution, and hence

$$
p\left(Y \Phi^{\dagger} \mid X \Phi^{\dagger}\right)=p(Y \mid X)
$$


Now

$$
C=\sup _{p(X)} I(X ; Y)
$$

subject to the average power constraint (4) and we have

$$
\begin{aligned}
I(X ; Y) & =\mathbb{E}\left[\log \left(\frac{p(Y \mid X)}{p(Y)}\right)\right] \\
& =\int d X p(X) \int d Y p(Y \mid X) \log \left(\frac{p(Y \mid X)}{\int d \tilde{X} p(\tilde{X}) p(Y \mid \tilde{X})}\right) .
\end{aligned}
$$

Lemma 16. (Invariance of $I(X ; Y)$ to post-rotations of $X)$ : Suppose that $X$ has a probability density $p_{0}(X)$ that generates some mutual information $I_{0}$. Then, for any unitary matrix $\Phi$, the “post-rotated” probability density, $p_{1}(X)=p_{0}\left(X \Phi^{\dagger}\right)$ also generates $I_{0}$.

Proof idea: This is an adaptation of the existing results for MIMO channels from [2, Lemma 1]. The proof proceeds by substituting the post-rotated density $p_{1}(X)$ into (17), changing the variables of integration and using $p(Y \Phi \mid X \Phi)=p(Y \mid X)$ from $(15)$.

Lemma 17. The signal of the form $X=L Q$ with $L$ being a lower triangular random matrix and $Q$ being an isotropically distributed random unitary matrix independent of $L$ achieves the capacity of the noncoherent MIMO channel.

Proof: Let $X$ be a capacity achieving random variable and $I_{0}$ be the corresponding mutual information achieved. Now $X$ can be decomposed as $X=L \Phi^{\prime}$ using the LQ decomposition with $L$ upper diagonal and $\Phi^{\prime}$ unitary, but they could be jointly distributed and $\Phi^{\prime}$ may not be isotropically unitary distributed. Let $\Theta$ be an isotropically distributed random unitary matrix that is independent of $L$ and $\Phi^{\prime}$. Now use $X_{1}=X \Theta$ for signaling. Let $Y$ be the corresponding received signal. Then

$$
\begin{aligned}
I\left(X_{1} ; Y \mid \Theta\right) & =I(X \Theta ; Y \mid \Theta) \\
& =I_{0}
\end{aligned}
$$

where the last step was using Lemma 16. Now

$$
\begin{array}{r}
I\left(X_{1} ; Y\right)+I\left(\Theta ; Y \mid X_{1}\right)=I(\Theta ; Y)+I\left(X_{1} ; Y \mid \Theta\right) \\
I\left(X_{1} ; Y\right)+0 \stackrel{(i)}{=} I(\Theta ; Y)+I\left(X_{1} ; Y \mid \Theta\right) \\
I\left(X_{1} ; Y\right) \stackrel{(i i)}{\geq} I\left(X_{1} ; Y \mid \Theta\right)
\end{array}
$$




$$
=I_{0}
$$

where $(i)$ was because $I\left(\Theta ; Y \mid X_{1}\right)=0$ since $\Theta-X_{1}-Y$ is a Markov chain and $(i i)$ was because $I\left(X_{1} ; Y \mid \Theta\right) \geq 0$. Hence without loss of generality, the signal of the form $L Q=L \Phi^{\prime} \Theta$ with $Q=\Phi^{\prime} \Theta$ achieves the capacity. Now $Q=\Phi^{\prime} \Theta$ is an isotropically distributed unitary matrix and it is independent of $\Phi^{\prime}$ using Lemma 12 on page 17 .

Next, we focus our attention on computing $h(Y \mid X)$, which will be necessary in future derivations. Let $Y(n)$ be the $n^{\text {th }}$ row of $Y$. Conditioned on $X$, the rows of $Y$ are independent Gaussian. Hence

$$
h(Y \mid X)=\sum_{n=1}^{N} h(Y(n) \mid X) .
$$

With $\underline{\rho}^{2}(n)$ being the vector of channel strengths arriving at the $n^{\text {th }}$ receiver antenna, we have:

$$
\begin{aligned}
K_{Y(n) \mid X} & =\mathbb{E}\left[Q^{\dagger} L^{\dagger} g^{\dagger}(n) g(n) L Q \mid L Q\right]+I_{T} \\
& =Q^{\dagger} L^{\dagger} \mathbb{E}\left[g^{\dagger}(n) g(n)\right] L Q+I_{T} \\
& =Q^{\dagger} L^{\dagger} \operatorname{diag}\left(\underline{\rho}^{2}(n)\right) L Q+I_{T},
\end{aligned}
$$

where $I_{T}$ is a $T \times T$ identity matrix and $\operatorname{diag}\left(\underline{\rho}^{2}(n)\right)$ is the diagonal matrix formed from $\underline{\rho}^{2}(n)$. Hence

$$
\begin{aligned}
h(Y(n) \mid X) \\
\quad=\mathbb{E}\left[\log \left(\operatorname{det}\left(\pi e K_{Y(n) \mid X}\right)\right)\right] \\
\quad=\mathbb{E}\left[\log \left(\operatorname{det}\left(\pi e\left(Q^{\dagger} L^{\dagger} \operatorname{diag}\left(\underline{\rho}^{2}(n)\right) L Q+I_{T}\right)\right)\right)\right] \\
\quad \stackrel{(i)}{=} \mathbb{E}\left[\log \left(\operatorname{det}\left(\pi e\left(L^{\dagger} \operatorname{diag}\left(\underline{\rho}^{2}(n)\right) L+I_{T}\right)\right)\right)\right],
\end{aligned}
$$

where $(i)$ uses the property of determinants to cancel $Q$ and $Q^{\dagger}$. Also, for $T \geq M$, with $L_{M \times M}$ being the first $M \times M$ submatrix of $L$ (rest of the elements of $L$ are zero for $T \geq M$ ), we have:

$$
\begin{aligned}
& h(Y(n) \mid X) \\
& =\mathbb{E}\left[\log \left(\operatorname{det}\left(L_{M \times M}^{\dagger} \operatorname{diag}\left(\underline{\rho}^{2}(n)\right) L_{M \times M}+I_{M}\right)\right)\right]+T \log (\pi e) .
\end{aligned}
$$

\section{Outer Bound for the $M \times 1$ MISO Channel}

We now prove the gDoF outer bound given in Theorem 6 for the $M \times 1$ MISO system. We assume that $T>1$, since for $T=1$ we have the desired result using Theorem 5 on page 11 
Also, we assume that $T \geq M$ in the following outer bound computations. The case for $T<M$ can be derived similarly and the derivation is given in Appendix $\mathrm{F}$,

For the capacity achieving distribution, we have the structure $X=\left[\begin{array}{ll}L_{M \times M} & 0_{M \times(T-M)}\end{array}\right] Q$ (from Theorem 11), where

$$
L_{M \times M}=\left[\begin{array}{cccc}
x_{11} & 0 & 0 & \\
\cdot & \cdot & 0 & 0 \\
\cdot & & \cdot & 0 \\
x_{M 1} & \cdot & \cdot & x_{M M}
\end{array}\right],
$$

and $0_{M \times(T-M)}$ is an $M \times(T-M)$ matrix with elements of value zero. Also $Q$ is an isotropically distributed random unitary matrix. For the MISO channel, we have $Y=G X+W$ with $G=$ $\left[\begin{array}{llll}g_{11} & . & g_{1 M}\end{array}\right]$ and $W$ is the $1 \times T$ noise vector with i.i.d. $\mathcal{C N}(0,1)$ components. We assume $\rho_{11}^{2} \geq \rho_{1 i}^{2}$ without loss of generality. Now note that $W Q$ has also the same distribution as $W$ and is independent of $Q$ (using the fact that $W$ is isotropically distributed and Lemma 12). Hence

$$
\begin{aligned}
& h(Y)=h((G X+W) Q) \\
& =h\left(\left[\left(w_{11}+\sum_{i=1}^{M} x_{i 1} g_{1 i}\right), \quad\left(w_{12}+\sum_{i=2}^{M} x_{i 2} g_{1 i}\right), \ldots\right.\right. \\
& \left.\left.\ldots,\left(w_{1 M}+\sum_{i=M}^{M} x_{i 2} g_{1 i}\right), \quad w_{1(M+1)} \ldots, w_{1 T}\right] Q\right),
\end{aligned}
$$

where $w_{1 i}$ are i.i.d. and $\mathcal{C N}(0,1)$ distributed. Now using Lemma 13 on page 18 , we have

$$
\begin{aligned}
& h(Y)=h\left(\sum_{j=1}^{M}\left|w_{1 j}+\sum_{i=j}^{M} x_{i j} g_{1 i}\right|^{2}+\sum_{i=M+1}^{T}\left|w_{1 i}\right|^{2}\right) \\
&+(T-1) \mathbb{E}\left[\log \left(\sum_{j=1}^{M}\left|w_{1 j}+\sum_{i=j}^{M} x_{i j} g_{1 i}\right|^{2}+\sum_{i=M+1}^{T}\left|w_{1 i}\right|^{2}\right)\right]+\log \left(\frac{\pi^{T}}{\Gamma(T)}\right) \\
& \stackrel{(i)}{\leq} h\left(\sum_{j=1}^{M}\left|w_{1 j}+\sum_{i=j}^{M} x_{i j} g_{1 i}\right|^{2}+\sum_{i=M+1}^{T}\left|w_{1 i}\right|^{2}\right) \\
&+(T-1) \mathbb{E}\left[\log \left(\sum_{i=1}^{M} \rho_{1 i}^{2}\left(\sum_{j=1}^{i}\left|x_{i j}\right|^{2}\right)+T-M\right)\right]+\log \left(\frac{\pi^{T}}{\Gamma(T)}\right),
\end{aligned}
$$

where $(i)$ is using the Tower property of expectation, Jensen's inequality and $\sum_{j=1}^{M} \sum_{i=j}^{M}\left|x_{i j}\right|^{2} \rho_{1 i}^{2}=$ $\sum_{i=1}^{M} \sum_{j=1}^{i}\left|x_{i j}\right|^{2} \rho_{1 i}^{2}$. Now using 28 we have

$$
h(Y \mid X)=\mathbb{E}\left[\log \left(\operatorname{det}\left(L_{M \times M}^{\dagger} \operatorname{diag}\left(\rho_{11}^{2}, \ldots, \rho_{1 M}^{2}\right) L_{M \times M}+I_{M}\right)\right)\right]
$$




$$
\begin{aligned}
&+T \log (\pi e) \\
&=\mathbb{E}\left[\log \left(\prod_{i=1}^{M}\left(1+\omega_{i}\right)\right)\right]+T \log (\pi e),
\end{aligned}
$$

where $\omega_{i}$ are the eigenvalues of $L_{M \times M}^{\dagger} \operatorname{diag}\left(\rho_{11}^{2}, \ldots, \rho_{1 M}^{2}\right) L_{M \times M}$. The eigenvalues are nonnegative since the matrix is Hermitian. Hence

$$
\begin{aligned}
h(Y \mid X) & =\mathbb{E}\left[\log \left(\prod_{i=1}^{M}\left(1+\omega_{i}\right)\right)\right]+T \log (\pi e) \\
& \geq \mathbb{E}\left[\log \left(1+\sum \omega_{i}\right)\right]+T \log (\pi e) .
\end{aligned}
$$

The last step is true because $\omega_{i} \geq 0$. Now

$$
\begin{aligned}
\sum \omega_{i} & =\operatorname{Trace}\left(L_{M \times M}^{\dagger} \operatorname{diag}\left(\rho_{11}^{2}, \ldots, \rho_{1 M}^{2}\right) L_{M \times M}\right) \\
& =\operatorname{Trace}\left(\operatorname{diag}\left(\rho_{11}^{2}, \ldots, \rho_{1 M}^{2}\right) L_{M \times M} L_{M \times M}^{\dagger}\right) \\
& =\sum_{i=1}^{M} \rho_{1 i}^{2}\left(\sum_{j=1}^{i}\left|x_{i j}\right|^{2}\right) .
\end{aligned}
$$

Hence

$$
h(Y \mid X) \geq \mathbb{E}\left[\log \left(1+\sum_{i=1}^{M} \rho_{1 i}^{2}\left(\sum_{j=1}^{i}\left|x_{i j}\right|^{2}\right)\right)\right]+T \log (\pi e)
$$

Hence

$$
\begin{aligned}
I(X ; Y) \leq h & \left(\sum_{j=1}^{M}\left|w_{1 j}+\sum_{i=j}^{M} x_{i j} g_{1 i}\right|^{2}+\sum_{i=M+1}^{T}\left|w_{1 i}\right|^{2}\right) \\
& +(T-1) \mathbb{E}\left[\log \left(\sum_{i=1}^{M} \rho_{1 i}^{2}\left(\sum_{j=1}^{i}\left|x_{i j}\right|^{2}\right)+T-M\right)\right] \\
& -\mathbb{E}\left[\log \left(1+\sum_{i=1}^{M} \rho_{1 i}^{2}\left(\sum_{j=1}^{i}\left|x_{i j}\right|^{2}\right)\right)\right] \\
& +\log \left(\frac{\pi^{T}}{\Gamma(T)}\right)-T \log (\pi e) \\
\leq( & -1) \log \left(\sum_{i=1}^{M} \rho_{1 i}^{2} M T+T\right)
\end{aligned}
$$

where in the last step, we used Lemma 15 and Jensen's inequality. Hence

$$
\limsup _{\text {SNR } \rightarrow \infty} \frac{1}{T} \frac{I(X ; Y)}{\log (\mathrm{SNR})} \leq\left(1-\frac{1}{T}\right) \gamma_{11} \text {. }
$$




\section{Outer Bound for the $2 \times 2$ MIMO Channel}

In this subsection, we prove the gDoF outer bound from Theorem 7 for the $2 \times 2$ MIMO channel with exponents $\gamma_{D}$ in the direct links and $\gamma_{C L}$ in the crosslinks. We have the structure of the optimal distribution as

$$
X=\left[\begin{array}{llllll}
a & 0 & 0 & . & 0 \\
b & c & 0 & . & . & 0
\end{array}\right] Q
$$

from Theorem 11, We have

$$
G=\left[\begin{array}{ll}
g_{11} & g_{12} \\
g_{21} & g_{22}
\end{array}\right],
$$

and $Y=G X+W$, where $W$ is a $2 \times T$ vector with i.i.d. $\mathcal{C N}(0,1)$ components. For $T=1$, the $\mathrm{gDoF}$ is zero due to Theorem 5 , hence we consider $T \geq 2$ in this proof. We have

$$
\begin{aligned}
& h(Y)=h\left(G\left[\begin{array}{llllll}
a & 0 & 0 & \cdot & \cdot & 0 \\
b & c & 0 & . & \cdot & 0
\end{array}\right] Q+W\right)
\end{aligned}
$$

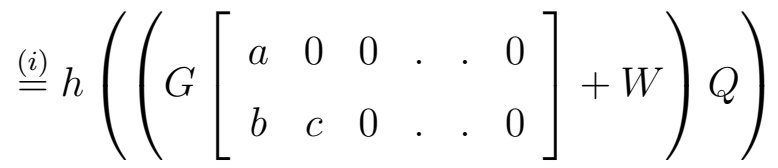

$$
\begin{aligned}
& =h\left(\left[\begin{array}{ccccc}
a g_{11}+b g_{12}+w_{11} & c g_{12}+w_{12} & w_{13} & \cdot w_{1 T} \\
a g_{21}+b g_{22}+w_{21} & c g_{22}+w_{22} & w_{23} & \cdot & w_{2 T}
\end{array}\right] Q\right)
\end{aligned}
$$

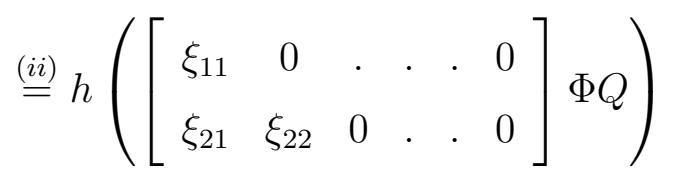

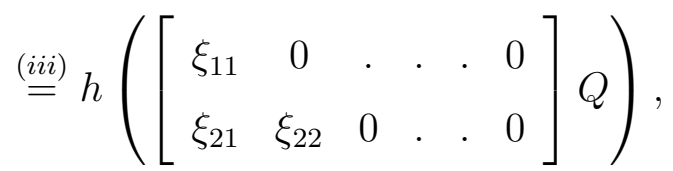

where the step $(i)$ used the fact that $W$ and $W Q$ have the same distribution and $W Q$ is independent of $Q$. In step $(i i), \xi_{i j}$ arise after LQ transformation (using Gram-Schmidt process):

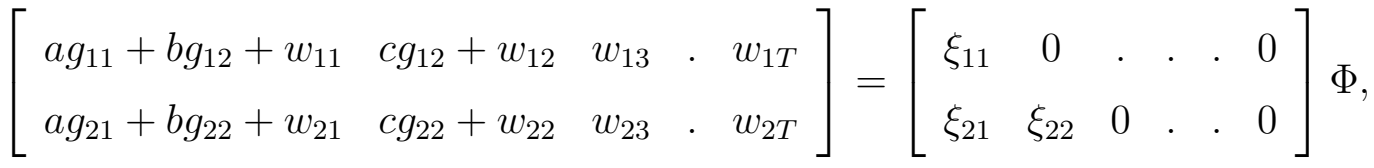

$$
\begin{aligned}
& \left|\xi_{11}\right|^{2}=\left|a g_{11}+b g_{12}+w_{11}\right|^{2}+\left|c g_{12}+w_{12}\right|^{2}+\sum_{i=3}^{T}\left|w_{1 i}\right|^{2},
\end{aligned}
$$




$$
\begin{aligned}
\left|\xi_{21}\right|^{2}= & \frac{\left|\left(a g_{21}+b g_{22}+w_{21}\right)\left(a g_{11}+b g_{12}+w_{11}\right)^{*}+\left(c g_{22}+w_{22}\right)\left(c g_{12}+w_{12}\right)^{*}+\sum_{i=3}^{T} w_{2 i} w_{1 i}^{*}\right|^{2}}{\left|a g_{11}+b g_{12}+w_{11}\right|^{2}+\left|c g_{12}+w_{12}\right|^{2}+\sum_{i=3}^{T}\left|w_{1 i}\right|^{2}} \\
\left|\xi_{22}\right|^{2}= & \left|a g_{21}+b g_{22}+w_{21}\right|^{2}+\left|c g_{22}+w_{22}\right|^{2}+\sum_{i=3}^{T}\left|w_{2 i}\right|^{2} \\
& -\frac{\left|\left(a g_{21}+b g_{22}+w_{21}\right)\left(a g_{11}+b g_{12}+w_{11}\right)^{*}+\left(c g_{22}+w_{22}\right)\left(c g_{12}+w_{12}\right)^{*}+\sum_{i=3}^{T} w_{2 i} w_{1 i}^{*}\right|^{2}}{\left|a g_{11}+b g_{12}+w_{11}\right|^{2}+\left|c g_{12}+w_{12}\right|^{2}+\sum_{i=3}^{T}\left|w_{1 i}\right|^{2}}
\end{aligned}
$$

where $\Phi$ is unitary. In step $(i i i)$, we absorb $\Phi$ onto $Q$ using Lemma 12, The Gram-Schmidt process for LQ transformation yields $\xi_{i j}$ as given in (47), (48) and (49).

Also, using (28), (24), we get

$$
\begin{aligned}
h(Y \mid X)= & \mathbb{E}\left[\log \left(|a|^{2} \rho_{11}^{2}+|b|^{2} \rho_{12}^{2}+|c|^{2} \rho_{12}^{2}+|a|^{2}|c|^{2} \rho_{11}^{2} \rho_{12}^{2}+1\right)\right] \\
& +\mathbb{E}\left[\log \left(|a|^{2} \rho_{21}^{2}+|b|^{2} \rho_{22}^{2}+|c|^{2} \rho_{22}^{2}+|a|^{2}|c|^{2} \rho_{21}^{2} \rho_{22}^{2}+1\right)\right] \\
& +2 T \log (\pi e) .
\end{aligned}
$$

For computing $h(Y)$, let $\bar{q}_{1}^{(T)}, \bar{q}_{2}^{(T)}$ be the first two rows of $Q$. The vectors $\bar{q}_{1}^{(T)}, \bar{q}_{2}^{(T)}$ are orthogonal since $Q$ is unitary. We have

$$
\begin{aligned}
& h(Y)=h\left(\left[\begin{array}{cccccc}
\xi_{11} & 0 & . & . & 0 \\
\xi_{21} & \xi_{22} & 0 & . & . & 0
\end{array}\right] Q\right) \\
& =h\left(\xi_{11} \bar{q}_{1}^{(T)}\right)+h\left(\xi_{21} \bar{q}_{1}^{(T)}+\xi_{22} \bar{q}_{2}^{(T)} \mid \xi_{11} \bar{q}_{1}^{(T)}\right) \text {. }
\end{aligned}
$$

Now consider $h\left(\xi_{21} \bar{q}_{1}^{(T)}+\xi_{22} \bar{q}_{2}^{(T)} \mid \xi_{11} \bar{q}_{1}^{(T)}\right)$. Since $\xi_{11}$ is nonnegative and $\xi_{11} \bar{q}_{1}^{(T)}$ is given in the conditioning, the direction $\bar{q}_{1}^{(T)}$ is known in the conditioning. Hence considering $\xi_{21} \bar{q}_{1}^{(T)}+\xi_{22} \bar{q}_{2}^{(T)}$ in a new orthonormal basis with the first basis vector chosen as $\bar{q}_{1}^{(T)}$ and the rest of the basis vectors chosen arbitrarily, the projection of $\xi_{21} \bar{q}_{1}^{(T)}+\xi_{22} \bar{q}_{2}^{(T)}$ onto the first basis vector is $\xi_{21}$. The projection onto the rest of the $T-1$ vectors forms $\xi_{22} \bar{q}_{2}^{(T-1)}$ where $\bar{q}_{2}^{(T-1)}$ is a $T-1$ dimensional isotropically distributed random unit vector. Hence

$$
\begin{aligned}
h\left(\xi_{21} \bar{q}_{1}^{(T)}+\xi_{22} \bar{q}_{2}^{(T)} \mid \xi_{11} \bar{q}_{1}^{(T)}\right) & =h\left(\left[\xi_{21}, \xi_{22} \bar{q}_{2}^{(T-1)}\right] \mid \xi_{11}, \bar{q}_{1}^{(T)}\right) \\
& =h\left(\left[\xi_{21}, \xi_{22} \bar{q}_{2}^{(T-1)}\right] \mid \xi_{11}\right)
\end{aligned}
$$


and

$$
\begin{aligned}
h(Y) & =h\left(\xi_{11} \bar{q}_{1}^{(T)}\right)+h\left(\left[\xi_{21}, \xi_{22} \bar{q}_{2}^{(T-1)}\right] \mid \xi_{11}\right) \\
& \stackrel{(i)}{=} h\left(\xi_{11} \bar{q}_{1}^{(T)}\right)+h\left(\left.\left[\xi_{21}, \xi_{22} \bar{q}_{2}^{(T-1)}\right]|| \xi_{11}\right|^{2}\right) \\
& \leq h\left(\xi_{11} \bar{q}_{1}^{(T)}\right)+h\left(\left.\xi_{22} \bar{q}_{2}^{(T-1)}|| \xi_{11}\right|^{2}\right)+h\left(\left.\xi_{21}|| \xi_{11}\right|^{2}\right),
\end{aligned}
$$

where $(i)$ is because $\xi_{11}$ is non-negative. Note that the above equation contains $\xi_{11}, \xi_{22}, \xi_{21}$ which we would like to convert to the form $\left|\xi_{11}\right|^{2},\left|\xi_{22}\right|^{2},\left|\xi_{21}\right|^{2}$ which are available from 47, , 48, and (49). We handle $h\left(\left.\xi_{21}|| \xi_{11}\right|^{2}\right)$ with the following lemma.

Lemma 18. $h\left(\left.\xi_{21}|| \xi_{11}\right|^{2}\right) \leq h\left(\left.\left|\xi_{21}\right|^{2}|| \xi_{11}\right|^{2}\right)+\log (\pi)$.

Proof: We have

$$
\begin{aligned}
h\left(\left.\xi_{21}|| \xi_{11}\right|^{2}\right) & \stackrel{(i)}{=} h\left(\left.\xi_{21} e^{i \theta}|| \xi_{11}\right|^{2}, \theta\right) \\
& \stackrel{(i i)}{\leq} h\left(\left.\xi_{21} e^{i \theta}|| \xi_{11}\right|^{2}\right) \\
& \stackrel{(i i i)}{=} h\left(\left.\left|\xi_{21}\right|^{2}|| \xi_{11}\right|^{2}\right)+\log (\pi),
\end{aligned}
$$

where $(i)$ uses $\theta \sim \operatorname{Unif}[0,2 \pi]$ independent of the other random variables, $(i i)$ is because conditioning reduces entropy, (iii) is using Lemma 13 since given $\left|\xi_{11}\right|^{2}, \xi_{21} e^{i \theta}$ is isotropically distributed.

Using the above lemma, we get

$$
\begin{aligned}
h(Y) \leq & h\left(\xi_{11}{\overline{q_{1}}}^{(T)}\right)+h\left(\left.\xi_{22} \bar{q}_{2}^{(T-1)}|| \xi_{11}\right|^{2}\right)+h\left(\left.\left|\xi_{21}\right|^{2}|| \xi_{11}\right|^{2}\right)+\log (\pi) \\
\stackrel{(i)}{\leq} h & \left(\left|a g_{11}+b g_{12}+w_{11}\right|^{2}+\left|c g_{12}+w_{12}\right|^{2}+\sum_{i=3}^{T}\left|w_{1 i}\right|^{2}\right) \\
& +(T-1) \mathbb{E}\left[\log \left(|a|^{2} \rho_{11}^{2}+\left(|b|^{2}+|c|^{2}\right) \rho_{12}^{2}+1\right)\right]+\log \left(\frac{\pi^{T}}{\Gamma(T)}\right) \\
& +h\left(\left.\left|\xi_{21}\right|^{2}|| \xi_{11}\right|^{2}\right)+h\left(\left.\left|\xi_{22}\right|^{2}|| \xi_{11}\right|^{2}\right)+(T-2) \mathbb{E}\left[\log \left(\left|\xi_{22}\right|^{2}\right)\right]+\log (\pi),
\end{aligned}
$$

where $(i)$ is by applying Lemma 13 on $h\left(\xi_{11}{\overline{q_{1}}}^{(T)}\right)$ and Corollary 14 on $h\left(\left.\xi_{22} \bar{q}_{2}^{(T-1)}|| \xi_{11}\right|^{2}\right)$. Now we use the following Lemma to simplify $h\left(\left|a g_{11}+b g_{12}+w_{11}\right|^{2}+\left|c g_{12}+w_{12}\right|^{2}+\sum_{i=3}^{T}\left|w_{1 i}\right|^{2}\right)$ from the previous expression.

Lemma 19. For any given distribution on $(a, b, c)$, the terms

$$
h\left(\left|a g_{11}+b g_{12}+w_{11}\right|^{2}+\left|c g_{12}+w_{12}\right|^{2}+\sum_{i=3}^{T}\left|w_{1 i}\right|^{2}\right)
$$


and

$$
\mathbb{E}\left[\log \left(|a|^{2} \rho_{11}^{2}+\left(|b|^{2}+|c|^{2}\right) \rho_{12}^{2}+1\right)\right]
$$

have the same gDoF. Similarly for any given distribution on $(a, b, c)$, the terms

$$
h\left(\left|a g_{21}+b g_{22}+w_{21}\right|^{2}+\left|c g_{22}+w_{22}\right|^{2}+\sum_{i=3}^{T}\left|w_{2 i}\right|^{2}\right)
$$

and

$$
\mathbb{E}\left[\log \left(|a|^{2} \rho_{21}^{2}+\left(|b|^{2}+|c|^{2}\right) \rho_{22}^{2}+1\right)\right]
$$

have the same gDoF.

Proof: The proof proceeds by constructing a noncoherent channel

$$
\mathcal{C}_{1}: V=\left|a g_{11}+b g_{12}+w_{11}\right|^{2}+\left|c g_{12}+w_{12}\right|^{2}+\sum_{i=3}^{T}\left|w_{1 i}\right|^{2}
$$

with inputs $a, b, c$ and output $V$. Then we show that this channel has zero gDoF. The proof uses outer bounding techniques from [7]. See Appendix $\mathrm{G}$ for details.

Hence using the previous lemma, we get

$$
\begin{aligned}
h(Y) \leq & T \mathbb{E}\left[\log \left(|a|^{2} \rho_{11}^{2}+\left(|b|^{2}+|c|^{2}\right) \rho_{12}^{2}+1\right)\right] \\
& +h\left(\left.\left|\xi_{21}\right|^{2}\left|\xi_{11}\right|^{2}|| \xi_{11}\right|^{2}\right)+h\left(\left.\left|\xi_{22}\right|^{2}\left|\xi_{11}\right|^{2}|| \xi_{11}\right|^{2}\right) \\
& +(T-2) \mathbb{E}\left[\log \left(\left|\xi_{22}\right|^{2}\left|\xi_{11}\right|^{2}\right)\right]-T \mathbb{E}\left[\log \left(\left|\xi_{11}\right|^{2}\right)\right] .
\end{aligned}
$$

Now we simplify $\mathbb{E}\left[\log \left(\left|\xi_{11}\right|^{2}\right)\right]$ from the previous expression.

$$
\begin{aligned}
\mathbb{E}\left[\log \left(\left|\xi_{11}\right|^{2}\right)\right] & =\mathbb{E}\left[\log \left(\left|a g_{11}+b g_{12}+w_{11}\right|^{2}+\left|c g_{12}+w_{12}\right|^{2}+\sum_{i=3}^{T}\left|w_{1 i}\right|^{2}\right)\right] \\
& =\mathbb{E}\left[\mathbb{E}\left[\log \left(\left|a g_{11}+b g_{12}+w_{11}\right|^{2}+\left|c g_{12}+w_{12}\right|^{2}+\sum_{i=3}^{T}\left|w_{1 i}\right|^{2}\right) \mid a, b, c\right]\right] \\
& \stackrel{(i)}{=} \mathbb{E}\left[\log \left(|a|^{2} \rho_{11}^{2}+\left(|b|^{2}+|c|^{2}\right) \rho_{12}^{2}+1\right)\right],
\end{aligned}
$$

where $(i)$ was using Lemma 9 on page 16 and using the fact that $\left|a g_{11}+b g_{12}+w_{11}\right|^{2},\left|c g_{12}+w_{12}\right|^{2},\left|w_{1 i}\right|^{2}$ are exponentially distributed given $a, b, c$. Hence

$$
\begin{aligned}
h(Y) \leq & \left(\left.\left|\xi_{21}\right|^{2}\left|\xi_{11}\right|^{2}|| \xi_{11}\right|^{2}\right)+h\left(\left.\left|\xi_{22}\right|^{2}\left|\xi_{11}\right|^{2}|| \xi_{11}\right|^{2}\right) \\
& +(T-2) \mathbb{E}\left[\log \left(\left|\xi_{22}\right|^{2}\left|\xi_{11}\right|^{2}\right)\right] .
\end{aligned}
$$


Now we use the following lemmas to further simplify the terms in the above expression for $h(Y)$.

Lemma 20. For any given distribution on $(a, b, c)$,

$$
h\left(\left.\left|\xi_{22}\right|^{2}\left|\xi_{11}\right|^{2}|| \xi_{11}\right|^{2}\right) \doteq h\left(\left.\left|\xi_{22}\right|^{2}\left|\xi_{11}\right|^{2}|| \xi_{11}\right|^{2}, a, b, c\right) \leq \mathbb{E}\left[\log \left(e \mathbb{E}\left[\left|\xi_{22}\right|^{2}\left|\xi_{11}\right|^{2} \mid a, b, c\right]\right)\right] .
$$

Proof: The proof uses similar techniques like that for Lemma 19. See Appendix $\mathrm{H}$ for details.

Lemma 21. For any given distribution on $(a, b, c)$,

$$
h\left(\left.\left|\xi_{21}\right|^{2}\left|\xi_{11}\right|^{2}|| \xi_{11}\right|^{2}\right) \doteq h\left(\left.\left|\xi_{21}\right|^{2}\left|\xi_{11}\right|^{2}|| \xi_{11}\right|^{2}, a, b, c\right) \leq \mathbb{E}\left[\log \left(e \mathbb{E}\left[\left|\xi_{21}\right|^{2}\left|\xi_{11}\right|^{2} \mid a, b, c\right]\right)\right] .
$$

Proof: This can be proved similar to the previous lemma. We omit the proof.

We have

$\left|\xi_{21}\right|^{2}\left|\xi_{11}\right|^{2}=\left|\left(a g_{21}+b g_{22}+w_{21}\right)\left(a g_{11}+b g_{12}+w_{11}\right)^{*}+\left(c g_{22}+w_{22}\right)\left(c g_{12}+w_{12}\right)^{*}+\sum_{i=3}^{T} w_{2 i} w_{1 i}^{*}\right|^{2}$.

Hence using 47, , 48, and Lemma 21 to bound $h\left(\left.\left|\xi_{21}\right|^{2}\left|\xi_{11}\right|^{2}|| \xi_{11}\right|^{2}\right)$, we get

$$
\begin{aligned}
& h\left(\left.\left|\xi_{21}\right|^{2}\left|\xi_{11}\right|^{2}|| \xi_{11}\right|^{2}\right) \\
& \dot{\leq} \mathbb{E}\left[\operatorname { l o g } \left(\left(|a|^{2} \rho_{11}^{2}+|b|^{2} \rho_{12}^{2}+1\right)\left(|a|^{2} \rho_{21}^{2}+|b|^{2} \rho_{22}^{2}+1\right)\right.\right. \\
& \left.\left.\quad+2|c|^{2}|b|^{2} \rho_{22}^{2} \rho_{12}^{2}+\left(|c|^{2} \rho_{12}^{2}+1\right)\left(|c|^{2} \rho_{22}^{2}+1\right)+T-2\right)\right] \\
& \dot{\leq} \mathbb{E}\left[\operatorname { l o g } \left(\left(|a|^{2} \rho_{11}^{2}+|b|^{2} \rho_{12}^{2}+1\right)\left(|a|^{2} \rho_{21}^{2}+|b|^{2} \rho_{22}^{2}+1\right)\right.\right. \\
& \left.\left.+\left(|c|^{2} \rho_{12}^{2}+1\right)\left(|c|^{2} \rho_{22}^{2}+1\right)\right)\right]
\end{aligned}
$$

where the last step followed due to AM-GM inequality (arithmetic mean $\leq$ geometric mean). The AM-GM inequality yields $2|c|^{2}|b|^{2} \rho_{22}^{2} \rho_{12}^{2} \leq|b|^{4} \rho_{22}^{2} \rho_{12}^{2}+|c|^{4} \rho_{22}^{2} \rho_{12}^{2}$. Similarly, using (47) and (49), we have

$$
\begin{aligned}
& \left|\xi_{22}\right|^{2}\left|\xi_{11}\right|^{2} \\
& =\left(\left|a g_{21}+b g_{22}+w_{21}\right|^{2}+\left|c g_{22}+w_{22}\right|^{2}+\sum_{i=3}^{T}\left|w_{2 i}\right|^{2}\right)\left(\left|a g_{11}+b g_{12}+w_{11}\right|^{2}+\left|c g_{12}+w_{12}\right|^{2}+\sum_{i=3}^{T}\left|w_{1 i}\right|^{2}\right) \\
& \quad-\left|\left(a g_{21}+b g_{22}+w_{21}\right)\left(a g_{11}+b g_{12}+w_{11}\right)^{*}+\left(c g_{22}+w_{22}\right)\left(c g_{12}+w_{12}\right)^{*}+\sum_{i=3}^{T} w_{2 i} w_{1 i}^{*}\right|^{2} .
\end{aligned}
$$


After some algebraic manipulations, it can be seen that

$$
\begin{aligned}
& \mathbb{E}\left[\left|\xi_{22}\right|^{2}\left|\xi_{11}\right|^{2} \mid a, b, c\right] \\
& =(T-2)^{2}-(T-2)+(T-2)\left(|a|^{2} \rho_{21}^{2}+|b|^{2} \rho_{22}^{2}+|c|^{2} \rho_{22}^{2}+|a|^{2} \rho_{11}^{2}+|b|^{2} \rho_{12}^{2}+|c|^{2} \rho_{12}^{2}+2\right) \\
& \quad+\left(|a|^{2} \rho_{11}^{2}+1\right)\left(|c|^{2} \rho_{22}^{2}+1\right)+|b|^{2} \rho_{12}^{2}+\left(|a|^{2} \rho_{21}^{2}+1\right)\left(|c|^{2} \rho_{12}^{2}+1\right)+|b|^{2} \rho_{22}^{2} .
\end{aligned}
$$

After retaining only the terms that contribute to $\mathrm{gDoF}$ from the above equation, we bound $h\left(\left.\left|\xi_{22}\right|^{2}\left|\xi_{11}\right|^{2}|| \xi_{11}\right|^{2}\right)$ using Lemma 21 to get

$$
\begin{aligned}
& h\left(\left.\left|\xi_{22}\right|^{2}\left|\xi_{11}\right|^{2}|| \xi_{11}\right|^{2}\right) \leq \mathbb{E}\left[\log \left(e \mathbb{E}\left[\left|\xi_{22}\right|^{2}\left|\xi_{11}\right|^{2} \mid a, b, c\right]\right)\right] \\
& \leq \mathbb{E}\left[\operatorname { l o g } \left(\left(|a|^{2} \rho_{11}^{2}+1\right)\left(|c|^{2} \rho_{22}^{2}+1\right)+|b|^{2}\left(\rho_{12}^{2}+\rho_{22}^{2}\right)\right.\right. \\
&\left.\left.+\left(|a|^{2} \rho_{21}^{2}+1\right)\left(|c|^{2} \rho_{12}^{2}+1\right)\right)\right] .
\end{aligned}
$$

Also

$$
\begin{aligned}
\mathbb{E}\left[\log \left(\left|\xi_{22}\right|^{2}\left|\xi_{11}\right|^{2}\right)\right] \leq \mathbb{E}\left[\log \left(\mathbb{E}\left[\left|\xi_{22}\right|^{2}\left|\xi_{11}\right|^{2} \mid a, b, c\right]\right)\right] \\
\leq \mathbb{E}\left[\operatorname { l o g } \left(\left(|a|^{2} \rho_{11}^{2}+1\right)\left(|c|^{2} \rho_{22}^{2}+1\right)+|b|^{2}\left(\rho_{12}^{2}+\rho_{22}^{2}\right)\right.\right. \\
\left.\left.+\left(|a|^{2} \rho_{21}^{2}+1\right)\left(|c|^{2} \rho_{12}^{2}+1\right)\right)\right] .
\end{aligned}
$$

Hence using (72), (71), 69) in (66), we get

$$
\begin{gathered}
h(Y) \\
\dot{\leq} \mathbb{E}\left[\log \left(\left(|a|^{2} \rho_{11}^{2}+|b|^{2} \rho_{12}^{2}+1\right)\left(|a|^{2} \rho_{21}^{2}+|b|^{2} \rho_{22}^{2}+1\right)+\left(|c|^{2} \rho_{12}^{2}+1\right)\left(|c|^{2} \rho_{22}^{2}+1\right)\right)\right] \\
+(T-1) \mathbb{E}\left[\operatorname { l o g } \left(\left(|a|^{2} \rho_{11}^{2}+1\right)\left(|c|^{2} \rho_{22}^{2}+1\right)+|b|^{2}\left(\rho_{12}^{2}+\rho_{22}^{2}\right)\right.\right. \\
\left.\left.+\left(|a|^{2} \rho_{21}^{2}+1\right)\left(|c|^{2} \rho_{12}^{2}+1\right)\right)\right] .
\end{gathered}
$$

Using the above equation and (50), we get

$$
\begin{aligned}
& I(X ; Y) \\
& \dot{\leq} \mathbb{E}\left[\log \left(\left(|a|^{2} \rho_{11}^{2}+|b|^{2} \rho_{12}^{2}+1\right)\left(|a|^{2} \rho_{21}^{2}+|b|^{2} \rho_{22}^{2}+1\right)+\left(|c|^{2} \rho_{12}^{2}+1\right)\left(|c|^{2} \rho_{22}^{2}+1\right)\right)\right] \\
& \quad+(T-1) \mathbb{E}\left[\log \left(\left(|a|^{2} \rho_{11}^{2}+1\right)\left(|c|^{2} \rho_{22}^{2}+1\right)+|b|^{2}\left(\rho_{12}^{2}+\rho_{22}^{2}\right)+\left(|a|^{2} \rho_{21}^{2}+1\right)\left(|c|^{2} \rho_{12}^{2}+1\right)\right)\right] \\
& \quad-\mathbb{E}\left[\log \left(|a|^{2} \rho_{11}^{2}+|b|^{2} \rho_{12}^{2}+|c|^{2} \rho_{12}^{2}+|a|^{2}|c|^{2} \rho_{11}^{2} \rho_{12}^{2}+1\right)\right] \\
& \quad-\mathbb{E}\left[\log \left(|a|^{2} \rho_{21}^{2}+|b|^{2} \rho_{22}^{2}+|c|^{2} \rho_{22}^{2}+|a|^{2}|c|^{2} \rho_{21}^{2} \rho_{22}^{2}+1\right)\right]
\end{aligned}
$$




$$
=\mathbb{E}\left[f\left(|a|^{2},|b|^{2},|c|^{2}\right)\right]
$$

where the last step included a trivial definition for $f\left(|a|^{2},|b|^{2},|c|^{2}\right)$, by collecting all the terms from the previous equation. Hence an outer bound for the gDoF of the channel can be obtained by solving the following optimization problem:

$$
\mathcal{P}_{1}:\left\{\underset{\mathbb{E}\left[|a|^{2}+|b|^{2}+|c|^{2}\right] \leq T}{\operatorname{maximize}} \mathbb{E}\left[f\left(|a|^{2},|b|^{2},|c|^{2}\right)\right] .\right.
$$

Now we use the following lemma to simplify $\mathcal{P}_{1}$ without losing gDoF.

Lemma 22. The gDoF achieved in $\mathcal{P}_{1}$ can be achieved by a point mass distribution, i.e.,

$$
g \operatorname{DoF}\left(\mathcal{P}_{1}\right)=g \operatorname{DoF}\left(\mathcal{P}_{7}\right)
$$

where $\mathcal{P}_{7}$ is the following:

$$
\mathcal{P}_{7}:\left\{\begin{array}{l}
\text { maximize } f\left(|a|^{2},|b|^{2},|c|^{2}\right) \quad \text { with } \\
|a|^{2} \leq T,|b|^{2} \leq T,|c|^{2} \leq T
\end{array}\right.
$$

Proof idea: The proof proceeds in several steps:

Step 1: Show that there exists a discretization (over an infinite set) for any distribution of $\left(|a|^{2},|b|^{2},|c|^{2}\right)$ that does not incur a loss in gDoF.

Step 2: Show that the discretization can be limited to a finite set without incurring a loss in gDoF.

Step 3: View the problem as a linear program with two constraints, and show that there is an optimal distribution with just two mass points.

Step 4: Show that the two mass points can be collapsed to a single point using arguments of symmetry.

The details of the proof are given in Appendix $B$.

Changing the variables from $\left(|a|^{2},|b|^{2},|c|^{2}\right)$ to $\left(\gamma_{a}, \gamma_{b}, \gamma_{c}\right)$ with the substitution $|a|^{2}=\mathrm{SNR}^{-\gamma_{a}}$, $|b|^{2}=\mathrm{SNR}^{-\gamma_{b}},|c|^{2}=\mathrm{SNR}^{-\gamma_{c}}$, it is clear that

$$
\operatorname{gDoF}\left(\mathcal{P}_{1}\right)=\operatorname{gDoF}\left(\mathcal{P}_{7}\right)=\left(\mathcal{P}_{8}\right),
$$

where $\mathcal{P}_{8}$ is the following:

$$
\mathcal{P}_{8}:\left\{\begin{array}{l}
\operatorname{maximize} f_{\gamma}\left(\gamma_{a}, \gamma_{b}, \gamma_{c}\right) \\
\gamma_{a} \geq 0, \gamma_{b} \geq 0, \gamma_{c} \geq 0
\end{array}\right.
$$


with

$$
\begin{aligned}
& f_{\gamma}\left(\gamma_{a}, \gamma_{b}, \gamma_{c}\right) \\
& =\max \left(\max \left(-\gamma_{a}+\gamma_{11},-\gamma_{b}+\gamma_{12}, 0\right)+\max \left(-\gamma_{a}+\gamma_{21},-\gamma_{b}+\gamma_{22}, 0\right),\right. \\
& \left.\max \left(-\gamma_{c}+\gamma_{12}, 0\right)+\max \left(-\gamma_{c}+\gamma_{22}, 0\right)\right) \\
& +(T-1) \max \left(\max \left(-\gamma_{a}+\gamma_{11}, 0\right)+\max \left(-\gamma_{c}+\gamma_{22}, 0\right),\right. \\
& \left.\quad \gamma_{b}+\max \left(\gamma_{12}, \gamma_{22}\right), \max \left(-\gamma_{a}+\gamma_{21}, 0\right)+\max \left(-\gamma_{c}+\gamma_{12}, 0\right)\right) \\
& -\max \left(-\gamma_{a}+\gamma_{11},-\gamma_{b}+\gamma_{12},-\gamma_{c}+\gamma_{12},-\gamma_{a}-\gamma_{c}+\gamma_{11}+\gamma_{12}, 0\right) \\
& \max \left(-\gamma_{a}+\gamma_{21},-\gamma_{b}+\gamma_{22},-\gamma_{c}+\gamma_{22},-\gamma_{a}-\gamma_{c}+\gamma_{21}+\gamma_{22}, 0\right) .
\end{aligned}
$$

For a $2 \times 2$ MIMO channel with two different SNR exponents, one in the direct links and another in the crosslinks, we have $\gamma_{11}=\gamma_{22}=\gamma_{D}, \gamma_{C L}=\gamma_{12}=\gamma_{21}$. Also, without loss of generality, it can be assumed that $\gamma_{D}>\gamma_{C L}$. By inspection of the optimization problem, it is clear that we can also restrict $\gamma_{a} \leq \gamma_{D}, \gamma_{b} \leq \gamma_{D}, \gamma_{c} \leq \gamma_{D}$ without affecting the solution. With these additional constraints, we can simplify $\mathcal{P}_{8}$ to $\mathcal{P}_{9}$ with $\mathcal{P}_{9}$ defined as the following:

$$
\mathcal{P}_{9}:\left\{\begin{array}{c}
\operatorname{maximize} \max \left(-2 \gamma_{a}+\gamma_{C L},-\gamma_{a}+\gamma_{D}-\gamma_{b},-2 \gamma_{b}+\gamma_{C L},-2 \gamma_{c}+\gamma_{C L},-\gamma_{c}\right) \\
\quad+(T-1) \max \left(-\gamma_{a}+\gamma_{D}-\gamma_{c},-\gamma_{b}\right)+T \gamma_{D}-t_{1}-t_{2} \\
t_{1}=\max \left(-\gamma_{a}+\gamma_{D},-\gamma_{b}+\gamma_{C L},-\gamma_{a}-\gamma_{c}+\gamma_{D}+\gamma_{C L}\right) \\
t_{2}=\max \left(-\gamma_{b}+\gamma_{D},-\gamma_{c}+\gamma_{D},-\gamma_{a}-\gamma_{c}+\gamma_{D}+\gamma_{C L}\right) \\
0 \leq \gamma_{a} \leq \gamma_{D}, 0 \leq \gamma_{b} \leq \gamma_{D}, 0 \leq \gamma_{c} \leq \gamma_{D} .
\end{array}\right.
$$

Using standard linear programming arguments, $\mathcal{P}_{9}$ has a solution for $\left(\gamma_{a}, \gamma_{b}, \gamma_{c}, t_{1}, t_{2}\right)$ in one of the corner points of the following region:

$$
\mathcal{R}:\left\{\begin{array}{c}
0 \leq \gamma_{a} \leq \gamma_{D} ; 0 \leq \gamma_{b} \leq \gamma_{D} \\
0 \leq \gamma_{c} \leq \gamma_{D} \\
t_{1} \geq-\gamma_{a}+\gamma_{D} ; t_{1} \geq-\gamma_{b}+\gamma_{C L} \\
t_{1} \geq-\gamma_{a}-\gamma_{c}+\gamma_{D}+\gamma_{C L} ; t_{2} \geq-\gamma_{b}+\gamma_{D} \\
t_{2} \geq-\gamma_{a}-\gamma_{c}+\gamma_{D}+\gamma_{C L} ; t_{2} \geq-\gamma_{c}+\gamma_{D}
\end{array}\right\}
$$


This can be seen by considering case by case for $\mathcal{P}_{9}$, depending on which term inside the $\max (\cdot)$ 's could come out in the objective function, and noting that $\operatorname{maximize}_{\gamma_{a}, \gamma_{b}, \gamma_{c}, t_{1}, t_{2}} \max \left(f_{1}, f_{2}\right)$ is same as $\max \left(\operatorname{maximize}_{\gamma_{a}, \gamma_{b}, \gamma_{c}, t_{1}, t_{2}}\left(f_{1}\right), \underset{\gamma_{a}, \gamma_{b}, \gamma_{c}, t_{1}, t_{2}}{\operatorname{maximize}}\left(f_{2}\right)\right)$ for linear $f_{1}, f_{2}$.

$$
\text { Suppose }-2 \gamma_{a}+\gamma_{C L}=\max \left(-2 \gamma_{a}+\gamma_{C L},-\gamma_{a}+\gamma_{D}-\gamma_{b},-2 \gamma_{b}+\gamma_{C L},-2 \gamma_{c}+\gamma_{C L},-\gamma_{c}\right)
$$
and $-\gamma_{a}+\gamma_{D}-\gamma_{c}=\max \left(-\gamma_{a}+\gamma_{D}-\gamma_{c},-\gamma_{b}\right)$, then $\mathcal{P}_{9}$ has a solution in one of the corner points of $\mathcal{R}$. This is true for all possible cases of the values of the two $\max \left(\right.$ )'s. Hence $\mathcal{P}_{9}$ itself has a solution in one of the corner points of $\mathcal{R}$.

We code in Mathematica to find all the corner points of $\mathcal{R}$ and find the maximum across the corner points. Finding all the corner points and the subsequent calculations are mechanical, and we believe, does not add value to the paper and would only further lengthen the paper. So we have deferred it to the software. The result is still mathematically rigorous, and we suggest the use of Mathematica to verify some formulas rather than perform long calculations. We obtain the solution in Table I. Our Mathematica code is available online at https://arxiv.org/src/1705. 07355v5/anc/Sym_mimo_outerbound.nb. This code uses $\gamma_{D}=1, \gamma_{C L}=1-\epsilon$ and we can obtain the general solution with a simple scaling.

\section{Conclusions}

We considered the noncoherent MIMO channel with link strengths scaled with different exponents of SNR. Under this model, we derived a structure for the capacity achieving input distribution. We showed that for $T=1$, the $\mathrm{gDoF}$ is zero for a MIMO channel of any size. Also for SIMO channels and MISO channels, we proved that selecting the best antenna can achieve the gDoF. We derived the $\mathrm{gDoF}$ for the $2 \times 2 \mathrm{MIMO}$ channel with two different exponents in the direct and cross links and showed that both the antennas are always needed to achieve the gDoF. Also, training-based schemes were shown to be suboptimal for this $2 \times 2 \mathrm{MIMO}$ channel with two different exponents. We extended this observation to an $M \times M$ MIMO channel with two different exponents in the direct and cross links; we demonstrated a strategy that could achieve larger $\mathrm{gDoF}$ than training-based schemes. A possible direction for future work would be to try to derive the $\mathrm{gDoF}$ of $M \times M$ MIMO channels with two different exponents in the direct and cross links. A subsequent step would be to look into the case of MIMO channels with arbitrary size and arbitrary SNR exponents. The outer bounds for larger MIMO channels seem to be a challenge at the moment, our outer bounds for the $2 \times 2$ MIMO channel illustrate some of the difficulties: we used a Gram-Schmidt process for the LQ decomposition of matrices 
and developed new lemmas to bound the terms in the mutual information expression. The same methods do not seem to be directly applicable to larger MIMO systems. Another line of work is to study the gDoF for noncoherent relay channels.

\section{APPENDIX A \\ PROOF OF LEMMA 13}

Here we derive the formula for calculating $h\left(\left[\xi_{1}, \xi_{2}, \ldots, \xi_{n}\right] Q\right)$ with $\left[\xi_{1}, \xi_{2}, \ldots, \xi_{n}\right]$ being an arbitrary complex random vector and $Q$ being an $n \times n$ isotropically distributed random unitary matrix independent of $\xi_{i}$. We do this by noting that in radial coordinates, the distribution of $\left[\xi_{1}, \xi_{2}, \ldots, \xi_{n}\right] Q$ is dependent only on the radius. Let

$$
V=\left[\xi_{1}, \xi_{2}, \ldots, \xi_{n}\right] Q
$$

Now for any fixed $n \times n$ unitary matrix $Q^{\prime}$, the vectors $V$ and $V Q^{\prime}$ have the same distribution due to the property of isotropic distribution. Hence for any $v_{1}, v_{2} \in \mathbb{C}^{n}$, if $\left\|v_{1}\right\|=\left\|v_{2}\right\|$, then

$$
p_{v}\left(v_{1}\right)=p_{v}\left(v_{2}\right)
$$

since there exists a unitary matrix $Q^{\prime \prime}$ such that $v_{1} Q^{\prime \prime}=v_{2}$. One such $Q^{\prime \prime}$ can be obtained using Householder transformation. Now the probability distribution can be viewed in $\mathbb{R}^{2 n}$, and we use the $2 n$ dimensional vector $U$. Let

$$
\Upsilon=\sum\left|\xi_{i}\right|^{2}
$$

Let $(r, \bar{\theta})$ be the radial coordinates, $(t, \bar{\theta})$ be similar coordinates but with $t=r^{2}$. Let $p_{u, t}(t, \bar{\theta})=$ $p_{u}(u(t, \bar{\theta}))$ be obtained from $p_{u}(u)$ by expressing $u$ in $(t, \bar{\theta})$ coordinates. Similarly $p_{u, r}(r, \bar{\theta})=$ $p_{u}(u(r, \bar{\theta}))$.

The $2 n-1$ dimensional surface area (embedded in a $2 n$ dimensional Euclidean) is $\left(\frac{2 \pi^{n}}{\Gamma(n)}\right) r^{2 n-1}$. Hence

$$
\left(\frac{2 \pi^{n}}{\Gamma(n)}\right) p_{u, r}(r, \bar{\theta}) r^{2 n-1} d r
$$

is the probability that $|U| \in[r, r+d r]$. Hence $\left(\frac{\pi^{n}}{\Gamma(n)}\right) p_{u, t}(t, \bar{\theta}) t^{n-1} d t$ is the probability that $\Upsilon=\|U\|^{2} \in[t, t+d t]$. Hence

$$
\begin{aligned}
\left(\frac{\pi^{n}}{\Gamma(n)}\right) p_{u, t}(t, \bar{\theta}) t^{n-1} & =p_{\Upsilon}(t) \\
p_{u, t}(t, \bar{\theta}) & =p_{\Upsilon}(t) \frac{1}{t^{n-1}\left(\frac{\pi^{n}}{\Gamma(n)}\right)} .
\end{aligned}
$$


Now

$$
\begin{aligned}
h(U) & =-\int p_{u}(u) \log \left(p_{u}(u)\right) d u \\
& \stackrel{(i)}{=}-\int p_{u}(u(r, \bar{\theta})) \log \left(p_{u}(u(r, \bar{\theta}))\right)\left(\frac{2 \pi^{n}}{\Gamma(n)}\right) r^{2 n-1} d r \\
& \stackrel{(i i)}{=}-\int p_{u}(u(t, \bar{\theta})) \log \left(p_{u}(u(t, \bar{\theta}))\right)\left(\frac{\pi^{n}}{\Gamma(n)}\right) t^{n-1} d t \\
& \stackrel{(i i i)}{=}-\int p_{\Upsilon}(t) \log \left(p_{\Upsilon}(t) \frac{1}{t^{n-1}\left(\frac{\pi^{n}}{\Gamma(n)}\right)}\right) d t \\
& =-\int p_{\Upsilon}(t) \log \left(p_{\Upsilon}(t)\right) d t+\log \left(\frac{\pi^{n}}{\Gamma(n)}\right)+(n-1) \int p_{\Upsilon}(t) \log (t) d t \\
& =h(\Upsilon)+(n-1) \mathbb{E}[\log (\Upsilon)]+\log \left(\frac{\pi^{n}}{\Gamma(n)}\right) \\
& =h\left(\sum\left|\xi_{i}\right|^{2}\right)+(n-1) \mathbb{E}\left[\log \left(\sum\left|\xi_{i}\right|^{2}\right)\right]+\log \left(\frac{\pi^{n}}{\Gamma(n)}\right),
\end{aligned}
$$

where $(i)$ is by change of variables to $(r, \bar{\theta})$, then integrating over $\bar{\theta}$ and noting that $p_{u}(u(r, \bar{\theta}))$ is independent of $\bar{\theta}$. The step $(i i)$ is by change of variables to $(t, \bar{\theta}),(i i i)$ is using 84 .

\section{APPENDIX B}

\section{PROOF OF LEMMA 22}

Here we consider the optimization problem $\mathcal{P}_{1}$ from $(76)$ on page 29 and show that its objective function $\mathbb{E}\left[f\left(|a|^{2},|b|^{2},|c|^{2}\right)\right]$ can be optimized for $\mathrm{gDoF}$ by a point mass distribution. We have the form for $f\left(|a|^{2},|b|^{2},|c|^{2}\right)$ as

$$
\begin{aligned}
& f\left(|a|^{2},|b|^{2},|c|^{2}\right) \\
& =\log \left(\left(|a|^{2} \rho_{11}^{2}+|b|^{2} \rho_{12}^{2}+1\right)\left(|a|^{2} \rho_{21}^{2}+|b|^{2} \rho_{22}^{2}+1\right)+\left(|c|^{2} \rho_{12}^{2}+1\right)\left(|c|^{2} \rho_{22}^{2}+1\right)\right) \\
& \quad+(T-1) \log \left(\left(|a|^{2} \rho_{11}^{2}+1\right)\left(|c|^{2} \rho_{22}^{2}+1\right)+|b|^{2}\left(\rho_{12}^{2}+\rho_{22}^{2}\right)+\left(|a|^{2} \rho_{21}^{2}+1\right)\left(|c|^{2} \rho_{12}^{2}+1\right)\right) \\
& \quad-\log \left(\left(1+|a|^{2} \rho_{11}^{2}\right)\left(1+|c|^{2} \rho_{12}^{2}\right)+|b|^{2} \rho_{12}^{2}\right) \\
& \quad-\log \left(\left(1+|a|^{2} \rho_{21}^{2}\right)\left(1+|c|^{2} \rho_{22}^{2}\right)+|b|^{2} \rho_{22}^{2}\right) .
\end{aligned}
$$

Now

$$
\begin{aligned}
& \frac{\partial}{\partial|a|^{2}} f\left(|a|^{2},|b|^{2},|c|^{2}\right) \\
& =\frac{\rho_{11}^{2}\left(|a|^{2} \rho_{21}^{2}+|b|^{2} \rho_{22}^{2}+1\right)+\left(|a|^{2} \rho_{11}^{2}+|b|^{2} \rho_{12}^{2}+1\right) \rho_{21}^{2}}{\left(|a|^{2} \rho_{11}^{2}+|b|^{2} \rho_{12}^{2}+1\right)\left(|a|^{2} \rho_{21}^{2}+|b|^{2} \rho_{22}^{2}+1\right)+\left(|c|^{2} \rho_{12}^{2}+1\right)\left(|c|^{2} \rho_{22}^{2}+1\right)}
\end{aligned}
$$




$$
\begin{aligned}
& +(T-1) \frac{\rho_{11}^{2}\left(|c|^{2} \rho_{22}^{2}+1\right)+\left(|c|^{2} \rho_{12}^{2}+1\right) \rho_{21}^{2}}{\left(|a|^{2} \rho_{11}^{2}+1\right)\left(|c|^{2} \rho_{22}^{2}+1\right)+|b|^{2}\left(\rho_{12}^{2}+\rho_{22}^{2}\right)+\left(|a|^{2} \rho_{21}^{2}+1\right)\left(|c|^{2} \rho_{12}^{2}+1\right)} \\
& -\frac{\left(\rho_{11}^{2}\right)\left(1+|c|^{2} \rho_{12}^{2}\right)}{\left(1+|a|^{2} \rho_{11}^{2}\right)\left(1+|c|^{2} \rho_{12}^{2}\right)+|b|^{2} \rho_{12}^{2}} \\
& -\frac{\left(\rho_{21}^{2}\right)\left(1+|c|^{2} \rho_{22}^{2}\right)}{\left(1+|a|^{2} \rho_{21}^{2}\right)\left(1+|c|^{2} \rho_{22}^{2}\right)+|b|^{2} \rho_{22}^{2}}
\end{aligned}
$$

Hence

$$
\begin{aligned}
\left|\frac{\partial}{\partial|a|^{2}} f\left(|a|^{2},|b|^{2},|c|^{2}\right)\right| & \leq \rho_{11}^{2}+\rho_{21}^{2}+(T-1)\left(\rho_{11}^{2}+\rho_{21}^{2}\right)+\left(\rho_{11}^{2}\right)+\left(\rho_{21}^{2}\right) \\
& \leq 2(T+1) \max _{i, j} \rho_{i j}^{2} .
\end{aligned}
$$

Similarly

$$
\begin{aligned}
\left|\frac{\partial}{\partial|b|^{2}} f\left(|a|^{2},|b|^{2},|c|^{2}\right)\right| & \leq 2(T+1) \max _{i, j} \rho_{i j}^{2}, \\
\left|\frac{\partial}{\partial|c|^{2}} f\left(|a|^{2},|b|^{2},|c|^{2}\right)\right| & \leq 2(T+1) \max _{i, j} \rho_{i j}^{2}
\end{aligned}
$$

holds. Let $\rho_{*}^{2}=\max _{i, j} \rho_{i j}^{2}$. Now with $\Delta=1 /\left(2(T+1) \rho_{*}^{2}\right)$, if $\left\|\left(|a|^{2},|b|^{2},|c|^{2}\right)-\left(\left|a^{\prime}\right|^{2},\left|b^{\prime}\right|^{2},\left|c^{\prime}\right|^{2}\right)\right\| \leq$ $\sqrt{3} \Delta$, then

$$
\begin{aligned}
& \left|f\left(|a|^{2},|b|^{2},|c|^{2}\right)-f\left(\left|a^{\prime}\right|^{2},\left|b^{\prime}\right|^{2},\left|c^{\prime}\right|^{2}\right)\right| \\
& \leq\left\|\left[2(T+1) \rho_{*}^{2}, 2(T+1) \rho_{*}^{2}, 2(T+1) \rho_{*}^{2}\right]\right\| \sqrt{3} \Delta \\
& \leq 3 .
\end{aligned}
$$

Hence by considering a discrete version of the problem as

$$
\mathcal{P}_{2}:\left\{\begin{array}{l}
\underset{\mathbb{E}\left[|a|^{2}+|b|^{2}+|c|^{2}\right] \leq T}{\operatorname{maximize}} \mathbb{E}\left[f\left(|a|^{2},|b|^{2},|c|^{2}\right)\right] \\
\operatorname{Support}\left(|a|^{2},|b|^{2},|c|^{2}\right)=\{0, \Delta, 2 \Delta, \ldots, \infty\}^{3}
\end{array}\right.
$$

the optimum value achieved is within 3 of the optimum value of $\mathcal{P}_{1}$. Hence for an outer bound on gDoF, it is sufficient to solve $\mathcal{P}_{2}$.

$$
\operatorname{gDoF}\left(\mathcal{P}_{1}\right)=\operatorname{gDoF}\left(\mathcal{P}_{2}\right) .
$$

We will now show that it is sufficient to restrict Support $\left(|a|^{2},|b|^{2},|c|^{2}\right)=\left\{0, \Delta, 2 \Delta, \ldots,\left\lfloor\rho_{*}^{4}\right\rfloor \Delta\right\}^{3}$ for an outer bound on gDoF. 
Let the optimum value of $\mathcal{P}_{2}$ be achieved by a probability distribution $\left\{p_{i}^{*}\right\}$ at the points $\left\{\left(l_{1 i}^{*} \Delta, l_{2 i}^{*} \Delta, l_{3 i}^{*} \Delta\right)\right\}$ with $l_{j i}^{*} \in \mathbb{Z}$. Let

$$
\begin{gathered}
S_{1}=\left\{i: \max \left(l_{1 i}^{*}, l_{2 i}^{*}, l_{3 i}^{*}\right) \leq \rho_{*}^{4}\right\}, \\
S_{2}=\left\{i: \max \left(l_{1 i}^{*}, l_{2 i}^{*}, l_{3 i}^{*}\right)>\rho_{*}^{4}\right\}
\end{gathered}
$$

and let $\max \left(l_{1 i}^{*}, l_{2 i}^{*}, l_{3 i}^{*}\right)=l_{M i}^{*}$ for labeling. The optimum value $\left(\mathcal{P}_{2}\right)$ is given by

$$
\left(\mathcal{P}_{2}\right)=\sum_{i \in S_{1}} p_{i}^{*} f\left(l_{1 i}^{*} \Delta, l_{2 i}^{*} \Delta, l_{3 i}^{*} \Delta\right)+\sum_{i \in S_{2}} p_{i}^{*} f\left(l_{1 i}^{*} \Delta, l_{2 i}^{*} \Delta, l_{3 i}^{*} \Delta\right) .
$$

We will now show that $\sum_{i \in S_{2}} p_{i}^{*} f\left(l_{1 i}^{*} \Delta, l_{2 i}^{*} \Delta, l_{3 i}^{*} \Delta\right)$ does not contribute to the gDoF. The points in $S_{2}$ have large power and hence they have low probability due to power constraints; this ends up limiting the contribution to $\mathrm{gDoF}$. We prove this precisely in the following steps. Using the structure of $f\left(|a|^{2},|b|^{2},|c|^{2}\right)$ and $\Delta=1 /\left(2(T+1) \rho_{*}^{2}\right)$, we can bound

$$
\begin{aligned}
\left|f\left(l_{1 i}^{*} \Delta, l_{2 i}^{*} \Delta, l_{3 i}^{*} \Delta\right)\right| \leq & \log \left(\left(2 l_{M i}^{*}+1\right)\left(2 l_{M i}^{*}+1\right)+\left(l_{M i}^{*}+1\right)\left(l_{M i}^{*}+1\right)\right) \\
& +(T-1) \log \left(\left(l_{M i}^{*}+1\right)\left(l_{M i}^{*}+1\right)+2 l_{M i}^{*}+\left(l_{M i}^{*}+1\right)\left(l_{M i}^{*}+1\right)\right) \\
& \quad+2 \log \left(\left(1+l_{M i}^{*}\right)\left(1+l_{M i}^{*}\right)+l_{M i}^{*}\right) \\
\leq & (T+2) \log \left(\left(2 l_{M i}^{*}+1\right)\left(2 l_{M i}^{*}+1\right) 3\right) \\
= & 2(T+2) \log \left(2 l_{M i}^{*}+1\right)+(T+2) \log (3)
\end{aligned}
$$

Hence

$$
\begin{aligned}
& \left|\sum_{i \in S_{2}} p_{i}^{*} f\left(l_{1 i}^{*} \Delta, l_{2 i}^{*} \Delta, l_{3 i}^{*} \Delta\right)\right| \\
& \leq \sum_{i \in S_{2}} p_{i}^{*} 2(T+2) \log \left(2 l_{M i}^{*}+1\right)+(T+2) \log (3) \\
& \stackrel{(i)}{\leq} 2(T+2)\left(\sum_{i \in S_{2}} p_{i}^{*}\right) \log \left(2 \frac{\sum_{i \in S_{2}} p_{i}^{*} l_{M i}^{*}}{\sum_{j \in S_{2}} p_{j}^{*}}+1\right)+(T+2) \log (3) \\
& \stackrel{(i i)}{\leq} 2(T+2)\left(\sum_{i \in S_{2}} p_{i}^{*}\right) \log \left(2 \frac{T}{\Delta \sum_{j \in S_{2}} p_{j}^{*}}+1\right)+(T+2) \log (3) \\
& =2(T+2)\left(\sum_{i \in S_{2}} p_{i}^{*}\right) \log \left(2 \frac{T}{\Delta}+\sum_{j \in S_{2}} p_{j}^{*}\right) \\
& \quad-2(T+2)\left(\sum_{i \in S_{2}} p_{i}^{*}\right) \log \left(\sum_{j \in S_{2}} p_{j}^{*}\right)+(T+2) \log (3)
\end{aligned}
$$




$$
\begin{aligned}
& \stackrel{(\text { iii) }}{\leq} 2(T+2)\left(\sum_{i \in S_{2}} p_{i}^{*}\right) \log \left(2 \frac{T}{\Delta}+1\right)+2(T+2) \frac{\log (e)}{e}+(T+2) \log (3) \\
& \stackrel{(\text { iv })}{\leq} 2(T+2)\left(\frac{T}{\rho_{*}^{4} \Delta}\right) \log \left(2 \frac{T}{\Delta}+1\right)+2(T+2) \frac{\log (e)}{e}+(T+2) \log (3) \\
& \stackrel{(v)}{=} 2(T+2)\left(\frac{2 T(T+1)}{\rho_{*}^{2}}\right) \log \left(4 T(T+1) \rho_{*}^{2}+1\right) \\
& \quad+2(T+2) \frac{\log (e)}{e}+(T+2) \log (3) \\
& \stackrel{(v i)}{\leq} 2(T+2)(2 T(T+1))(4 T(T+1)+1) \frac{\log (e)}{e} \\
& \quad+2(T+2) \frac{\log (e)}{e}+(T+2) \log (3) \\
& =r_{1}(T) \text { independent of SNR, }
\end{aligned}
$$

where $(i)$ is due to Jensen's inequality, $(i i)$ is due to the power constraint $\sum_{i \in S_{2}} p_{i}^{*} l_{M i}^{*} \Delta \leq$ $T \Rightarrow \sum_{i \in S_{2}} p_{i}^{*} l_{M i}^{*} \leq \frac{T}{\Delta},(i i i)$ is due to the fact $0 \leq\left(\sum_{i \in S_{2}} p_{i}^{*}\right) \leq 1$ and $-x \log (x) \leq \frac{\log (e)}{e}$ for $x \in[0,1],(i v)$ is due to the fact $\sum_{i \in S_{2}} p_{i}^{*} l_{M i}^{*} \Delta \leq T$ (power constraint) and $\rho_{*}^{4} \Delta<l_{M i}^{*} \Delta$ in $S_{2}$, hence $\sum_{i \in S_{2}} p_{i}^{*} \rho_{*}^{4} \Delta \leq T$ and $\sum_{i \in S_{2}} p_{i}^{*} \leq \frac{T}{\rho_{*}^{4} \Delta},(v)$ is using $\Delta=\frac{1}{2(T+1) \rho_{*}^{2}}$ and $(v i)$ is due to the fact $\frac{1}{x} \log (x) \leq \frac{\log (e)}{e}$ for $x \in\left[1,+\infty\right.$ ) and assuming $\rho_{*}^{2}>1$. (If $\rho_{*}^{2} \leq 1$, then the system has zero $\mathrm{gDoF}$, so we consider only $\rho_{*}^{2}>1$ ). Hence it follows that

$$
\left|\left(\mathcal{P}_{2}\right)-\sum_{i \in S_{1}} p_{i}^{*} f\left(l_{1 i}^{*} \Delta, l_{2 i}^{*} \Delta, l_{3 i}^{*} \Delta\right)\right|=\left|\sum_{i \in S_{2}} p_{i}^{*} f\left(l_{1 i}^{*} \Delta, l_{2 i}^{*} \Delta, l_{3 i}^{*} \Delta\right)\right| \leq r_{1}(T) .
$$

Hence it follows that

$$
\mathcal{P}_{3}:\left\{\begin{array}{l}
\underset{\mathbb{E}\left[|a|^{2}+|b|^{2}+|c|^{2}\right] \leq T}{\operatorname{maximize}} \mathbb{E}\left[f\left(|a|^{2},|b|^{2},|c|^{2}\right)\right] \\
\text { Support }\left(|a|^{2},|b|^{2},|c|^{2}\right)=S_{1}
\end{array}\right.
$$

achieves the same gDoF as $\mathcal{P}_{2}$, because any non-zero probability outside $S_{1}$ in $\mathcal{P}_{2}$ can be assigned to $(0,0,0)$ in $\mathcal{P}_{3}$ by changing the value of the objective function by a constant independent of SNR. Hence

$$
\operatorname{gDoF}\left(\mathcal{P}_{1}\right)=\operatorname{gDoF}\left(\mathcal{P}_{2}\right)=\operatorname{gDoF}\left(\mathcal{P}_{3}\right)
$$

Now $\mathcal{P}_{3}$ is a linear program with a finite number of variables and constraints. (Also $\mathcal{P}_{3}$ has a finite optimum value because of Jensen's inequality.) The variables are $\left\{p_{i}\right\}_{i \in S_{1}}$ and the maximum number of nontrivial active constraints on $\left\{p_{i}\right\}_{i \in S_{1}}$ is two, derived from

$$
\mathbb{E}\left[|a|^{2}+|b|^{2}+|c|^{2}\right]=T, \quad \sum p_{i}=1
$$


Trivial constraints are $p_{i} \geq 0, i \in S_{1}$. Hence by the theory of linear optimization, there exists an optimal $\left\{p_{i}^{*}\right\}_{i \in S_{1}}$ with at most two nonzero values. Hence it follows that

$$
\mathcal{P}_{4}:\left\{\begin{array}{l}
\operatorname{maximize} \sum_{i=1}^{2} p_{i} f_{1}\left(\left|a_{i}\right|^{2},\left|b_{i}\right|^{2},\left|c_{i}\right|^{2}\right) \\
\sum_{i=1}^{2} p_{i}\left(\left|a_{i}\right|^{2}+\left|b_{i}\right|^{2}+\left|c_{i}\right|^{2}\right) \leq T, \\
\sum p_{i}=1, \\
\left|a_{i}\right|^{2},\left|b_{i}\right|^{2},\left|c_{i}\right|^{2} \geq 0
\end{array}\right.
$$

has $\left(\mathcal{P}_{4}\right) \geq\left(\mathcal{P}_{3}\right)$. Note that we have allowed $\left(\left|a_{i}\right|^{2},\left|b_{i}\right|^{2},\left|c_{i}\right|^{2}\right)_{i=1}^{2}$ to be real positive variables to be optimized. However, it is also clear that $\left(\mathcal{P}_{4}\right) \leq\left(\mathcal{P}_{1}\right)$. Hence

$$
\operatorname{gDoF}\left(\mathcal{P}_{1}\right)=\operatorname{gDoF}\left(\mathcal{P}_{2}\right)=\operatorname{gDoF}\left(\mathcal{P}_{3}\right)=\operatorname{gDoF}\left(\mathcal{P}_{4}\right)
$$

Now consider

$$
\mathcal{P}_{5}:\left\{\begin{array}{l}
\text { maximize } \sum_{i=1}^{2} p_{i} f_{1}\left(\left|a_{i}\right|^{2},\left|b_{i}\right|^{2},\left|c_{i}\right|^{2}\right) \\
p_{i}\left|a_{i}\right|^{2} \leq T, p_{i}\left|b_{i}\right|^{2} \leq T, p_{i}\left|c_{i}\right|^{2} \leq T, \\
\sum p_{i}=1, \\
\left|a_{i}\right|^{2},\left|b_{i}\right|^{2},\left|c_{i}\right|^{2} \geq 0 .
\end{array}\right.
$$

It can be easily shown that $\operatorname{gDoF}\left(\mathcal{P}_{4}\right)=\operatorname{gDoF}\left(\mathcal{P}_{5}\right)$, we omit the proof.

Lemma 23. Adding the constraints $\left|a_{i}\right|^{2} \leq T,\left|b_{i}\right|^{2} \leq T,\left|c_{i}\right|^{2} \leq T$ does not change the gDoF of $\mathcal{P}_{5}$.

Proof: We have

$$
\begin{aligned}
& f\left(|a|^{2},|b|^{2},|c|^{2}\right) \\
& =\log \left(\left(|a|^{2} \rho_{11}^{2}+|b|^{2} \rho_{12}^{2}+1\right)\left(|a|^{2} \rho_{21}^{2}+|b|^{2} \rho_{22}^{2}+1\right)+\left(|c|^{2} \rho_{12}^{2}+1\right)\left(|c|^{2} \rho_{22}^{2}+1\right)\right) \\
& \quad+(T-1) \log \left(\left(|a|^{2} \rho_{11}^{2}+1\right)\left(|c|^{2} \rho_{22}^{2}+1\right)+|b|^{2}\left(\rho_{12}^{2}+\rho_{22}^{2}\right)+\left(|a|^{2} \rho_{21}^{2}+1\right)\left(|c|^{2} \rho_{12}^{2}+1\right)\right) \\
& \quad-\log \left(\left(1+|a|^{2} \rho_{11}^{2}\right)\left(1+|c|^{2} \rho_{12}^{2}\right)+|b|^{2} \rho_{12}^{2}\right) \\
& \quad-\log \left(\left(1+|a|^{2} \rho_{21}^{2}\right)\left(1+|c|^{2} \rho_{22}^{2}\right)+|b|^{2} \rho_{22}^{2}\right) .
\end{aligned}
$$

Suppose $\left|a_{i}\right|^{2}>T$ and consider

$$
t_{1}=p_{i} \log \left(\left(\left|a_{i}\right|^{2} \rho_{11}^{2}+\left|b_{i}\right|^{2} \rho_{12}^{2}+1\right)\left(\left|a_{i}\right|^{2} \rho_{21}^{2}+\left|b_{i}\right|^{2} \rho_{22}^{2}+1\right)+\left(\left|c_{i}\right|^{2} \rho_{12}^{2}+1\right)\left(\left|c_{i}\right|^{2} \rho_{22}^{2}+1\right)\right) .
$$


We will show that setting $\left|a_{i}\right|^{2}=T$ would change the value of $t_{1}$ only by a constant independent of SNR. The other terms have a similar structure and can be handled in a similar way. If $\left(\left|c_{i}\right|^{2} \rho_{12}^{2}+1\right)\left(\left|c_{i}\right|^{2} \rho_{22}^{2}+1\right)>\left(\left|a_{i}\right|^{2} \rho_{11}^{2}+\left|b_{i}\right|^{2} \rho_{12}^{2}+1\right)\left(\left|a_{i}\right|^{2} \rho_{21}^{2}+\left|b_{i}\right|^{2} \rho_{22}^{2}+1\right)$, then the claim is trivially true; we can replace $\left|a_{i}\right|^{2}>T$ with $\left|a_{i}\right|^{2}=T$ while changing the value of $t_{1}$ by only a constant. Otherwise

$$
\begin{aligned}
t_{1} & \doteq p_{i} \log \left(\left(\left|a_{i}\right|^{2} \rho_{11}^{2}+\left|b_{i}\right|^{2} \rho_{12}^{2}+1\right)\left(\left|a_{i}\right|^{2} \rho_{21}^{2}+\left|b_{i}\right|^{2} \rho_{22}^{2}+1\right)\right) \\
& =\underbrace{p_{i} \log \left(\left|a_{i}\right|^{2} \rho_{11}^{2}+\left|b_{i}\right|^{2} \rho_{12}^{2}+1\right)}_{t_{11}}+\underbrace{p_{i} \log \left(\left|a_{i}\right|^{2} \rho_{21}^{2}+\left|b_{i}\right|^{2} \rho_{22}^{2}+1\right)}_{t_{12}} .
\end{aligned}
$$

Now consider $t_{11}=p_{i} \log \left(\left|a_{i}\right|^{2} \rho_{11}^{2}+\left|b_{i}\right|^{2} \rho_{12}^{2}+1\right)$. If $\left|a_{i}\right|^{2} \rho_{11}^{2}<\left|b_{i}\right|^{2} \rho_{12}^{2}+1$, then we can replace $\left|a_{i}\right|^{2}>T$ with $\left|a_{i}\right|^{2}=T$ without losing $\mathrm{gDoF}$.

If $\left|a_{i}\right|^{2} \rho_{11}^{2}>\left|b_{i}\right|^{2} \rho_{12}^{2}+1$, then $t_{11} \doteq p_{i} \log \left(\left|a_{i}\right|^{2} \rho_{11}^{2}+1\right)$, where the approximation is tight within a constant (constant less than 1). Now if we replace $\left|a_{i}\right|^{2}>T$ with $\left|a_{i}\right|^{2}=T$, the difference arising is bounded independent of SNR, as seen below:

$$
\begin{aligned}
p_{i} \log \left(\left|a_{i}\right|^{2} \rho_{11}^{2}+1\right) & \stackrel{(i)}{\leq} p_{i} \log \left(\frac{T}{p_{i}} \rho_{11}^{2}+1\right) \\
& =p_{i} \log \left(T \rho_{11}^{2}+p_{i}\right)-p_{i} \log \left(p_{i}\right) \\
& \leq p_{i} \log \left(T \rho_{11}^{2}+1\right)-p_{i} \log \left(p_{i}\right)
\end{aligned}
$$

where $(i)$ is because $p_{i}\left|a_{i}\right|^{2} \leq T$ due to the power constraint. We also have $\left|a_{i}\right|^{2}>T$, hence it follows that

$$
\left|p_{i} \log \left(\left|a_{i}\right|^{2} \rho_{11}^{2}+1\right)-p_{i} \log \left(T \rho_{11}^{2}+1\right)\right| \leq\left|p_{i} \log \left(p_{i}\right)\right| \leq \frac{\log (e)}{e}
$$

Following the same logic for the other terms, it can be shown that adding the constraints $\left|a_{i}\right|^{2} \leq$ $T,\left|b_{i}\right|^{2} \leq T,\left|c_{i}\right|^{2} \leq T$ does not change the $\mathrm{gDoF}$ of $\mathcal{P}_{5}$.

With the additional constraints $\left|a_{i}\right|^{2} \leq T,\left|b_{i}\right|^{2} \leq T,\left|c_{i}\right|^{2} \leq T$, the existing constraints $p_{i}\left|a_{i}\right|^{2} \leq T, p_{i}\left|b_{i}\right|^{2} \leq T, p_{i}\left|c_{i}\right|^{2} \leq T$ become redundant. Hence we have $\operatorname{gDoF}\left(\mathcal{P}_{5}\right)=$ $\operatorname{gDoF}\left(\mathcal{P}_{6}\right)$ for $\mathcal{P}_{6}$ defined as

$$
\mathcal{P}_{6}:\left\{\begin{array}{l}
\operatorname{maximize} \sum_{i=1}^{2} p_{i} f\left(\left|a_{i}\right|^{2},\left|b_{i}\right|^{2},\left|c_{i}\right|^{2}\right) \\
\left|a_{i}\right|^{2} \leq T,\left|b_{i}\right|^{2} \leq T,\left|c_{i}\right|^{2} \leq T, \\
\sum p_{i}=1 .
\end{array}\right.
$$


It is clear from the structure of $\mathcal{P}_{6}$ that the solution has $\left(\left|a_{1}\right|^{2},\left|b_{1}\right|^{2},\left|c_{1}\right|^{2}\right)=\left(\left|a_{2}\right|^{2},\left|b_{2}\right|^{2},\left|c_{2}\right|^{2}\right)$. Hence it suffices to solve $\mathcal{P}_{7}$ defined as

$$
\mathcal{P}_{7}:\left\{\begin{array}{l}
\operatorname{maximize} f\left(|a|^{2},|b|^{2},|c|^{2}\right) \\
|a|^{2} \leq T,|b|^{2} \leq T,|c|^{2} \leq T
\end{array}\right.
$$

\section{REFERENCES}

[1] J. Sebastian, A. Sengupta, and S. N. Diggavi, "On capacity of noncoherent MIMO with asymmetric link strengths," in IEEE International Symposium on Information Theory, June 2017, pp. 541-545.

[2] T. L. Marzetta and B. M. Hochwald, "Capacity of a mobile multiple-antenna communication link in Rayleigh flat fading," IEEE Transactions on Information Theory, vol. 45, no. 1, pp. 139-157, 1999.

[3] L. Zheng and D. N. C. Tse, "Communication on the Grassmann manifold: a geometric approach to the noncoherent multiple-antenna channel," IEEE Transactions on Information Theory, vol. 48, no. 2, pp. 359-383, Feb 2002.

[4] W. Yang, G. Durisi, and E. Riegler, "On the capacity of large-MIMO block-fading channels," IEEE Journal on Selected Areas in Communications, vol. 31, no. 2, pp. 117-132, February 2013.

[5] G. Taricco and M. Elia, "Capacity of fading channel with no side information," Electronics Letters, vol. 33, no. 16, pp. 1368-1370, Jul 1997.

[6] I. C. Abou-Faycal, M. D. Trott, and S. Shamai, "The capacity of discrete-time memoryless Rayleigh-fading channels," IEEE Transactions on Information Theory, vol. 47, no. 4, pp. 1290-1301, May 2001.

[7] A. Lapidoth and S. M. Moser, "Capacity bounds via duality with applications to multiple-antenna systems on flat-fading channels," IEEE Transactions on Information Theory, vol. 49, no. 10, pp. 2426-2467, 2003.

[8] R. H. Etkin, D. N. C. Tse, and H. Wang, "Gaussian interference channel capacity to within one bit," IEEE Transactions on Information Theory, vol. 54, no. 12, pp. 5534-5562, 2008.

[9] S. A. Jafar and S. Vishwanath, "Generalized degrees of freedom of the symmetric gaussian $k$-user interference channel," IEEE Transactions on Information Theory, vol. 56, no. 7, pp. 3297-3303, July 2010.

[10] S. Karmakar and M. K. Varanasi, "The generalized degrees of freedom region of the mimo interference channel and its achievability," IEEE Transactions on Information Theory, vol. 58, no. 12, pp. 7188-7203, Dec 2012.

[11] V. I. Morgenshtern, E. Riegler, W. Yang, G. Durisi, S. Lin, B. Sturmfels, and H. Bolcskei, "Capacity pre-log of noncoherent SIMO channels via Hironaka's theorem," IEEE Transactions on Information Theory, vol. 59, no. 7, pp. 4213-4229, July 2013.

[12] G. Koliander, E. Riegler, G. Durisi, and F. Hlawatsch, "Degrees of freedom of generic block-fading MIMO channels without apriori channel state information," IEEE Transactions on Information Theory, vol. 60, no. 12, pp. 7760-7781, Dec 2014.

[13] A. Lapidoth, "On the high-SNR capacity of noncoherent networks," IEEE Transactions on Information Theory, vol. 51, no. 9, pp. 3025-3036, Sept 2005.

[14] T. Koch and G. Kramer, "On noncoherent fading relay channels at high signal-to-noise ratio," IEEE Transactions on Information Theory, vol. 59, no. 4, pp. 2221-2241, April 2013.

[15] R. H. Gohary and H. Yanikomeroglu, "Grassmannian signalling achieves tight bounds on the ergodic high-SNR capacity of the noncoherent MIMO full-duplex relay channel," IEEE Transactions on Information Theory, vol. 60, no. 5, pp. 2480-2494, May 2014. 
[16] N. Bhushan, J. Li, D. Malladi, R. Gilmore, D. Brenner, A. Damnjanovic, R. Sukhavasi, C. Patel, and S. Geirhofer, "Network densification: the dominant theme for wireless evolution into 5G," IEEE Communications Magazine, vol. 52, no. 2, pp. 82-89, 2014.

[17] J. Wu, Z. Zhang, Y. Hong, and Y. Wen, “Cloud radio access network (C-RAN): a primer,” IEEE Network, vol. 29, no. 1, pp. $35-41,2015$.

[18] R. Irmer, H. Droste, P. Marsch, M. Grieger, G. Fettweis, S. Brueck, H. Mayer, L. Thiele, and V. Jungnickel, "Coordinated multipoint: Concepts, performance, and field trial results," IEEE Communications Magazine, vol. 49, no. 2, pp. 102-111, February 2011.

[19] C. Karakus and S. N. Diggavi, "Enhancing multiuser MIMO through opportunistic D2D cooperation," IEEE Transactions on Wireless Communications, vol. 16, no. 9, pp. 5616-5629, Sept 2017.

[20] M. Cardone, D. Tuninetti, R. Knopp, and U. Salim, "Gaussian half-duplex relay networks: Improved constant gap and connections with the assignment problem," IEEE Transactions on Information Theory, vol. 60, no. 6, pp. 3559-3575, Jun 2014.

[21] J. Sebastian, C. Karakus, S. N. Diggavi, and I. H. Wang, "Rate splitting is approximately optimal for fading Gaussian interference channels," in Annual Allerton Conference on Communication, Control, and Computing, Sept 2015, pp. 315321.

[22] M. Abramowitz and I. A. Stegun, Handbook of mathematical functions: with formulas, graphs, and mathematical tables. Courier Corporation, 1964, no. 55.

[23] T. Cover and J. Thomas, Elements of Information Theory. Wiley, 2012. [Online]. Available: https://books.google.com/ books?id=VWq5GG6ycxMC 


\section{ApPENDIX C}

\section{Proof of THEOREM 2; DECOMPOSING INTO DISJOINT PARTS OF MIMO CHANNELS}

Here we prove that for a MIMO system whose channel can be decomposed into disjoint parts, the capacity can be achieved by allocating power to the disjoint parts separately. Let the channel matrix $G$ of the system be block diagonal as $G=\operatorname{diag}\left(G_{1}, \ldots, G_{K}\right)$, where $G_{i}$ are the diagonal blocks corresponding to the disjoint parts of the channel, then the capacity $C\left(P, \operatorname{diag}\left(G_{1}, \ldots, G_{K}\right)\right)$ of the channel for a power $P$ can be achieved by splitting power across the blocks, i.e., $C\left(P, \operatorname{diag}\left(G_{1}, \ldots, G_{K}\right)\right)=\max _{P_{1}+\cdots+P_{K} \leq P}\left(C\left(P_{1}, G_{1}\right)+\cdots+C\left(P_{K}, G_{K}\right)\right)$. We just need to show that for $G=\operatorname{diag}\left(G_{1}, G_{2}\right)$, the capacity of the channel can be achieved by a power splitting across the two blocks of channels $G_{1}, G_{2}$ i.e.,

$$
C\left(P, \operatorname{diag}\left(G_{1}, G_{2}\right)\right)=\max _{P_{1}+P_{2} \leq P}\left(C\left(P_{1}, G_{1}\right)+C\left(P_{2}, G_{2}\right)\right)
$$

and the general result for multiple disjoint parts in the MIMO channel will follow due to induction. We have

$$
\begin{aligned}
h(Y) & \stackrel{(i i)}{\leq} h\left(Y_{G 1}\right)+h\left(Y_{G 2}\right) \\
h(Y \mid X) & =h\left(Y_{G 1} Y_{G 2} \mid X_{G 1} X_{G 2}\right) \\
& =h\left(Y_{G 1} \mid X_{G 1} X_{G 2}\right)+h\left(Y_{G 2} \mid Y_{G 1} X_{G 1} X_{G 2}\right) \\
& \stackrel{(i i)}{=} h\left(Y_{G 1} \mid X_{G 2}\right)+h\left(Y_{G 2} \mid X_{G 2}\right),
\end{aligned}
$$

where $(i)$ is because conditioning reduces entropy and $(i i)$ is because $X_{G 2}-X_{G 1}-Y_{G 1}$ and $\left(X_{G 1}, Y_{G 1}\right)-X_{G 2}-Y_{G 2}$ are Markov chains.

Hence

$$
I(X ; Y) \leq I\left(X_{G 1} ; Y_{G 1}\right)+I\left(X_{G 2} ; Y_{G 2}\right)
$$

subject to $\mathbb{E}\left[\left\|X_{G 1}\right\|^{2}+\left\|X_{G 2}\right\|^{2}\right] \leq P$. The RHS can be achieved by treating the two blocks of channels $G_{1}, G_{2}$ separately with a power allocation, hence

$$
C\left(P, \operatorname{diag}\left(G_{1}, G_{2}\right)\right)=\max _{P_{1}+P_{2} \leq P}\left(C\left(P_{1}, G_{1}\right)+C\left(P_{2}, G_{2}\right)\right) .
$$




\section{APPENDIX D}

INNER BOUND FOR THE $2 \times 2$ MIMO CHANNEL

Here we prove the achievability result from Theorem 7 for the $2 \times 2$ MIMO channel with exponents $\gamma_{D}$ in the direct links and $\gamma_{C L}$ in the crosslinks. We use the input distribution

$$
X=\left[\begin{array}{cccccc}
a & 0 & 0 & . & . & 0 \\
\eta & c & 0 & . & . & 0
\end{array}\right] Q
$$

with constants $a, c$ and $\eta \sim \mathcal{C N}\left(0,|b|^{2}\right)$ with

$$
|a|^{2}=\mathrm{SNR}^{\gamma_{a}},|b|^{2}=\mathrm{SNR}^{\gamma_{b}},|c|^{2}=\mathrm{SNR}^{\gamma_{c}}, \gamma_{a} \leq 0, \gamma_{b} \leq 0, \gamma_{c} \leq 0
$$

With this choice, we proceed to lower bound $I(X ; Y)$.

$$
\begin{aligned}
I(X ; Y) & =h(Y)-h(Y \mid X) \\
h(Y) & =h(G X+W) \\
& \geq h(G X) \\
& =h\left(\left[\begin{array}{ll}
g_{11} & g_{12} \\
g_{21} & g_{22}
\end{array}\right]\left[\begin{array}{llllll}
a & 0 & 0 & . & . & 0 \\
\eta & c & 0 & . & . & 0
\end{array}\right] Q\right) .
\end{aligned}
$$

Now

$$
\begin{aligned}
& {\left[\begin{array}{ll}
g_{11} & g_{12} \\
g_{21} & g_{22}
\end{array}\right]\left[\begin{array}{llllll}
a & 0 & 0 & \cdot & \cdot & 0 \\
\eta & c & 0 & \cdot & \cdot & 0
\end{array}\right]}
\end{aligned}
$$

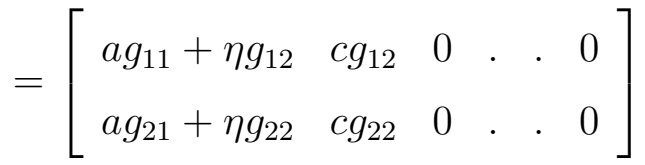

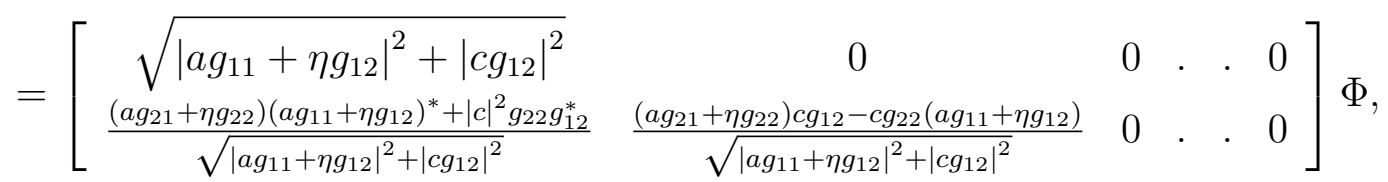

where in the last step, we performed an LQ transformation and $\Phi$ is unitary. Hence due to the property of isotropic unitary matrices and steps similar to (51) to (54) in Section IV-D, we get:

$h(G X)$

$\stackrel{(i)}{=} h\left(\sqrt{\left|a g_{11}+\eta g_{12}\right|^{2}+\left|c g_{12}\right|^{2}} \bar{q}_{1}^{(T)}\right)$ 


$$
\begin{aligned}
& +h\left(\left[\frac{\left(a g_{21}+\eta g_{22}\right)\left(a g_{11}+\eta g_{12}\right)^{*}+|c|^{2} g_{22} g_{12}^{*}}{\sqrt{\left|a g_{11}+\eta g_{12}\right|^{2}+\left|c g_{12}\right|^{2}}}, \frac{a c\left(g_{12} g_{21}-g_{11} g_{22}\right)}{\sqrt{\left|a g_{11}+\eta g_{12}\right|^{2}+\left|c g_{12}\right|^{2}}} \bar{q}_{2}^{(T-1)}\right] \mid \xi_{11}\right) \\
& \stackrel{(i i)}{=} h\left(\sqrt{\left|a g_{11}+\eta g_{12}\right|^{2}+\left|c g_{12}\right|^{2}} \bar{q}_{1}^{(T)}\right)-T \mathbb{E}\left[\log \left(\left|a g_{11}+\eta g_{12}\right|^{2}+\left|c g_{12}\right|^{2}\right)\right] \\
& +\underbrace{h\left(\left[\left(a g_{21}+\eta g_{22}\right)\left(a g_{11}+\eta g_{12}\right)^{*}+|c|^{2} g_{22} g_{12}^{*}, a c\left(g_{12} g_{21}-g_{11} g_{22}\right) \bar{q}_{2}^{(T-1)}\right] \mid \xi_{11}\right)}_{\alpha} \text {, }
\end{aligned}
$$

where $\bar{q}_{1}^{(i)}$ denotes an $i$ dimensional isotropically distributed random unit vector and $\xi_{11}=$ $\sqrt{\left|a g_{11}+\eta g_{12}\right|^{2}+\left|c g_{12}\right|^{2}}$. The step $(i)$ involved the simplification $\left(a g_{21}+\eta g_{22}\right) c g_{12}-c g_{22}\left(a g_{11}+\eta g_{12}\right)=$ $a c\left(g_{12} g_{21}-g_{11} g_{22}\right)$ and the step $(i)$ involved moving $\sqrt{\left|a g_{11}+\eta g_{12}\right|^{2}+\left|c g_{12}\right|^{2}}$ from the denominator. Now

$\alpha$

$$
\begin{aligned}
& =h\left(a c\left(g_{12} g_{21}-g_{11} g_{22}\right) \bar{q}_{2}^{(T-1)} \mid \xi_{11}\right) \\
& +h\left(\left(a g_{21}+\eta g_{22}\right)\left(a g_{11}+\eta g_{12}\right)^{*}+|c|^{2} g_{22} g_{12}^{*} \mid a c\left(g_{12} g_{21}-g_{11} g_{22}\right) \bar{q}_{2}^{(T-1)}, \xi_{11}\right) \\
& \stackrel{(i)}{\geq}+h\left(\left|a c\left(g_{12} g_{21}-g_{11} g_{22}\right)\right|^{2} \mid \xi_{11}\right)+(T-2) \mathbb{E}\left[\log \left(\left|a c\left(g_{12} g_{21}-g_{11} g_{22}\right)\right|^{2}\right)\right]+\log \left(\frac{\pi^{T-1}}{\Gamma(T-1)}\right) \\
& +h\left(\left(a g_{21}+\eta g_{22}\right)\left(a g_{11}+\eta g_{12}\right)^{*}+|c|^{2} g_{22} g_{12}^{*} \mid a c\left(g_{12} g_{21}-g_{11} g_{22}\right), \bar{q}_{2}^{(T-1)}, \xi_{11}\right) \\
& \stackrel{(i i)}{=} h\left(a c\left(g_{12} g_{21}-g_{11} g_{22}\right) \mid \xi_{11}\right)-\log (\pi)+(T-2) \mathbb{E}\left[\log \left(\left|a c\left(g_{12} g_{21}-g_{11} g_{22}\right)\right|^{2}\right)\right] \\
& +\log \left(\frac{\pi^{T-1}}{\Gamma(T-1)}\right)+h\left(\left(a g_{21}+\eta g_{22}\right)\left(a g_{11}+\eta g_{12}\right)^{*}+|c|^{2} g_{22} g_{12}^{*} \mid a c\left(g_{12} g_{21}-g_{11} g_{22}\right), \xi_{11}\right) \\
& \stackrel{(i i i)}{=}(T-2) \mathbb{E}\left[\log \left(\left|a c g_{12} g_{21}-a c g_{11} g_{22}\right|^{2}\right)\right]+\log \left(\frac{\pi^{T-2}}{\Gamma(T-1)}\right)+2 \mathbb{E}\left[\log \left(\left|a g_{11}+\eta g_{12}\right|^{2}+\left|c g_{12}\right|^{2}\right)\right] \\
& h\left(\left[\frac{\left(a g_{21}+\eta g_{22}\right)\left(a g_{11}+\eta g_{12}\right)^{*}+|c|^{2} g_{22} g_{12}^{*}}{\sqrt{\left|a g_{11}+\eta g_{12}\right|^{2}+\left|c g_{12}\right|^{2}}}, \frac{a c\left(g_{12} g_{21}-g_{11} g_{22}\right)}{\sqrt{\left|a g_{11}+\eta g_{12}\right|^{2}+\left|c g_{12}\right|^{2}}}\right] \mid \xi_{11}\right) \\
& \stackrel{(i v)}{\geq}(T-2) \mathbb{E}\left[\log \left(\left|a c g_{12} g_{21}-a c g_{11} g_{22}\right|^{2}\right)\right]+\log \left(\frac{\pi^{T-2}}{\Gamma(T-1)}\right)+2 \mathbb{E}\left[\log \left(\left|a g_{11}+\eta g_{12}\right|^{2}+\left|c g_{12}\right|^{2}\right)\right] \\
& h\left(\left[\frac{\left(a g_{21}+\eta g_{22}\right)\left(a g_{11}+\eta g_{12}\right)^{*}+|c|^{2} g_{22} g_{12}^{*}}{\sqrt{\left|a g_{11}+\eta g_{12}\right|^{2}+\left|c g_{12}\right|^{2}}}, \frac{\left(a g_{21}+\eta g_{22}\right) c g_{12}-c g_{22}\left(a g_{11}+\eta g_{12}\right)}{\sqrt{\left|a g_{11}+\eta g_{12}\right|^{2}+\left|c g_{12}\right|^{2}}}\right] \mid a g_{11}+\eta g_{12}, g_{12}\right) \\
& \stackrel{(v)}{=}(T-2) \mathbb{E}\left[\log \left(\left|a c g_{12} g_{21}-a c g_{11} g_{22}\right|^{2}\right)\right]+\log \left(\frac{\pi^{T-2}}{\Gamma(T-1)}\right)+2 \mathbb{E}\left[\log \left(\left|a g_{11}+\eta g_{12}\right|^{2}+\left|c g_{12}\right|^{2}\right)\right] \\
& +h\left(\left[a g_{21}+\eta g_{22} \quad c g_{22}\right] \mid a g_{11}+\eta g_{12}, g_{12}\right),
\end{aligned}
$$

where $(i)$ is using Lemma 13 on page 18 for the first term and the fact that conditioning reduces entropy for the second term, (ii) is using Lemma 13 on $h\left(a c\left(g_{12} g_{21}-g_{11} g_{22}\right) \mid \xi_{11}\right)$. Note that 
with $\theta \sim \operatorname{Unif}[0,2 \pi]$ independent of other random variables, $a c\left(g_{12} g_{21}-g_{11} g_{22}\right) e^{i \theta} \mid \xi_{11}$ and $a c\left(g_{12} g_{21}-g_{11} g_{22}\right) \mid \xi_{11}$ have the same distribution; $e^{i \theta}$ is the unitary distribution in one dimension; hence Lemma 13 can be applied. The step $(i i i)$ is using $\xi_{11}=\sqrt{\left|a g_{11}+\eta g_{12}\right|^{2}+\left|c g_{12}\right|^{2}}$ and rearranging the terms, $(i v)$ is because conditioning reduces entropy and $(v)$ is by a unitary transformation on the last term. Hence by substituting (148) in (147), we have

$$
\begin{aligned}
& h(G X) \\
& \geq h\left(\sqrt{\left|a g_{11}+\eta g_{12}\right|^{2}+\left|c g_{12}\right|^{2}}{\overline{q_{1}}}^{(T)}\right)-(T-2) \mathbb{E}\left[\log \left(\left|a g_{11}+\eta g_{12}\right|^{2}+\left|c g_{12}\right|^{2}\right)\right] \\
& +\log \left(\frac{\pi^{T-2}}{\Gamma(T-1)}\right)+(T-2) \mathbb{E}\left[\log \left(\left|a c g_{12} g_{21}-a c g_{11} g_{22}\right|^{2}\right)\right] \\
& +h\left(\left[a g_{21}+\eta g_{22} \quad c g_{22}\right] \mid a g_{11}+\eta g_{12}, g_{12}\right) \\
& \stackrel{(i)}{=} h\left(\left|a g_{11}+\eta g_{12}\right|^{2}+\left|c g_{12}\right|^{2}\right)+\mathbb{E}\left[\log \left(\left|a g_{11}+\eta g_{12}\right|^{2}+\left|c g_{12}\right|^{2}\right)\right]+\log \left(\frac{\pi^{T-2}}{\Gamma(T-1)}\right) \\
& +\log \left(\frac{\pi^{T}}{\Gamma(T)}\right)+(T-2) \mathbb{E}\left[\log \left(\left|a c g_{12} g_{21}-a c g_{11} g_{22}\right|^{2}\right)\right] \\
& +h\left(\left[a g_{21}+\eta g_{22} \quad c g_{22}\right] \mid a g_{11}+\eta g_{12}, g_{12}\right) \text {, }
\end{aligned}
$$

where $(i)$ is using Lemma 13 on $h\left(\sqrt{\left|a g_{11}+\eta g_{12}\right|^{2}+\left|c g_{12}\right|^{2}} \overline{q_{1}}(T)\right)$. Also

$h\left(\left[a g_{21}+\eta g_{22} \quad c g_{22}\right] \mid a g_{11}+\eta g_{12}, g_{12}\right)=h\left(c g_{22}\right)+h\left(a g_{21}+\eta g_{22} \mid a g_{11}+\eta g_{12}, g_{12}, g_{22}\right)$.

Using $\eta \sim \mathcal{C N}\left(0,|b|^{2}\right)$, we have

$$
\begin{aligned}
& h\left(a g_{21}+\eta g_{22} \mid a g_{11}+\eta g_{12}, g_{12}, g_{22}\right) \\
& =h\left(a g_{21}+\eta g_{22}, a g_{11}+\eta g_{12} \mid g_{12}, g_{22}\right)-h\left(a g_{11}+\eta g_{12} \mid g_{12}, g_{22}\right) \\
& =\mathbb{E}\left[\log \left(\left|\begin{array}{cc}
|a|^{2} \rho_{21}^{2}+|b|^{2}\left|g_{22}\right|^{2} & |b|^{2} g_{22} g_{12}^{*} \\
|b|^{2} g_{22}^{*} g_{12} & |a|^{2} \rho_{11}^{2}+|b|^{2}\left|g_{12}\right|^{2}
\end{array}\right|\right)\right] \\
& -\mathbb{E}\left[\log \left(|a|^{2} \rho_{11}^{2}+|b|^{2}\left|g_{12}\right|^{2}\right)\right]+\log (\pi e) \\
& \stackrel{(i)}{\geq} \log \left(|a|^{4} \mathrm{SNR}^{\gamma_{11}+\gamma_{21}}+|a|^{2}|b|^{2} \mathrm{SNR}^{\gamma_{12}+\gamma_{21}}+|a|^{2}|b|^{2} \mathrm{SNR}^{\gamma_{11}+\gamma_{22}}\right) \\
& -\log \left(|a|^{2} \operatorname{SNR}^{\gamma_{11}}+|b|^{2} \operatorname{SNR}^{\gamma_{12}}\right)+\log (\pi e)-2 \gamma_{E} \log (e),
\end{aligned}
$$


where $(i)$ is using Lemma 9 from page 16 on $\left|g_{22}\right|^{2}$ and $\left|g_{12}\right|^{2}$. Now substituting (154) and 151) in 150 we get

$$
\begin{aligned}
h(G X) \geq h & \left(\left|a g_{11}+\eta g_{12}\right|^{2}+\left|c g_{12}\right|^{2}\right)+\mathbb{E}\left[\log \left(\left|a g_{11}+\eta g_{12}\right|^{2}+\left|c g_{12}\right|^{2}\right)\right]+\log \left(\frac{\pi^{T-2}}{\Gamma(T-1)}\right) \\
& +\log \left(\frac{\pi^{T}}{\Gamma(T)}\right)+(T-2) \mathbb{E}\left[\log \left(\left|a c g_{12} g_{21}-a c g_{11} g_{22}\right|^{2}\right)\right]+h\left(c g_{22}\right) \\
& +\log \left(|a|^{4} \mathrm{SNR}^{\gamma_{11}+\gamma_{21}}+|a|^{2}|b|^{2} \mathrm{SNR}^{\gamma_{12}+\gamma_{21}}+|a|^{2}|b|^{2} \mathrm{SNR}^{\gamma_{11}+\gamma_{22}}\right) \\
& -\log \left(|a|^{2} \mathrm{SNR}^{\gamma_{11}}+|b|^{2} \operatorname{SNR}^{\gamma_{12}}\right)+\log (\pi e)-2 \gamma_{E} \log (e) .
\end{aligned}
$$

Now we use our choice $\eta \sim \mathcal{C N}\left(0,|b|^{2}\right),|a|^{2}=\mathrm{SNR}^{-\gamma_{a}},|b|^{2}=\mathrm{SNR}^{-\gamma_{b}},|c|^{2}=\mathrm{SNR}^{-\gamma_{c}}, \gamma_{a} \geq$ $0, \gamma_{b} \geq 0, \gamma_{c} \geq 0$. We then have

$$
\begin{aligned}
& h\left(\left|a g_{11}+\eta g_{12}\right|^{2}+\left|c g_{12}\right|^{2}\right) \\
& \stackrel{(i)}{\geq} \max \left(h\left(\left|a g_{11}+\eta g_{12}\right|^{2}\right), h\left(\left|c g_{12}\right|^{2}\right)\right) \\
& \geq \max \left(h\left(\left|a g_{11}+\eta g_{12}\right|^{2} \mid g_{12}\right), h\left(\left|c g_{12}\right|^{2}\right)\right) \\
& \stackrel{(i i)}{=} \max \left(\mathbb{E}\left[\log \left(\mathrm{SNR}^{-\gamma_{a}+\gamma_{11}}+\mathrm{SNR}^{-\gamma_{b}}\left|g_{12}\right|^{2}\right)\right], \log \left(\mathrm{SNR}^{-\gamma_{c}+\gamma_{12}}\right)\right) \\
& \doteq \max \left(\log \left(\mathrm{SNR}^{-\gamma_{a}+\gamma_{11}}+\mathrm{SNR}^{-\gamma_{b}+\gamma_{12}}\right), \log \left(\mathrm{SNR}^{-\gamma_{c}+\gamma_{12}}\right)\right),
\end{aligned}
$$

where $(i)$ is using the fact that conditioning reduces entropy, $(i i)$ is using the property of exponential distributions and (iii) is using Lemma 9. Hence

$$
\lim _{\mathrm{SNR} \rightarrow \infty} h\left(\left|a g_{11}+\eta g_{12}\right|^{2}+\left|c g_{12}\right|^{2}\right) / \log (\mathrm{SNR}) \geq \max \left(-\gamma_{a}+\gamma_{11},-\gamma_{b}+\gamma_{12},-\gamma_{c}+\gamma_{12}\right) .
$$

Now

$$
\begin{aligned}
& \mathbb{E}\left[\log \left(\left|a g_{11}+\eta g_{12}\right|^{2}+\left|c g_{12}\right|^{2}\right)\right] \\
& \geq \max \left(\mathbb{E}\left[\log \left(\left|a g_{11}+\eta g_{12}\right|^{2}\right)\right], \mathbb{E}\left[\log \left(\left|c g_{12}\right|^{2}\right)\right]\right) \\
& \stackrel{(i)}{=} \max \left(\log \left(\mathrm{SNR}^{-\gamma_{a}+\gamma_{11}}+\mathrm{SNR}^{\gamma_{b}+\gamma_{12}}\right), \log \left(\mathrm{SNR}^{-\gamma_{c}+\gamma_{12}}\right)\right),
\end{aligned}
$$

where $(i)$ is using Lemma 9 . Also

$$
\mathbb{E}\left[\log \left(\left|a c g_{12} g_{21}-a c g_{11} g_{22}\right|^{2}\right)\right] \doteq \log \left(\mathrm{SNR}^{-\gamma_{a}-\gamma_{c}+\gamma_{12}+\gamma_{21}}+\mathrm{SNR}^{-\gamma_{a}-\gamma_{c}+\gamma_{11}+\gamma_{22}}\right)
$$

using Lemma 9 repeatedly. Similarly evaluating other terms in 155, we get

$$
\lim _{\mathrm{SNR} \rightarrow \infty} \frac{h(G X)}{\log (\mathrm{SNR})}
$$




$$
\begin{aligned}
\geq & 2 \max \left(-\gamma_{a}+\gamma_{11},-\gamma_{b}+\gamma_{12},-\gamma_{c}+\gamma_{12}\right) \\
& +(T-2)\left(-\gamma_{a}-\gamma_{c}+\max \left(\gamma_{12}+\gamma_{21}, \gamma_{11}+\gamma_{22}\right)\right)-\gamma_{c}+\gamma_{22} \\
& +\max \left(-2 \gamma_{a}+\gamma_{11}+\gamma_{21},-\gamma_{a}-\gamma_{b}+\gamma_{12}+\gamma_{21},-\gamma_{a}-\gamma_{b}+\gamma_{11}+\gamma_{22}\right) \\
& -\max \left(-\gamma_{a}+\gamma_{11},-\gamma_{b}+\gamma_{12}\right) .
\end{aligned}
$$

Also, using (24), (28) we have

$$
\begin{aligned}
& h(Y \mid X) \\
&= \mathbb{E}\left[\log \left(|a|^{2} \rho_{11}^{2}+|\eta|^{2} \rho_{12}^{2}+|c|^{2} \rho_{12}^{2}+|a|^{2}|c|^{2} \rho_{11}^{2} \rho_{12}^{2}+1\right)\right] \\
&+\mathbb{E}\left[\log \left(|a|^{2} \rho_{21}^{2}+|\eta|^{2} \rho_{22}^{2}+|c|^{2} \rho_{22}^{2}+|a|^{2}|c|^{2} \rho_{21}^{2} \rho_{22}^{2}+1\right)\right] \\
&+2 T \log (\pi e) .
\end{aligned}
$$

Now since $\eta \sim \mathcal{C N}\left(0,|b|^{2}\right)$ and $|a|^{2}=\mathrm{SNR}^{-\gamma_{a}},|b|^{2}=\mathrm{SNR}^{-\gamma_{b}},|c|^{2}=\mathrm{SNR}^{-\gamma_{c}}$ and Lemma 9 we get

$$
\begin{aligned}
& h(Y \mid X) \\
& \doteq \log \left(|a|^{2} \rho_{11}^{2}+|b|^{2} \rho_{12}^{2}+|c|^{2} \rho_{12}^{2}+|a|^{2}|c|^{2} \rho_{11}^{2} \rho_{12}^{2}+1\right) \\
& \quad+\log \left(|a|^{2} \rho_{21}^{2}+|b|^{2} \rho_{22}^{2}+|c|^{2} \rho_{22}^{2}+|a|^{2}|c|^{2} \rho_{21}^{2} \rho_{22}^{2}+1\right)
\end{aligned}
$$

and hence

$$
\begin{aligned}
& \lim _{\operatorname{SNR} \rightarrow \infty} \frac{h(Y \mid X)}{\log (\mathrm{SNR})} \\
& \doteq \max \left(-\gamma_{a}+\gamma_{11},-\gamma_{b}+\gamma_{12},-\gamma_{c}+\gamma_{12},-\gamma_{a}-\gamma_{c}+\gamma_{11}+\gamma_{12}, 0\right) \\
& \quad+\max \left(-\gamma_{a}+\gamma_{21},-\gamma_{b}+\gamma_{22},-\gamma_{c}+\gamma_{22},-\gamma_{a}-\gamma_{c}+\gamma_{21}+\gamma_{22}, 0\right)
\end{aligned}
$$

Using (163), 166) with $\gamma_{a}=0, \gamma_{c}=0, \gamma_{b}=0, \gamma_{11}=\gamma_{22}=\gamma_{D}>\gamma_{C L}=\gamma_{12}=\gamma_{21}$ we get

$$
\lim _{\mathrm{SNR} \rightarrow \infty} \frac{h(G X)}{\log (\mathrm{SNR})} \geq 2 T \gamma_{D}
$$

and

$$
\lim _{\mathrm{SNR} \rightarrow \infty} \frac{h(Y \mid X)}{\log (\mathrm{SNR})}=2\left(\gamma_{D}+\gamma_{C L}\right)
$$

Hence we have

$$
\lim _{\mathrm{SNR} \rightarrow \infty} \frac{1}{T} \frac{I(X ; Y)}{\log (\mathrm{SNR})} \geq 2\left(\left(1-\frac{1}{T}\right) \gamma_{D}-\frac{1}{T} \gamma_{C L}\right)
$$


achievable. Also with $\gamma_{a}=0, \gamma_{c}=\gamma_{C L}, \gamma_{b}=0, \gamma_{11}=\gamma_{22}=\gamma_{D}>\gamma_{C L}=\gamma_{12}=\gamma_{21}$ in (163), (166) we get

$$
\lim _{\mathrm{SNR} \rightarrow \infty} \frac{h(G X)}{\log (\mathrm{SNR})} \geq 2 \gamma_{D}+(T-1)\left(2 \gamma_{D}-\gamma_{C L}\right)
$$

and

$$
\lim _{\mathrm{SNR} \rightarrow \infty} \frac{h(Y \mid X)}{\log (\mathrm{SNR})}=2 \gamma_{D} .
$$

Hence for $T=2$

$$
\lim _{\mathrm{SNR} \rightarrow \infty} \frac{1}{2} \frac{I(X ; Y)}{\log (\mathrm{SNR})} \geq\left(\gamma_{D}-\frac{1}{2} \gamma_{C L}\right)
$$

is achievable. Hence the outer bounds for all regimes of $T$ from Table $I$ are achievable.

\section{APPENDIX E}

\section{GAUSSIAN CODEBOOKS FOR $M \times M$ MIMO CHANNELS}

Here we prove Theorem 8 for an $M \times M$ MIMO channel (Figure 8) with coherence time $T>M$ and with exponents $\gamma_{D}$ in the direct links and $\gamma_{C L}$ in the crosslinks $\left(\gamma_{D}>\gamma_{C L}\right)$. We consider i.i.d. Gaussian codebooks across antennas and time periods and prove that a gDoF of $M\left(\left(1-\frac{1}{T}\right) \gamma_{D}-\frac{M-1}{T} \gamma_{C L}\right)$ is achievable. Using Gaussian codebooks, the rate $R \geq$ $I(G X+W ; X)$ is achievable, where

$$
\begin{gathered}
X=\left[\begin{array}{ccc}
X_{11} & \cdots & X_{1 T} \\
\vdots & & \vdots \\
X_{M 1} & \cdots & X_{M T}
\end{array}\right]=\left[\begin{array}{lll}
\overline{X_{1}} & \ldots & \overline{X_{T}}
\end{array}\right] \\
\overline{X_{i}}=\operatorname{Tran}\left[\begin{array}{lll}
X_{1 i} & \ldots & X_{M i}
\end{array}\right]
\end{gathered}
$$

with all of the elements of the $M \times T$ matrix $X$ being i.i.d. $\mathcal{C N}(0,1)$ and $W$ being an $M \times T$ matrix with i.i.d. $\mathcal{C N}(0,1)$ noise elements. The channel matrix

$$
G=\left[\begin{array}{cccc}
g_{11} & g_{12} & \cdot & g_{1 M} \\
g_{21} & g_{22} & \cdot & \cdot \\
\cdot & \cdot & \cdot & \cdot \\
g_{M 1} & \cdot & \cdot & g_{M M}
\end{array}\right]
$$

has independent elements with $g_{i i} \sim \mathcal{C N}\left(0, \mathrm{SNR}^{\gamma_{D}}\right)$ and rest of the elements distributed according to $\mathcal{C N}\left(0, \mathrm{SNR}^{\gamma_{C L}}\right)$. We will show that the mutual information satisfies

$$
I(G X+W ; X) \geq M\left((T-1) \gamma_{D}-(M-1) \gamma_{C L}\right) \log (\mathrm{SNR})
$$


We have

$$
\begin{aligned}
I(G X+W ; X)= & h(G X+W)-h(G X+W \mid X) \\
\geq h & (G X+W \mid G) \\
& -h(G X+W \mid X) .
\end{aligned}
$$

Now

$$
\begin{aligned}
& h(G X+W \mid G) \\
& \stackrel{(i)}{\geq} h(G X \mid G, W) \\
& =h(G X \mid G) \\
& \stackrel{(i i)}{=} T \times h\left(G \overline{X_{1}} \mid G\right) \\
& \stackrel{(i i i)}{=} T \mathbb{E}\left[\log \left(|\operatorname{det}(\pi e G)|^{2}\right)\right] \\
& \stackrel{(i v)}{=} T M \gamma_{D} \log (\mathrm{SNR}),
\end{aligned}
$$

where $(i)$ is using the fact that conditioning reduces entropy and conditioning on $W,(i i)$ is using the structure of $X$ from $(173)$ and the fact that elements $X_{i j}$ are i.i.d. Gaussian, (iii) is again using the fact that $X_{i j}$ are i.i.d. Gaussian and $(i v)$ is by repeated application of Lemma 9 on page 16, Tower property of expectation on Gaussian distributed $g_{i j}$ and the structure of the determinant involved. Now we will show that

$$
h(G X+W \mid X) \leq M\left(\gamma_{D} \log (\mathrm{SNR})+(M-1) \gamma_{C L} \log (\mathrm{SNR})\right)
$$

and will complete the proof.

$$
\begin{aligned}
& h(G X+W \mid X) \\
& \stackrel{(i)}{\leq} \sum_{i} h\left(\left[\begin{array}{llll}
g_{i 1} & g_{i 2} & \cdot & g_{i M}
\end{array}\right] X+\underline{W_{i}} \mid X\right) \\
& \stackrel{(i i)}{=} M h\left(\left[\begin{array}{llll}
g_{11} & g_{12} & \cdot & g_{1 M}
\end{array}\right] X+\underline{W_{1}} \mid X\right),
\end{aligned}
$$

where $(i)$ is using the fact that conditioning reduces entropy and $\underline{W_{i}}$ is a $1 \times T$ vector with i.i.d. $\mathcal{C N}(0,1)$ elements, $(i i)$ is by symmetry of the channel with $g_{i i} \sim \mathcal{C N}\left(0, \operatorname{SNR}^{\gamma_{D}}\right)$ and rest of the $g_{i j}$ distributed according to $\mathcal{C N}\left(0, \mathrm{SNR}^{\gamma_{C L}}\right)$ and the i.i.d. nature of $X_{i j}$. Now we will show that

$$
h\left(\left[\begin{array}{llll}
g_{11} & g_{12} & \cdot & g_{1 M}
\end{array}\right] X+\underline{W_{1}} \mid X\right) \dot{\leq} \gamma_{D} \log (\mathrm{SNR})+(M-1) \gamma_{C L} \log (\mathrm{SNR})
$$


and will complete the proof. Let us denote $\left[\begin{array}{llll}w_{11} & w_{12} & \cdot & w_{1 T}\end{array}\right]=\underline{W_{1}}, \underline{g_{1}}=\left[\begin{array}{llll}g_{11} & g_{12} & \cdot & g_{1 M}\end{array}\right]$. We have

$$
\begin{aligned}
h & \left(\underline{g_{1}} X+\underline{W_{1}} \mid X\right) \\
\leq & h\left(\underline{g_{1}} \overline{X_{1}}+w_{11} \mid X\right) \\
& +\sum_{i=2}^{M} h\left(\underline{g_{1}} \overline{X_{i}}+w_{1 i} \mid X, \underline{g_{1}} \overline{X_{1}}+w_{11}\right) \\
& +\sum_{i=M+1}^{T} h\left(\underline{g_{1}} \overline{X_{i}}+w_{1 i} \mid X,\left\{\underline{g_{1}} \overline{X_{k}}+w_{1 k}\right\}_{k=1}^{M}\right) .
\end{aligned}
$$

Now for the first term in $(188)$, we have

$$
\begin{aligned}
h\left(\underline{g_{1}} \overline{X_{1}}+w_{11} \mid X\right) & =h\left(\sum_{j=1}^{M} g_{j 1} X_{j 1}+w_{11} \mid X_{j 1}\right) \\
& =\mathbb{E}\left[\log \left(\pi e\left(1+\left|X_{j 1}\right|^{2}\left(\rho_{D}^{2}+(M-1) \rho_{C L}^{2}\right)\right)\right)\right] \\
& \leq \gamma_{D} \log (\mathrm{SNR})
\end{aligned}
$$

using Lemma 9 and since $\gamma_{D} \geq \gamma_{C L}$. Now consider the second term in (188), $h\left(\underline{g_{1}} \overline{X_{i}}+w_{1 i} \mid X, \underline{g_{1}} \overline{X_{1}}+w_{11}\right)$. In $\underline{g_{1}}=\left[\begin{array}{llll}g_{11} & g_{12} & \cdot & g_{1 M}\end{array}\right]$, only $g_{11}$ has SNR exponent $\gamma_{D}$ and it can be removed due to the conditioning as follows:

$$
\begin{aligned}
& h\left(\underline{g_{1}} \overline{X_{i}}+w_{1 i} \mid X, \underline{g_{1}} \overline{X_{1}}+w_{11}\right) \\
& \stackrel{(i)}{\leq} h\left[\underline{\underline{g_{1}}}\left[\begin{array}{c}
0 \\
X_{11} X_{2 i}-X_{1 i} X_{21} \\
\cdot \\
X_{11} X_{M i}-X_{1 i} X_{M 1}
\end{array}\right]+X_{11} w_{1 i}-X_{1 i} w_{11} \mid X\right)-\mathbb{E}\left[\log \left(\left|X_{11}\right|\right)\right] \\
& =\mathbb{E}\left[\log \left(\pi e\left(\rho_{C L}^{2} \sum_{j=2}^{M}\left|X_{11} X_{j i}-X_{1 i} X_{j 1}\right|^{2}+\left|X_{11}\right|^{2}+\left|X_{1 i}\right|^{2}\right)\right)\right]-\mathbb{E}\left[\log \left(\left|X_{11}\right|\right)\right] \\
& \stackrel{(i i)}{\doteq} \gamma_{C L} \log (\mathrm{SNR}),
\end{aligned}
$$

where $(i)$ is by multiplying $\underline{g_{1}} \overline{X_{i}}+w_{1 i}$ with $X_{11}$ and subtracting $X_{1 i}\left(\underline{g_{1}} \overline{X_{1}}+w_{11}\right)$ from it and using the fact that conditioning reduces entropy, and $(i i)$ is by repeated application of Lemma 9 and Tower property of expectation on Gaussian distributed $X_{i j}$.

Now consider the last term in (188)

$$
h\left(\underline{g_{1}} \overline{X_{i}}+w_{1 i} \mid X,\left\{\underline{g_{1}} \overline{X_{k}}+w_{1 k}\right\}_{k=1}^{M}\right) .
$$




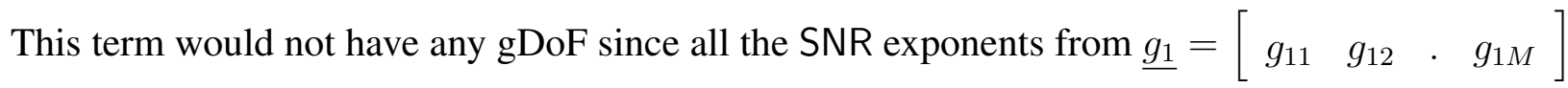
can be canceled due to availability of $M$ linear equations in the conditioning. Let

$$
X_{M \times M}=\left[\begin{array}{ccc}
X_{11} & \ldots & X_{1 M} \\
\vdots & & \vdots \\
X_{M 1} & \ldots & X_{M M}
\end{array}\right], \underline{w_{1}}=\left[\begin{array}{lll}
w_{11} & \ldots & w_{1 M}
\end{array}\right]
$$

In the conditioning, $\underline{g_{1}} X_{M \times M}+\underline{w_{1}}$ and $X_{M \times M}$ are available. Let $\operatorname{Adj}\left(X_{M \times M}\right)$ be the adjoint

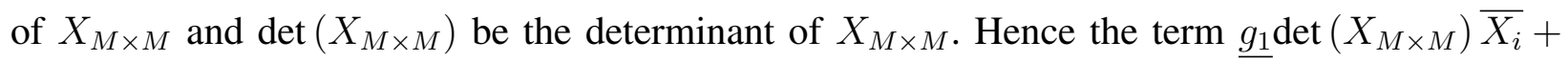
$\underline{w_{1}} \operatorname{Adj}\left(X_{M \times M}\right) \overline{X_{i}}$ is available in the conditioning. The $M$ linear equations in the conditioning can cancel off the $\mathrm{gDoF}$ contribution from $\underline{g_{1}}=\left[\begin{array}{llll}g_{11} & g_{12} & \cdot & g_{1 M}\end{array}\right]$ only if $\operatorname{det}\left(X_{M \times M}\right)$ is non-zero. Since $X$ is Gaussian i.i.d., this is true almost surely. We handle this more precisely in the following steps:

$$
\begin{aligned}
& h\left(\underline{g_{1}} \overline{X_{i}}+w_{1 i} \mid X,\left\{\underline{g_{1}} \overline{X_{k}}+w_{1 k}\right\}_{k=1}^{M}\right) \\
& \stackrel{(i)}{\leq} h\left(\underline{g_{1}} \overline{X_{i}}+w_{1 i} \mid X, \underline{g_{1}} \operatorname{det}\left(X_{M \times M}\right) \overline{X_{i}}+\underline{w_{1}} \operatorname{Adj}\left(X_{M \times M}\right) \overline{X_{i}}\right) \\
& \stackrel{(i i)}{=} h\left(\underline{g_{1}} \operatorname{det}\left(X_{M \times M}\right) \overline{X_{i}}+\operatorname{det}\left(X_{M \times M}\right) w_{1 i} \mid X, \underline{g_{1}} \operatorname{det}\left(X_{M \times M}\right) \overline{X_{i}}+\underline{w_{1}} \operatorname{Adj}\left(X_{M \times M}\right) \overline{X_{i}}\right) \\
& \quad-\mathbb{E}\left[\log \left(\left|\operatorname{det}\left(X_{M \times M}\right)\right|\right)\right] \\
& \stackrel{(i i i)}{\leq} h\left(w_{1 i} \operatorname{det}\left(X_{M \times M}\right)-\underline{w_{1}} \operatorname{Adj}\left(X_{M \times M}\right) \overline{X_{i}} \mid X\right) \\
& \quad-\mathbb{E}\left[\log \left(\operatorname{det}\left(X_{M \times M}\right)\right)\right] \\
& \stackrel{(i v)}{\leq} h\left(w_{1 i} \operatorname{det}\left(X_{M \times M}\right)-\underline{w_{1}} \operatorname{Adj}\left(X_{M \times M}\right) \overline{X_{i}} \mid X\right) \\
& \stackrel{(v)}{=} \log \left(\mathbb{E}\left[\left|\operatorname{det}\left(X_{M \times M}\right)\right|^{2}+\left\|\operatorname{Adj}\left(X_{M \times M}\right) \overline{X_{i}}\right\|^{2}\right]\right)+\log (\pi e) \\
& (v i) \\
& \quad \leq 0
\end{aligned}
$$

where $(i)$ is using the availability of $\underline{g_{1}} \operatorname{det}\left(X_{M \times M}\right) \overline{X_{i}}+\underline{w_{1}} \operatorname{Adj}\left(X_{M \times M}\right) \overline{X_{i}}$ in conditioning and using the fact that conditioning reduces entropy, $(i i)$ is by multiplying with $\operatorname{det}\left(X_{M \times M}\right)$ and compensating with $-\mathbb{E}\left[\log \left(\left|\operatorname{det}\left(X_{M \times M}\right)\right|\right)\right]$ since $\operatorname{det}\left(X_{M \times M}\right)$ is known from the values in conditioning, $(\mathrm{iii})$ is by subtracting the term available from conditioning and using the fact that conditioning reduces entropy, $(i v)$ is because $\mathbb{E}\left[\log \left(\left|\operatorname{det}\left(X_{M \times M}\right)\right|\right)\right]$ is finite by repeated 
application of Lemma 9 and the Tower property of expectation on Gaussian distributed $X_{i j},(v)$ is because $w_{1 k} \sim \mathcal{C N}(0,1)$ i.i.d. and $i>M$ and $(v i)$ is because $X_{i j} \sim \mathcal{C N}(0,1)$ i.i.d.

Now by substituting (200), (193) and (190) in (188), we get the desired result.

\section{APPENDIX F}

\section{OUTER BOUND FOR THE MISO CHANNEL WITH $T<M$}

Here we prove the gDoF outer bound given in Theorem 6 for the $M \times 1$ MISO system with $1<T<M$. The steps follow similar to the case with $T \geq M$, given in Section IV-C. We have the structure of input distribution as $X=L Q$ with

$$
L=\left[\begin{array}{cccc}
x_{11} & 0 & 0 & \\
\cdot & \cdot & 0 & 0 \\
\cdot & \cdot & \cdot & 0 \\
\cdot & \cdot & \cdot & x_{T T} \\
\cdot & \cdot & \cdot & \cdot \\
x_{M 1} & \cdot & \cdot & x_{M T}
\end{array}\right] .
$$

For the channel we have, $G=\left[g_{11} . . g_{1 M}\right], g_{1 i} \sim \mathcal{C N}\left(0, \rho_{1 i}^{2}\right), \rho_{1 i}^{2}=\operatorname{SNR}^{\gamma_{1 i}}, Y=$ $G X+W$, where $W$ is a $1 \times T$ vector with i.i.d. $\mathcal{C N}(0,1)$ components. We assume $\rho_{11}^{2} \geq \rho_{1 i}^{2}$ without loss of generality. Now note that $W Q$ has the same distribution as $W$ and is independent of $Q$ (using the fact that $W$ is isotropically distributed). Hence

$$
\begin{aligned}
& Y=(G L+W) Q \\
& =\left[\begin{array}{lll}
\left(w_{11}+\sum_{i=1}^{M} x_{i 1} g_{1 i}\right) & \left(w_{12}+\sum_{i=2}^{M} x_{i 2} g_{1 i}\right) \\
. & \cdot\left(w_{1 T}+\sum_{i=T}^{M} x_{i 2} g_{1 i}\right)
\end{array}\right] Q .
\end{aligned}
$$

Now using Lemma 13 from page 18 , we get

$$
\begin{aligned}
& h(Y)=h\left(\sum_{j=1}^{T}\left|w_{1 j}+\sum_{i=j}^{M} x_{i j} g_{1 i}\right|^{2}\right) \\
&+(T-1) \mathbb{E}\left[\log \left(\sum_{j=1}^{T}\left|w_{1 j}+\sum_{i=j}^{M} x_{i j} g_{1 i}\right|^{2}\right)\right]+\log \left(\frac{\pi^{T}}{\Gamma(T)}\right) \\
& \stackrel{(i)}{\leq} h\left(\sum_{j=1}^{T}\left|w_{1 j}+\sum_{i=j}^{M} x_{i j} g_{1 i}\right|^{2}\right) \\
&+(T-1) \mathbb{E}\left[\log \left(\sum_{j=1}^{T}\left(1+\sum_{i=j}^{M}\left|x_{i j}\right|^{2} \rho_{i j}^{2}\right)\right)\right]+\log \left(\frac{\pi^{T}}{\Gamma(T)}\right)
\end{aligned}
$$




$$
\begin{aligned}
& \stackrel{\text { (ii) }}{\leq} h\left(\sum_{j=1}^{T}\left|w_{1 j}+\sum_{i=j}^{M} x_{i j} g_{1 i}\right|^{2}\right) \\
& +(T-1) \mathbb{E}\left[\log \left(\sum_{i=1}^{M} \rho_{1 i}^{2}\left(\sum_{j=1}^{\min (i, T)}\left|x_{i j}\right|^{2}\right)+T\right)\right]+\log \left(\frac{\pi^{T}}{\Gamma(T)}\right),
\end{aligned}
$$

where $(i)$ is using the Tower property of expectation and Jensen's inequality and (ii) is using $\sum_{j=1}^{T} \sum_{i=j}^{M}\left|x_{i j}\right|^{2} \rho_{1 i}^{2}=\sum_{i=1}^{M} \sum_{j=1}^{\min (i, T)}\left|x_{i j}\right|^{2} \rho_{1 i}^{2}$. Now using 28 , we have

$$
\begin{aligned}
h(Y \mid X)=\mathbb{E}[ & {\left[\log \left(\operatorname{det}\left(L^{\dagger} \operatorname{diag}\left(\rho_{11}^{2}, \ldots, \rho_{1 M}^{2}\right) L+I_{T}\right)\right)\right] } \\
& +(T) \log (\pi e) \\
=\mathbb{E} & {\left[\log \left(\prod_{i=1}^{M}\left(1+\omega_{i}\right)\right)\right]+T \log (\pi e), }
\end{aligned}
$$

where $\omega_{i}$ are the eigenvalues of $L^{\dagger} \operatorname{diag}\left(\rho_{11}^{2}, \ldots, \rho_{1 M}^{2}\right) L$. Hence

$$
\begin{aligned}
h(Y \mid X) & =\mathbb{E}\left[\log \left(\prod_{i=1}^{M}\left(1+\omega_{i}\right)\right)\right]+T \log (\pi e) \\
& \geq \mathbb{E}\left[\log \left(1+\sum \omega_{i}\right)\right]+T \log (\pi e) .
\end{aligned}
$$

The last step is true because $\omega_{i} \geq 0$. Now

$$
\begin{aligned}
\sum \omega_{i} & =\operatorname{Trace}\left(L^{\dagger} \operatorname{diag}\left(\rho_{11}^{2}, \ldots, \rho_{1 M}^{2}\right) L\right) \\
& =\operatorname{Trace}\left(\operatorname{diag}\left(\rho_{11}^{2}, \ldots, \rho_{1 M}^{2}\right) L L^{\dagger}\right) \\
& =\sum_{i=1}^{M} \rho_{1 i}^{2}\left(\sum_{j=1}^{\min (i, T)}\left|x_{i j}\right|^{2}\right)
\end{aligned}
$$

Hence

$$
h(Y \mid X) \geq \mathbb{E}\left[\log \left(1+\sum_{i=1}^{M} \rho_{1 i}^{2}\left(\sum_{j=1}^{\min (i, T)}\left|x_{i j}\right|^{2}\right)\right)\right]+T \log (\pi e)
$$

Hence

$$
\begin{aligned}
I(X ; Y) \leq & h\left(\sum_{j=1}^{M}\left|w_{1 j}+\sum_{i=j}^{M} x_{i j} g_{1 i}\right|^{2}+\sum_{i=M+1}^{T}\left|w_{1 i}\right|^{2}\right) \\
& +(T-2) \mathbb{E}\left[\log \left(\sum_{i=1}^{M} \rho_{1 i}^{2}\left(\sum_{j=1}^{i}\left|x_{i j}\right|^{2}\right)+T\right)\right] \\
& +\log \left(\frac{\pi^{T}}{\Gamma(T)}\right)-T \log (\pi e)
\end{aligned}
$$




$$
\dot{\leq}(T-1) \log \left(\sum_{i=1}^{M} \rho_{1 i}^{2} M T+T\right),
$$

where the last step was using Lemma 15 and Jensen's inequality. Hence

$$
\limsup _{\text {SNR } \rightarrow \infty} \frac{I(X ; Y)}{\log (\mathrm{SNR})} \leq(T-1) \gamma_{11} \text {. }
$$

\section{APPENDIX G}

\section{PROOF OF LEMMA 19}

Here we prove that $h\left(\left|a g_{11}+b g_{12}+w_{11}\right|^{2}+\left|c g_{12}+w_{12}\right|^{2}+\sum_{i=3}^{T}\left|w_{1 i}\right|^{2}\right)$ and

$$
\mathbb{E}\left[\log \left(|a|^{2} \rho_{11}^{2}+\left(|b|^{2}+|c|^{2}\right) \rho_{12}^{2}+1\right)\right]
$$

have the same gDoF. For this, consider the point to point channel

$$
\mathcal{C}_{1}: V=\left|a g_{11}+b g_{12}+w_{11}\right|^{2}+\left|c g_{12}+w_{12}\right|^{2}+\sum_{i=3}^{T}\left|w_{1 i}\right|^{2}
$$

with inputs $a, b, c$ and power constraint $T$. Its capacity is given by

$$
\begin{aligned}
C_{1}=\max _{p(a, b, c) ; \mathbb{E}\left[|a|^{2}+|b|^{2}+|c|^{2}\right] \leq T}\left\{h\left(\left(\left|a g_{11}+b g_{12}+w_{11}\right|^{2}+\left|c g_{12}+w_{12}\right|^{2}+\sum_{i=3}^{T}\left|w_{1 i}\right|^{2}\right)\right)\right. \\
\left.-h\left(\left|a g_{11}+b g_{12}+w_{11}\right|^{2}+\left|c g_{12}+w_{12}\right|^{2}+\sum_{i=3}^{T}\left|w_{1 i}\right|^{2} \mid a, b, c\right)\right\} .
\end{aligned}
$$

From [7, (32)] we have

$$
\begin{aligned}
I(U ; V) \leq \mathbb{E}[ & {[\log (V)]-h(V \mid U)+\log (\Gamma(\alpha)) } \\
& +\alpha(1+\log (\mathbb{E}[V])-\mathbb{E}[\log (V)])-\alpha \log (\alpha)
\end{aligned}
$$

for any $\alpha>0$ for channels whose output $V$ takes values in $\mathbb{R}^{+}$. We will use this result to bound $I(U ; V)$ for any input distribution $p(a, b, c) ; \mathbb{E}\left[|a|^{2}+|b|^{2}+|c|^{2}\right] \leq T$ for the channel $\mathcal{C}_{1}$ with $U=(a, b, c)$ as input. Now

$$
\begin{aligned}
& h(V \mid U) \\
& =h\left(\left|a g_{11}+b g_{12}+w_{11}\right|^{2}+\left|c g_{12}+w_{12}\right|^{2}+\sum_{i=3}^{T}\left|w_{1 i}\right|^{2} \mid a, b, c\right) \\
& \stackrel{(i)}{\leq} \mathbb{E}\left[\log \left(e \mathbb{E}\left[\left|a g_{11}+b g_{12}+w_{11}\right|^{2}+\left|c g_{12}+w_{12}\right|^{2}+\sum_{i=3}^{T}\left|w_{1 i}\right|^{2} \mid a, b, c\right]\right)\right]
\end{aligned}
$$




$$
\begin{aligned}
& \stackrel{(i i)}{=} \mathbb{E}\left[\log \left(e\left(\left(\rho_{11}^{2}|a|^{2}+\rho_{12}^{2}|b|^{2}+1\right)+\left(\rho_{12}^{2}|c|^{2}+1\right)+(T-2)\right)\right)\right] \\
& =\mathbb{E}\left[\log \left(\rho_{11}^{2}|a|^{2}+\rho_{12}^{2}|b|^{2}+\rho_{12}^{2}|c|^{2}+T\right)\right]+\log (e)
\end{aligned}
$$

where $(i)$ was using the definition of conditional entropy and Lemma 15, (ii) was using the fact that given $(a, b, c), a g_{11}+b g_{12}+w_{11}, c g_{12}+w_{12}$ are sums of independent Gaussians. Note that

$$
\begin{aligned}
\mathbb{E}[\log (V)] & =\mathbb{E}\left[\mathbb{E}\left[\log \left(\left|a g_{11}+b g_{12}+w_{11}\right|^{2}+\left|c g_{12}+w_{12}\right|^{2}+\sum_{i=3}^{T}\left|w_{1 i}\right|^{2} \mid a, b, c\right)\right]\right] \\
& \leq \mathbb{E}\left[\log \left(\rho_{11}^{2}|a|^{2}+\rho_{12}^{2}|b|^{2}+\rho_{12}^{2}|c|^{2}+T\right)\right]
\end{aligned}
$$

using Jensen's inequality. Also

$$
\begin{aligned}
\mathbb{E}[\log (V)] & =\mathbb{E}\left[\mathbb{E}\left[\log \left(\left|a g_{11}+b g_{12}+w_{11}\right|^{2}+\left|c g_{12}+w_{12}\right|^{2}+\sum_{i=3}^{T}\left|w_{1 i}\right|^{2} \mid a, b, c\right)\right]\right] \\
& \geq \mathbb{E}\left[\log \left(\rho_{11}^{2}|a|^{2}+\rho_{12}^{2}|b|^{2}+\rho_{12}^{2}|c|^{2}+T\right)\right]-3 \gamma_{E} \log (e)
\end{aligned}
$$

by using Lemma 9 on page 16 for exponentially distributed $\left|a g_{r d 2}+b g_{r d 1}+w_{d 1}\right|^{2},\left|c g_{r d 1}+w_{d 2}\right|^{2}$ (for given $a, b, c$ ) and Lemma 10 for chi-squared distributed $\sum_{i=3}^{T}\left|w_{d i}\right|^{2}$.

Lemma 24. The term $\mathbb{E}[\log (V)]-h(V \mid U)$ is upper bounded by $\log (3+T)+\frac{7}{2} \log (e)$ independent of SNR.

Proof: It suffices to show that for any constant $\left(a^{\prime}, b^{\prime}, c^{\prime}\right), \mathbb{E}\left[\log (V) \mid U=\left(a^{\prime}, b^{\prime}, c^{\prime}\right)\right]-$ $h\left(V \mid U=\left(a^{\prime}, b^{\prime}, c^{\prime}\right)\right) \leq \log (3+T)+\frac{7}{2} \log (e)$ independent of $a^{\prime}, b^{\prime}, c^{\prime}$ and SNR.

$$
\begin{aligned}
\mathbb{E}\left[\log (V) \mid U=\left(a^{\prime}, b^{\prime}, c^{\prime}\right)\right]-h\left(V \mid U=\left(a^{\prime}, b^{\prime}, c^{\prime}\right)\right) \\
=\mathbb{E}\left[\log \left(\left|a^{\prime} g_{11}+b^{\prime} g_{12}+w_{11}\right|^{2}+\left|c^{\prime} g_{12}+w_{12}\right|^{2}+\sum_{i=3}^{T}\left|w_{1 i}\right|^{2}\right)\right] \\
-h\left(\left|a^{\prime} g_{11}+b^{\prime} g_{12}+w_{11}\right|^{2}+\left|c^{\prime} g_{12}+w_{12}\right|^{2}+\sum_{i=3}^{T}\left|w_{1 i}\right|^{2}\right) \\
\stackrel{(i)}{\leq} \log \left(\mathbb{E}\left[\left|a^{\prime} g_{11}+b^{\prime} g_{12}+w_{11}\right|^{2}+\left|c^{\prime} g_{12}+w_{12}\right|^{2}+\sum_{i=3}^{T}\left|w_{1 i}\right|^{2}\right]\right) \\
\quad-h\left(\left|a^{\prime} g_{11}+b^{\prime} g_{12}+w_{11}\right|^{2}+\left|c^{\prime} g_{12}+w_{12}\right|^{2}\right) \\
\stackrel{(i i)}{=} \log \left(\rho_{11}^{2}\left|a^{\prime}\right|^{2}+\rho_{12}^{2}\left|b^{\prime}\right|^{2}+\rho_{12}^{2}\left|c^{\prime}\right|^{2}+T\right)
\end{aligned}
$$




$$
-h\left(\left|a^{\prime} \rho_{11} \eta_{11}+b^{\prime} \rho_{12} \eta_{12}+w_{11}\right|^{2}+\left|c^{\prime} \rho_{12} \eta_{12}+w_{12}\right|^{2}\right),
$$

where $(i)$ is using Jensen's inequality and the fact that conditioning reduces entropy to remove $\sum_{i=3}^{T}\left|w_{d i}\right|^{2}$ in the negative term; $(i i)$ is using the fact that $a^{\prime} g_{11}+b^{\prime} g_{12}+w_{11}, c^{\prime} g_{12}+w_{12}$ are sums of independent Gaussians. We also introduced $\eta_{i j} \sim \mathcal{C N}(0,1)$ in $(i i)$ so that $g_{i j}=\rho_{i j} \eta_{i j}$.

Consider the case when $1 \leq \max \left(\left|a^{\prime} \rho_{11}\right|,\left|b^{\prime} \rho_{12}\right|,\left|c^{\prime} \rho_{12}\right|\right)$. Assume $1 \leq\left|b^{\prime} \rho_{12}\right|=$ $\max \left(\left|a^{\prime} \rho_{11}\right|,\left|b^{\prime} \rho_{12}\right|,\left|c^{\prime} \rho_{12}\right|\right)$

$$
\begin{aligned}
\mathbb{E}[\log (V) \mid U & \left.=\left(a^{\prime}, b^{\prime}, c^{\prime}\right)\right]-h\left(V \mid U=\left(a^{\prime}, b^{\prime}, c^{\prime}\right)\right) \\
\leq & \log \left(\rho_{11}^{2}\left|a^{\prime}\right|^{2}+\rho_{12}^{2}\left|b^{\prime}\right|^{2}+\rho_{12}^{2}\left|c^{\prime}\right|^{2}+T\right)-\log \left(\rho_{12}^{2}\left|b^{\prime}\right|^{2}\right) \\
& -h\left(\left|\frac{a^{\prime} \rho_{11}}{b^{\prime} \rho_{12}} \eta_{11}+\eta_{12}+\frac{w_{11}}{b^{\prime} \rho_{12}}\right|^{2}+\left|\frac{c^{\prime}}{b^{\prime}} \eta_{12}+\frac{w_{12}}{b^{\prime} \rho_{12}}\right|^{2}\right) .
\end{aligned}
$$

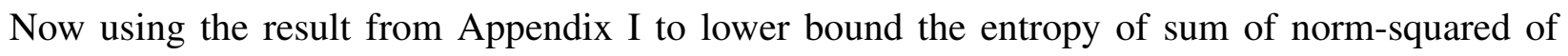
Gaussian vectors, we have

$$
\begin{aligned}
& h\left(\left|\frac{a^{\prime} \rho_{11}}{b^{\prime} \rho_{12}} \eta_{11}+\eta_{12}+\frac{w_{11}}{b^{\prime} \rho_{12}}\right|^{2}+\left|\frac{c^{\prime}}{b^{\prime}} \eta_{12}+\frac{w_{12}}{b^{\prime} \rho_{12}}\right|^{2}\right) \\
& \geq h\left(\left|\frac{a^{\prime} \rho_{11}}{b^{\prime} \rho_{12}} \eta_{11}+\eta_{12}+\frac{w_{11}}{b^{\prime} \rho_{12}}\right|^{2}+\left|\frac{c^{\prime}}{b^{\prime}} \eta_{12}+\frac{w_{12}}{b^{\prime} \rho_{12}}\right|^{2} \mid \eta_{11}\right) \\
& \geq-\frac{7}{2} \log (e) .
\end{aligned}
$$

Hence we get

$$
\begin{aligned}
& \mathbb{E}\left[\log (V) \mid U=\left(a^{\prime}, b^{\prime}, c^{\prime}\right)\right]-h\left(V \mid U=\left(a^{\prime}, b^{\prime}, c^{\prime}\right)\right) \\
& \quad \leq \log \left(\rho_{11}^{2}\left|a^{\prime}\right|^{2}+\rho_{12}^{2}\left|b^{\prime}\right|^{2}+\rho_{12}^{2}\left|c^{\prime}\right|^{2}+T\right)-\log \left(\rho_{12}^{2}\left|b^{\prime}\right|^{2}\right)+\frac{7}{2} \log (e) \\
& \quad \stackrel{(i)}{\leq} \log (3+T)+\frac{7}{2} \log (e),
\end{aligned}
$$

where in step $(i)$ we used $1 \leq\left|b^{\prime} \rho_{12}\right| \leq \max \left(\left|a^{\prime} \rho_{11}\right|,\left|b^{\prime} \rho_{12}\right|,\left|c^{\prime} \rho_{12}\right|\right)$.

Similarly for other cases $1 \leq\left|a^{\prime} \rho_{11}\right|=\max \left(\left|a^{\prime} \rho_{11}\right|,\left|b^{\prime} \rho_{12}\right|,\left|c^{\prime} \rho_{12}\right|\right)$ and $1 \leq$ $\left|c^{\prime} \rho_{12}\right|=\max \left(\left|a^{\prime} \rho_{11}\right|,\left|b^{\prime} \rho_{12}\right|,\left|c^{\prime} \rho_{12}\right|\right)$, we can show that $\mathbb{E}\left[\log (V) \mid U=\left(a^{\prime}, b^{\prime}, c^{\prime}\right)\right]-$ $h\left(V \mid U=\left(a^{\prime}, b^{\prime}, c^{\prime}\right)\right)$ is upper bounded by $\log (3+T)+\frac{7}{2} \log (e)$.

Now if $1>\max \left(\left|a^{\prime} \rho_{11}\right|,\left|b^{\prime} \rho_{12}\right|,\left|c^{\prime} \rho_{12}\right|\right)$

$\mathbb{E}\left[\log (V) \mid U=\left(a^{\prime}, b^{\prime}, c^{\prime}\right)\right]-h\left(V \mid U=\left(a^{\prime}, b^{\prime}, c^{\prime}\right)\right)$ 


$$
\begin{aligned}
& \leq \log \left(\rho_{11}^{2}\left|a^{\prime}\right|^{2}+\rho_{12}^{2}\left|b^{\prime}\right|^{2}+\rho_{12}^{2}\left|c^{\prime}\right|^{2}+T\right) \\
& \quad-h\left(\left|a^{\prime} \rho_{11} \eta_{11}+b^{\prime} \rho_{12} \eta_{12}+w_{11}\right|^{2}+\left|c^{\prime} \rho_{12} \eta_{12}+w_{12}\right|^{2}\right) \\
& \stackrel{(i)}{\leq} \log (3+T)-h\left(\left|a^{\prime} \rho_{11} \eta_{11}+b^{\prime} \rho_{12} \eta_{12}+w_{11}\right|^{2}+\left|c^{\prime} \rho_{12} \eta_{12}+w_{12}\right|^{2} \mid \eta_{11}, \eta_{12}, w_{12}\right) \\
& =\log (3+T)-h\left(\left|a^{\prime} \rho_{11} \eta_{11}+b^{\prime} \rho_{12} \eta_{12}+w_{11}\right|^{2} \mid \eta_{11}, \eta_{12}\right) \\
& \stackrel{(i i)}{\leq} \log (3+T)+\frac{7}{2} \log (e)
\end{aligned}
$$

where in $\operatorname{step}(i)$ we used the fact $1>\max \left(\left|a^{\prime} \rho_{11}\right|,\left|b^{\prime} \rho_{12}\right|,\left|c^{\prime} \rho_{12}\right|\right)$ and the fact that conditioning reduces entropy, in step $(i i)$ we used the result from Appendix II to lower bound $h\left(\left|a^{\prime} \rho_{11} \eta_{11}+b^{\prime} \rho_{12} \eta_{12}+w_{11}\right|^{2} \mid \eta_{11}, \eta_{12}\right)$.

Using (222), (223), 224) and using Lemma 24, we get

$$
\begin{aligned}
\mathbb{E}[\log (V)] & \doteq \mathbb{E}\left[\log \left(\rho_{11}^{2}|a|^{2}+\rho_{12}^{2}|b|^{2}+\rho_{12}^{2}|c|^{2}+T\right)\right] \\
& \doteq h\left(\left|a g_{11}+b g_{12}+w_{11}\right|^{2}+\left|c g_{12}+w_{12}\right|^{2}+\sum_{i=3}^{T}\left|w_{1 i}\right|^{2} \mid a, b, c\right)=h(V \mid U)
\end{aligned}
$$

and the above approximation is tight within a constant independent of SNR. Hence it follows that

$$
\begin{array}{r}
C_{1} \doteq \max _{p(a, b, c) ; \mathbb{E}\left[|a|^{2}+|b|^{2}+|c|^{2}\right] \leq T}\left\{h\left(\left|a g_{11}+b g_{12}+w_{11}\right|^{2}+\left|c g_{12}+w_{12}\right|^{2}+\sum_{i=3}^{T}\left|w_{1 i}\right|^{2}\right)\right. \\
\left.-\mathbb{E}\left[\log \left(\rho_{11}^{2}|a|^{2}+\rho_{12}^{2}|b|^{2}+\rho_{12}^{2}|c|^{2}+T\right)\right]\right\}
\end{array}
$$

and the above equality is tight within a constant independent of SNR. Now we shall prove that

$$
\limsup _{\text {SNR } \rightarrow \infty} C_{1}(\mathrm{SNR})-\log (\log (\mathrm{SNR}))<\infty
$$

and hence it will prove our claim that $h\left(\left|a g_{11}+b g_{12}+w_{11}\right|^{2}+\left|c g_{12}+w_{12}\right|^{2}+\sum_{i=3}^{T}\left|w_{1 i}\right|^{2}\right)$ and $\mathbb{E}\left[\log \left(\rho_{11}^{2}|a|^{2}+\rho_{12}^{2}|b|^{2}+\rho_{12}^{2}|c|^{2}+T\right)\right]$ have the same gDoF.

Now looking at 218) again, if the term $\log (\mathbb{E}[V])-\mathbb{E}[\log (V)]$ does not approach infinity with the SNR, then the result follows directly by choosing any fixed $\alpha>0$. When $\log (\mathbb{E}[V])-$ $\mathbb{E}[\log (V)]$ does tend to infinity with SNR, we choose

$$
\alpha^{*}=(1+\log (\mathbb{E}[V])-\mathbb{E}[\log (V)])^{-1}
$$


with $\alpha^{*} \downarrow 0$ with the SNR and we have $\log \left(\Gamma\left(\alpha^{*}\right)\right)=\log \left(\frac{1}{\alpha^{*}}\right)+o(1)$ and $\alpha^{*} \log \left(\alpha^{*}\right)=o(1)$ where $o(1)$ tends to zero as $\alpha^{*}$ tends to zero, following [7, (337)]. Hence using [239) and Lemma 24 on (218), we get

$$
\begin{aligned}
C_{1} & \leq \log (3+T)+\frac{7}{2} \log (e)+1+\log \left(\frac{1}{\alpha^{*}}\right)+o(1) \\
& =r_{4}+\log \left(\frac{1}{\alpha^{*}}\right)+o(1) .
\end{aligned}
$$

We let $r_{4}=\log (3+T)+(7 / 2) \log (e)+1$ in the last step. Now

$$
\begin{aligned}
& \frac{1}{\alpha^{*}}=1+\log (\mathbb{E}[V])-\mathbb{E}[\log (V)] \\
& =1+\log \left(\mathbb{E}\left[\left|a g_{11}+b g_{12}+w_{11}\right|^{2}+\left|c g_{12}+w_{12}\right|^{2}+\sum_{i=3}^{T}\left|w_{1 i}\right|^{2}\right]\right) \\
& -\mathbb{E}\left[\log \left(\left|a g_{11}+b g_{12}+w_{11}\right|^{2}+\left|c g_{12}+w_{12}\right|^{2}+\sum_{i=3}^{T}\left|w_{1 i}\right|^{2}\right)\right] \\
& \stackrel{(i)}{=} 1+\log \left(\mathbb{E}\left[\rho_{11}^{2}|a|^{2}+\rho_{12}^{2}|b|^{2}+\rho_{12}^{2}|c|^{2}+T\right]\right) \\
& -\mathbb{E}\left[\log \left(\left|a g_{11}+b g_{12}+w_{11}\right|^{2}+\left|c g_{12}+w_{12}\right|^{2}+\sum_{i=3}^{T}\left|w_{1 i}\right|^{2}\right)\right] \\
& \stackrel{(i i)}{\leq} 1+\log \left(\rho_{11}^{2} T+\rho_{12}^{2} T+T\right) \\
& -\mathbb{E}\left[\log \left(\left|a g_{11}+b g_{12}+w_{11}\right|^{2}+\left|c g_{12}+w_{12}\right|^{2}+\sum_{i=3}^{T}\left|w_{1 i}\right|^{2}\right)\right] \\
& \stackrel{(\text { iii })}{\leq} 1+\log \left(\rho_{11}^{2}+\rho_{12}^{2}+1\right)+\log (T)-\mathbb{E}\left[\mathbb{E}\left[\log \left(\left|a g_{11}+b g_{12}+w_{11}\right|^{2}+0\right) \mid a, b\right]\right] \\
& \stackrel{(i v)}{\leq} 1+\log \left(\rho_{11}^{2}+\rho_{12}^{2}+1\right)+\log (T)-\mathbb{E}\left[\log \left(\rho_{11}^{2}|a|^{2}+\rho_{12}^{2}|b|^{2}+1\right)\right]+\gamma_{E} \log (e) \\
& \stackrel{(v)}{\leq} 1+\log \left(\rho_{11}^{2}+\rho_{12}^{2}+1\right)+\log (T)-0+\gamma_{E} \log (e) \text {, }
\end{aligned}
$$

where $(i)$ is using the Tower property of expectation and that given $(a, b, c), a g_{11}+b g_{12}+$ $w_{11}, c g_{12}+w_{12}$ are sums of independent Gaussians, $(i i)$ is using power constraints on $a, b, c$, (iii) is because $\left|c g_{12}+w_{12}\right|^{2}+\sum_{i=3}^{T}\left|w_{1 i}\right|^{2}>0,(i v)$ is using Lemma 9 on page 16 and $(v)$ is because $\log \left(\rho_{11}^{2}|a|^{2}+\rho_{12}^{2}|b|^{2}+1\right)>0$. Hence

$$
C_{1} \leq r_{4}+\log \left(1+\log \left(\rho_{11}^{2}+\rho_{12}^{2}+\rho_{12}^{2}+1\right)+\log (T)+\gamma \log (e)\right)+o(1)
$$

and the proof is complete. 


\section{APPENDIX H}

\section{Proof of LEMMA 20}

Here we prove that $h\left(\left.\left|\xi_{22}\right|^{2}|| \xi_{11}\right|^{2}\right) \doteq h\left(\left.\left|\xi_{22}\right|^{2}|| \xi_{11}\right|^{2}, a, b, c\right)$ with $\left|\xi_{11}\right|^{2},\left|\xi_{22}\right|^{2}$ defined in [47, 49p on page 23 . The inequality $h\left(\left.\left|\xi_{22}\right|^{2}|| \xi_{11}\right|^{2}, a, b, c\right) \leq \mathbb{E}\left[\log \left(e \mathbb{E}\left[\left|\xi_{22}\right|^{2} \mid a, b, c\right]\right)\right]$ follows using Lemma 15 . We now only need to show that $I\left(\left|\xi_{22}\right|^{2} ; a, b,\left.c|| \xi_{11}\right|^{2}\right)$ has zero gDoF. Now

$$
I\left(\left|\xi_{22}\right|^{2} ; a, b,\left.c|| \xi_{11}\right|^{2}\right) \leq I\left(\left|\xi_{22}\right|^{2} ; a, b, c,\left|\xi_{11}\right|^{2}\right)
$$

We will show that $I\left(\left|\xi_{22}\right|^{2} ; a, b, c,\left|\xi_{11}\right|^{2}\right)$ has no gDoF. From [7, (32)] we have

$$
\begin{aligned}
I(U ; V) \leq \mathbb{E}[ & {[\log (V)]-h(V \mid U)+\log (\Gamma(\alpha)) } \\
& +\alpha(1+\log (\mathbb{E}[V])-\mathbb{E}[\log (V)])-\alpha \log (\alpha)
\end{aligned}
$$

for any $\alpha>0$ for channels whose output $V$ takes values in $\mathbb{R}^{+}$. We will use this result to bound $I\left(\left|\xi_{22}\right|^{2} ; a, b, c,\left|\xi_{11}\right|^{2}\right)$ with $U=\left(a, b, c,\left|\xi_{11}\right|^{2}\right), V=\left|\xi_{22}\right|^{2}$ for any distribution of $a, b, c$ with the power constraint $\mathbb{E}\left[|a|^{2}+|b|^{2}+|c|^{2}\right] \leq T$. The result from [7] can be applied assuming the channel induced by $p\left(\left.\left|\xi_{22}\right|^{2}|a, b, c,| \xi_{11}\right|^{2}\right)$ satisfies the Borel measurability conditions in [7. Theorem 5.1], i.e., for any given Borel set $\mathcal{B} \subset \mathbb{R}^{+}, f_{\mathcal{B}}(v)=p\left(\mathcal{B} \mid v=\left(a, b, c,\left|\xi_{11}\right|^{2}\right)\right)$ is a Borel measurable function.

Recall that from 47) and 49, we have

$$
\begin{aligned}
\left|\xi_{11}\right|^{2}= & \left|a g_{11}+b g_{12}+w_{11}\right|^{2}+\left|c g_{12}+w_{12}\right|^{2}+\sum_{i=3}^{T}\left|w_{1 i}\right|^{2} \\
\left|\xi_{22}\right|^{2}= & \left|a g_{21}+b g_{22}+w_{21}\right|^{2}+\left|c g_{22}+w_{22}\right|^{2}+\sum_{i=3}^{T}\left|w_{2 i}\right|^{2} \\
& -\frac{\left|\left(a g_{21}+b g_{22}+w_{21}\right)\left(a g_{11}+b g_{12}+w_{11}\right)^{*}+\left(c g_{22}+w_{22}\right)\left(c g_{12}+w_{12}\right)^{*}+\sum_{i=3}^{T} w_{2 i} w_{1 i}^{*}\right|^{2}}{\left|a g_{11}+b g_{12}+w_{11}\right|^{2}+\left|c g_{12}+w_{12}\right|^{2}+\sum_{i=3}^{T}\left|w_{1 i}\right|^{2}} .
\end{aligned}
$$

We first consider $\log (\mathbb{E}[V])-\mathbb{E}[\log (V)]=\mathbb{E}\left[\log \left(\left|\xi_{22}\right|^{2}\right)\right]-h\left(\left.\left|\xi_{22}\right|^{2}|a, b, c,| \xi_{11}\right|^{2}\right)$ and show that it is bounded independent of SNR. Note that we can manipulate $\left|\xi_{22}\right|^{2}$ as

$$
\begin{aligned}
& \left|\xi_{22}\right|^{2} \\
& =\left(\left|a g_{21}+b g_{22}+w_{21}\right|^{2}+\left|c g_{22}+w_{22}\right|^{2}+\sum_{i=3}^{T}\left|w_{2 i}\right|^{2}\right)
\end{aligned}
$$




$$
\begin{aligned}
& \quad-\left|\left(a g_{21}+b g_{22}+w_{21}\right) u_{1}^{*}+\left(c g_{22}+w_{22}\right) u_{2}^{*}+\sum_{i=3}^{T} w_{2 i} u_{i}^{*}\right|^{2} \\
& =\|\left[a g_{21}+b g_{22}+w_{21}, c g_{22}+w_{22}, w_{23}, \ldots, w_{2 T}\right] \\
& \quad-\left[a g_{21}+b g_{22}+w_{21}, c g_{22}+w_{22}, w_{23}, \ldots, w_{2 T}\right]\left[\begin{array}{c}
u_{1}^{*} \\
u_{2}^{*} \\
\cdot \\
\cdot \\
u_{T}^{*}
\end{array}\right]\left[u_{1}, \ldots, u_{T}\right] \|^{2},
\end{aligned}
$$

where $\|\cdot\|$ indicates 2 -norm for a vector and $\left(u_{i}\right)$ forms a unit norm complex vector

$$
\begin{gathered}
{\left[u_{1}, \ldots, u_{T}\right]=\frac{\left[a g_{11}+b g_{12}+w_{11}, c g_{12}+w_{12}, w_{13}, \ldots, w_{1 T}\right]}{\left|a g_{11}+b g_{12}+w_{11}\right|^{2}+\left|c g_{12}+w_{12}\right|^{2}+\sum_{i=3}^{T}\left|w_{1 i}\right|^{2}},} \\
\left|\xi_{22}\right|^{2}=\left|a g_{21}+b g_{22}+w_{21}-\left(\left(a g_{21}+b g_{22}+w_{21}\right) u_{1}^{*}+\left(c g_{22}+w_{22}\right) u_{2}^{*}+\sum_{i=3}^{T} w_{2 i} u_{i}^{*}\right) u_{1}\right|^{2} \\
+\left|c g_{22}+w_{22}-\left(\left(a g_{21}+b g_{22}+w_{21}\right) u_{1}^{*}+\left(c g_{22}+w_{22}\right) u_{2}^{*}+\sum_{i=3}^{T} w_{2 i} u_{i}^{*}\right) u_{2}\right| \\
+\left|w_{23}-\left(\left(a g_{21}+b g_{22}+w_{21}\right) u_{1}^{*}+\left(c g_{22}+w_{22}\right) u_{2}^{*}+\sum_{i=3}^{T} w_{2 i} u_{i}^{*}\right) u_{3}\right|^{2} \\
+\ldots \\
+\left|w_{2 T}-\left(\left(a g_{21}+b g_{22}+w_{21}\right) u_{1}^{*}+\left(c g_{22}+w_{22}\right) u_{2}^{*}+\sum_{i=3}^{T} w_{2 i} u_{i}^{*}\right) u_{T}\right|^{2} \\
\stackrel{(i)}{=} \sum_{i=1}^{T}\left|\eta_{21} \kappa_{1 i}+\eta_{22} \kappa_{2 i}+\sum_{j=1}^{T} w_{2 j} \kappa_{(j+2) i}\right|^{2}
\end{gathered}
$$

where in step $(i) \eta_{i j}$ are independent $\mathcal{C N}(0,1)$ after the substitution $g_{i j}=\rho_{i j} \eta_{i j}$, also $\kappa_{i j}$ are functions of $a, b, c, \rho_{i j}, u_{i}$ obtained after collecting the coefficients of $\eta_{i j}, w_{2 j}$. Note that $\max _{i, j}\left(\left|\kappa_{i j}\right|\right) \geq 1$. Now

$$
\mathbb{E}\left[\log \left(\left|\xi_{22}\right|^{2}\right)\right]-h\left(\left.\left|\xi_{22}\right|^{2}|a, b, c,| \xi_{11}\right|^{2}\right)
$$




$$
\begin{aligned}
=\mathbb{E} & {\left[\log \left(\sum_{i=1}^{T}\left|\eta_{21} \kappa_{1 i}+\eta_{22} \kappa_{2 i}+\sum_{j=1}^{T} w_{2 j} \kappa_{(j+2) i}\right|^{2}\right)\right] } \\
& -h\left(\left.\sum_{i=1}^{T}\left|\eta_{21} \kappa_{1 i}+\eta_{22} \kappa_{2 i}+\sum_{j=1}^{T} w_{2 j} \kappa_{(j+2) i}\right|^{2}|a, b, c,| \xi_{11}\right|^{2}\right) \\
\leq \mathbb{E} & {\left[\log \left(\sum_{i=1}^{T}\left|\eta_{21} \kappa_{1 i}+\eta_{22} \kappa_{2 i}+\sum_{j=1}^{T} w_{2 j} \kappa_{(j+2) i}\right|^{2}\right)\right] } \\
& -h\left(\sum_{i=1}^{T}\left|\eta_{21} \kappa_{1 i}+\eta_{22} \kappa_{2 i}+\sum_{j=1}^{T} w_{2 j} \kappa_{(j+2) i}\right|^{2} \mid\left\{\kappa_{i j}\right\}\right),
\end{aligned}
$$

where the last step uses the fact that conditioning reduces entropy and Markovity $\left(a, b, c,\left|\xi_{11}\right|^{2}\right)$ $-\left(\left\{\kappa_{i j}\right\}\right)-\left(\sum_{i=1}^{T}\left|\eta_{21} \kappa_{1 i}+\eta_{22} \kappa_{2 i}+\sum_{j=1}^{T} w_{2 j} \kappa_{(j+2) i}\right|^{2}\right)$. Note that $\eta_{21}, \eta_{22}, w_{2 j}$ are independent of $\kappa_{i j}$. Now it suffices to show that for any given set of constant $\kappa_{i j}^{\prime}$ the difference

$\mathbb{E}\left[\log \left(\sum_{i=1}^{T}\left|\eta_{21} \kappa_{1 i}^{\prime}+\eta_{22} \kappa_{2 i}^{\prime}+\sum_{j=1}^{T} w_{2 j} \kappa_{(j+2) i}^{\prime}\right|^{2}\right)\right]-h\left(\sum_{i=1}^{T}\left|\eta_{21} \kappa_{1 i}^{\prime}+\eta_{22} \kappa_{2 i}^{\prime}+\sum_{j=1}^{T} w_{2 j} \kappa_{(j+2) i}^{\prime}\right|^{2}\right)$

is uniformly bounded independent of $\kappa_{i j}^{\prime}$. We will show this by assuming $\left|\kappa_{11}^{\prime}\right|=\max _{i, j}\left(\left|\kappa_{i j}^{\prime}\right|\right)$.

This is without loss of generality since $\eta_{i j}, w_{i j}$ are all i.i.d. $\mathcal{C N}(0,1)$. Now

$$
\begin{aligned}
& \mathbb{E} {\left[\log \left(\sum_{i=1}^{T}\left|\eta_{21} \kappa_{1 i}^{\prime}+\eta_{22} \kappa_{2 i}^{\prime}+\sum_{j=1}^{T} w_{2 j} \kappa_{(j+2) i}^{\prime}\right|^{2}\right)\right]-h\left(\sum_{i=1}^{T}\left|\eta_{21} \kappa_{1 i}^{\prime}+\eta_{22} \kappa_{2 i}^{\prime}+\sum_{j=1}^{T} w_{2 j} \kappa_{(j+2) i}^{\prime}\right|^{2}\right) } \\
&= \mathbb{E}\left[\log \left(\sum_{i=1}^{T}\left|\eta_{21} \frac{\kappa_{1 i}^{\prime}}{\kappa_{11}^{\prime}}+\eta_{22} \frac{\kappa_{2 i}^{\prime}}{\kappa_{11}^{\prime}}+\sum_{j=1}^{T} w_{2 j} \frac{\kappa_{(j+2) i}^{\prime}}{\kappa_{11}^{\prime}}\right|^{2}\right)\right] \\
&-h\left(\sum_{i=1}^{T}\left|\eta_{21} \frac{\kappa_{1 i}^{\prime}}{\kappa_{11}^{\prime}}+\eta_{22} \frac{\kappa_{2 i}^{\prime}}{\kappa_{11}^{\prime}}+\sum_{j=1}^{T} w_{2 j} \frac{\kappa_{(j+2) i}^{\prime}}{\kappa_{11}^{\prime}}\right|^{2}\right) \\
& \stackrel{(i)}{\leq} \log \left(\sum_{i=1}^{T}\left[\left|\eta_{21} \frac{\kappa_{1 i}^{\prime}}{\kappa_{11}^{\prime}}+\eta_{22} \frac{\kappa_{2 i}^{\prime}}{\kappa_{11}^{\prime}}+\sum_{j=1}^{T} w_{2 j} \frac{\kappa_{(j+2) i}^{\prime}}{\kappa_{11}^{\prime}}\right|^{2}\right]\right) \\
& \quad-h\left(\sum_{i=1}^{T}\left|\eta_{21} \frac{\kappa_{1 i}^{\prime}}{\kappa_{11}^{\prime}}+\eta_{22} \frac{\kappa_{2 i}^{\prime}}{\kappa_{11}^{\prime}}+\sum_{j=1}^{T} w_{2 j} \frac{\kappa_{(j+2) i}^{\prime}}{\kappa_{11}^{\prime}}\right|^{2}\right) \\
& \stackrel{(i i)}{=} \log \left(\sum_{i=1}^{T}\left(\left|\frac{\kappa_{1 i}^{\prime}}{\kappa_{11}^{\prime}}\right|^{2}+\left|\frac{\kappa_{2 i}^{\prime}}{\kappa_{11}^{\prime}}\right|^{2}+\sum_{j=1}^{T}\left|\frac{\kappa_{(j+2) i}^{\prime}}{\kappa_{11}^{\prime}}\right|^{2}\right)\right)
\end{aligned}
$$




$$
\begin{gathered}
-h\left(\sum_{i=1}^{T}\left|\eta_{21} \frac{\kappa_{1 i}^{\prime}}{\kappa_{11}^{\prime}}+\eta_{22} \frac{\kappa_{2 i}^{\prime}}{\kappa_{11}^{\prime}}+\sum_{j=1}^{T} w_{2 j} \frac{\kappa_{(j+2) i}^{\prime}}{\kappa_{11}^{\prime}}\right|^{2}\right) \\
\stackrel{(i i i)}{\leq} \log (T(T+2))-h\left(\sum_{i=1}^{T}\left|\eta_{21} \frac{\kappa_{1 i}^{\prime}}{\kappa_{11}^{\prime}}+\eta_{22} \frac{\kappa_{2 i}^{\prime}}{\kappa_{11}^{\prime}}+\sum_{j=1}^{T} w_{2 j} \frac{\kappa_{(j+2) i}^{\prime}}{\kappa_{11}^{\prime}}\right|^{2}\right) \\
\stackrel{(i v)}{\leq} \log (T(T+2))-h\left(\sum_{i=1}^{T}\left|\eta_{21} \frac{\kappa_{1 i}^{\prime}}{\kappa_{11}^{\prime}}+\eta_{22} \frac{\kappa_{2 i}^{\prime}}{\kappa_{11}^{\prime}}+\sum_{j=1}^{T} w_{2 j} \frac{\kappa_{(j+2) i}^{\prime}}{\kappa_{11}^{\prime}}\right|^{2} \mid \eta_{22}, w_{2 j}\right)
\end{gathered}
$$

$\stackrel{(v)}{\leq} \log (T(T+2))+\frac{7}{2} \log (e)$,

where $(i)$ is using Jensen's inequality, $(i i)$ is using the fact that $\eta_{21} \frac{\kappa_{1 i}^{\prime}}{\kappa_{11}^{\prime}}+\eta_{22} \frac{\kappa_{2 i}^{\prime}}{\kappa_{11}^{\prime}}+\sum_{j=1}^{T} w_{2 j} \frac{\kappa_{(j+2) i}^{\prime}}{\kappa_{11}^{\prime}}$ is Complex Gaussian, (iii) is because $\frac{\left|\kappa_{i j}^{\prime}\right|}{\left|\kappa_{11}^{\prime}\right|} \leq 1$ since $\left|\kappa_{11}^{\prime}\right|=\max _{i, j}\left(\left|\kappa_{i j}^{\prime}\right|\right.$ ) (note that $\max _{i, j}\left(\left|\kappa_{i j}^{\prime}\right|\right) \geq 1$ for a valid set of $\kappa_{i j}^{\prime}$, due to the way $\kappa_{i j}$ is defined), (iv) is because conditioning reduces entropy and $(v)$ is by invoking the result from Appendix If.

Now if the term $\log (\mathbb{E}[V])-\mathbb{E}[\log (V)]$ does not approach infinity with the SNR then the desired result follows directly by choosing any fixed $\alpha>0$. When $\log (\mathbb{E}[V])-\mathbb{E}[\log (V)]$ does tend to infinity with SNR, following [7, (336)] we choose

$$
\alpha^{*}=(1+\log (\mathbb{E}[V])-\mathbb{E}[\log (V)])^{-1}
$$

with $\alpha^{*} \downarrow 0$ with the SNR and we have $\log \left(\Gamma\left(\alpha^{*}\right)\right)=\log \left(\frac{1}{\alpha^{*}}\right)+o(1)$ and $\alpha^{*} \log \left(\alpha^{*}\right)=o(1)$ where $o(1)$ tends to zero as $\alpha^{*}$ tends to zero, following [7, (337)]. Hence we have

$$
\begin{gathered}
I\left(\left|\xi_{22}\right|^{2} ; a, b, c,\left|\xi_{11}\right|^{2}\right) \leq\left(\log (T(T+2))+\frac{7}{2} \log (e)\right)+1+\log \left(\frac{1}{\alpha^{*}}\right)+o(1), \\
\frac{1}{\alpha^{*}}=1+\log \left(\mathbb{E}\left[\left|\xi_{22}\right|^{2}\right]\right)-\mathbb{E}\left[\log \left(\left|\xi_{22}\right|^{2}\right)\right] .
\end{gathered}
$$

Now

$$
\left|\xi_{22}\right|^{2} \leq\left|a g_{21}+b g_{22}+w_{21}\right|^{2}+\left|c g_{22}+w_{22}\right|^{2}+\sum_{i=3}^{T}\left|w_{2 i}\right|^{2}
$$

due to the LQ transformation on (44). Hence

$$
\begin{aligned}
\mathbb{E}\left[\left|\xi_{22}\right|^{2}\right] & \stackrel{(i)}{\leq} \mathbb{E}\left[\left(\rho_{21}^{2}|a|^{2}+\rho_{22}^{2}\left(|b|^{2}+|c|^{2}\right)+T\right)\right], \\
\log \left(\mathbb{E}\left[\left|\xi_{22}\right|^{2}\right]\right) & \stackrel{(i i)}{\leq} \log \left(\rho_{21}^{2}+\rho_{22}^{2}+1\right)+\log (T),
\end{aligned}
$$


where $(i)$ is using the fact that given $(a, b, c), a g_{21}+b g_{22}+w_{21}, c g_{22}+w_{22}$ are sums of independent Gaussians and $(i i)$ is using the power constraint on $a, b, c$. Hence we have

$$
\frac{1}{\alpha^{*}} \leq 1+\log \left(\rho_{21}^{2}+\rho_{22}^{2}+1\right)+\log (T)-\mathbb{E}\left[\log \left(\left|\xi_{22}\right|^{2}\right)\right] .
$$

Now we lower bound $\mathbb{E}\left[\log \left(\left|\xi_{22}\right|^{2}\right)\right]$. Note that

$$
\begin{aligned}
\left|\xi_{22}\right|^{2}= & \left|a g_{21}+b g_{22}+w_{21}\right|^{2}+\left|c g_{22}+w_{22}\right|^{2}+\sum_{i=3}^{T}\left|w_{2 i}\right|^{2} \\
& -\frac{\left|\left(a g_{21}+b g_{22}+w_{21}\right)\left(a g_{11}+b g_{12}+w_{11}\right)^{*}+\left(c g_{22}+w_{22}\right)\left(c g_{12}+w_{12}\right)^{*}+\sum_{i=3}^{T} w_{2 i} w_{1 i}^{*}\right|^{2}}{\left|a g_{11}+b g_{12}+w_{11}\right|^{2}+\left|c g_{12}+w_{12}\right|^{2}+\sum_{i=3}^{T}\left|w_{1 i}\right|^{2}}
\end{aligned}
$$

is the magnitude squared of the projection of the Complex vector

$$
\left[\left(a g_{21}+b g_{22}+w_{21}\right),\left(c g_{22}+w_{22}\right), w_{23}, \ldots, w_{2 T}\right]
$$

onto the subspace orthogonal to the Complex vector

$$
\left[\left(a g_{11}+b g_{12}+w_{11}\right),\left(c g_{12}+w_{12}\right), w_{13}, \ldots, w_{1 T}\right] .
$$

Note that $\left[\left(c g_{12}+w_{12}\right)^{*},-\left(a g_{11}+b g_{12}+w_{11}\right)^{*}, 0, \ldots, 0\right]$ is orthogonal to

$$
\left[\left(a g_{11}+b g_{12}+w_{11}\right),\left(c g_{12}+w_{12}\right), w_{13}, \ldots, w_{1 T}\right] .
$$

Hence

$$
\begin{aligned}
& \left|\xi_{22}\right|^{2} \geq \frac{\left|\left[\begin{array}{lllll}
a g_{21}+b g_{22}+w_{21}, & c g_{22}+w_{22}, & w_{23}, & \ldots, & w_{2 T}
\end{array}\right]\left[\begin{array}{c}
c g_{12}+w_{12} \\
-\left(a g_{11}+b g_{12}+w_{11}\right) \\
0 \\
\vdots \\
0
\end{array}\right]\right|^{2}}{\left|a g_{11}+b g_{12}+w_{11}\right|^{2}+\left|c g_{12}+w_{12}\right|^{2}} \\
& =\frac{\left|\left(a g_{21}+b g_{22}+w_{21}\right)\left(c g_{12}+w_{12}\right)-\left(c g_{22}+w_{22}\right)\left(a g_{11}+b g_{12}+w_{11}\right)\right|^{2}}{\left|a g_{11}+b g_{12}+w_{11}\right|^{2}+\left|c g_{12}+w_{12}\right|^{2}}
\end{aligned}
$$

and hence

$$
\begin{aligned}
& \mathbb{E}\left[\log \left(\left|\xi_{22}\right|^{2}\right)\right] \\
& \geq \mathbb{E}\left[\log \left(\frac{\left|\left(a g_{21}+b g_{22}+w_{21}\right)\left(c g_{12}+w_{12}\right)-\left(c g_{22}+w_{22}\right)\left(a g_{11}+b g_{12}+w_{11}\right)\right|^{2}}{\left|a g_{11}+b g_{12}+w_{11}\right|^{2}+\left|c g_{12}+w_{12}\right|^{2}}\right)\right]
\end{aligned}
$$




$$
=\mathbb{E}\left[\log \left(\left|\left(a g_{21}+b g_{22}+w_{21}\right) u_{1}^{\prime}-\left(c g_{22}+w_{22}\right) u_{2}^{\prime}\right|^{2}\right)\right],
$$

where $\left(u_{1}^{\prime}, u_{2}^{\prime}\right)$ is a unit norm complex vector independent of $g_{2 i}, w_{2 i}$. Hence

$$
\begin{aligned}
& \mathbb{E}\left[\log \left(\left|\xi_{22}\right|^{2}\right)\right] \\
& \stackrel{(i)}{\geq} \mathbb{E}\left[\log \left(\left|a u_{1}^{\prime}\right|^{2} \rho_{21}^{2}+\left|b u_{1}^{\prime}-c u_{2}^{\prime}\right|^{2} \rho_{22}^{2}+\left|u_{1}^{\prime}\right|^{2}+\left|u_{2}^{\prime}\right|^{2}\right)\right]-\gamma_{E} \log (e) \\
& \stackrel{(i i)}{\geq} \mathbb{E}\left[\log \left(\left|a u_{1}^{\prime}\right|^{2} \rho_{21}^{2}+\left|b u_{1}^{\prime}-c u_{2}^{\prime}\right|^{2} \rho_{22}^{2}+1\right)\right]-\gamma_{E} \log (e) \\
& \geq-\gamma \log \left({ }_{E} e\right)
\end{aligned}
$$

where $(i)$ is using the fact that given $\left(a, b, c, u_{1}^{\prime}, u_{2}^{\prime}\right),\left(a g_{21}+b g_{22}+w_{21}\right) u_{1}^{\prime}-\left(c g_{22}+w_{22}\right) u_{2}^{\prime}$ is Complex Gaussian distributed with variance $\left|a u_{1}^{\prime}\right|^{2} \rho_{21}^{2}+\left|b u_{1}^{\prime}-c u_{2}^{\prime}\right|^{2} \rho_{22}^{2}+\left|u_{1}^{\prime}\right|^{2}+\left|u_{2}^{\prime}\right|^{2}$ and applying Lemma 9 on page 16 together with Tower property of expectation. The step (ii) is because $\left(u_{1}^{\prime}, u_{2}^{\prime}\right)$ is a unit norm vector.

Substituting 280) in (271) we get

$$
\begin{aligned}
\frac{1}{\alpha^{*}} & \leq \log \left(\rho_{21}^{2}+\rho_{22}^{2}+1\right)+1+\log (T)+\gamma_{E} \log (e) \\
& =\log \left(\rho_{21}^{2}+\rho_{22}^{2}+1\right)+r_{2}(T)
\end{aligned}
$$

and hence by substituting the above in 266, we get

$$
\begin{aligned}
I\left(\left|\xi_{22}\right|^{2} ; a, b, c,\left|\xi_{11}\right|^{2}\right) \leq & \left(\log (T(T+2))+\frac{7}{2} \log (e)\right)+1 \\
& +\log \left(\log \left(\rho_{21}^{2}+\rho_{22}^{2}+1\right)+r_{2}(T)\right)+o(1)
\end{aligned}
$$

where $r_{2}(T)=1+\log (T)+\gamma_{E} \log (e)$ is a function of $T$ alone. Hence $I\left(\left|\xi_{22}\right|^{2} ; a, b, c,\left|\xi_{11}\right|^{2}\right)$ has zero gDoF. Now since

$$
I\left(\left|\xi_{22}\right|^{2} ; a, b,\left.c|| \xi_{11}\right|^{2}\right) \leq I\left(\left|\xi_{22}\right|^{2} ; a, b, c,\left|\xi_{11}\right|^{2}\right),
$$

it follows that $h\left(\left.\left|\xi_{22}\right|^{2}|| \xi_{11}\right|^{2}\right) \doteq h\left(\left.\left|\xi_{22}\right|^{2}|| \xi_{11}\right|^{2}, a, b, c\right)$.

\section{APPENDIX I}

\section{A LOWER BOUND ON ENTROPY OF SQUARED 2-NORM OF A GAUSSIAN VECTOR}

For complex $l_{i}, k_{i}, l$ for finite number of $i$ 's with $\left|k_{i}\right| \leq 1$ and $\eta \sim \mathcal{C N}(0,1)$ we will show that

$$
h\left(|\eta+l|^{2}+\sum_{i}\left|k_{i} \eta+l_{i}\right|^{2}\right) \geq-\frac{7}{2} \log (e) .
$$


We have

$$
\begin{aligned}
& h\left(|\eta+l|^{2}+\sum_{i}\left|k_{i} \eta+l_{i}\right|^{2}\right) \\
& =h\left(|l|^{2}+2 \operatorname{Re}\left(\left(l^{*}+\sum_{i} l_{i}^{*} k_{i}\right) \eta\right)+|\eta|^{2}\left(1+\sum_{i}\left|k_{i}\right|^{2}\right)\right) \\
& =h\left(\left|\eta \sqrt{1+\sum_{i}\left|k_{i}\right|^{2}}+\frac{l+\sum_{i} l_{i} k_{i}^{*}}{\sqrt{1+\sum_{i}\left|k_{i}\right|^{2}}}\right|^{2}\right)
\end{aligned}
$$

Now it suffices to show that $h\left(\left|\eta k^{\prime}+l^{\prime}\right|^{2}\right)>-(7 / 2) \log (e)$ for $\left|k^{\prime}\right| \geq 1$. Now,

$$
h\left(\left|\eta k^{\prime}+l^{\prime}\right|^{2}\right)=h\left(\left|\eta k^{\prime}\right|^{2}+2|\eta|\left|k^{\prime}\right|\left|l^{\prime}\right| \cos \theta+\left|l^{\prime}\right|^{2}\right)
$$

where $\theta$ is uniformly distributed in $[0,2 \pi]$ and is independent of $|\eta|$ since $\eta$ is circularly symmetric Gaussian.

$$
\begin{aligned}
h\left(\left|\eta k^{\prime}+l^{\prime}\right|^{2}\right) & \geq h\left(\left|\eta k^{\prime}\right|^{2}+2|\eta|\left|k^{\prime}\right|\left|l^{\prime}\right| \cos \theta+\left|l^{\prime}\right|^{2} \mid \theta\right) \\
& =h\left(\left(\left|\eta k^{\prime}\right|+\left|l^{\prime}\right| \cos \theta\right)^{2} \mid \theta\right)
\end{aligned}
$$

Consider $S=\| \eta|| k^{\prime}|+| l^{\prime}\left|\cos \theta^{\prime}\right|$ for a constant $\theta^{\prime}$. It suffices to show that $h\left(S^{2}\right) \geq$ $-(7 / 2) \log (e)$ to complete the proof. Now $\eta^{\prime}=|\eta|\left|k^{\prime}\right|$ is Rayleigh distributed with probability density function $p_{\eta^{\prime}}(x)=\left(x /\left|k^{\prime}\right|^{2}\right) \exp \left(-x^{2} /\left(2\left|k^{\prime}\right|^{2}\right)\right)$ and it easily follows that $p_{\eta^{\prime}}(x) \leq$ $\left(1 /\left|k^{\prime}\right|\right) \exp (-1 / 2) \leq \exp (-1 / 2)$ since $\left|k^{\prime}\right| \geq 1$. Hence the probability density function of $S$ has $p_{s}(x) \leq 2 \exp (-1 / 2)$. Hence

$$
\begin{aligned}
h(S) & =-\mathbb{E}\left[\log \left(p_{s}(S)\right)\right] \\
& \geq-\log \left(2 e^{-\frac{1}{2}}\right)
\end{aligned}
$$

Using [7, (316)] for rates in bits, we have

$$
\begin{aligned}
h\left(S^{2}\right) & =h(S)+\mathbb{E}[\log (S)]+1 \\
& \geq-\log \left(2 e^{-\frac{1}{2}}\right)+\mathbb{E}\left[\log \left(|| \eta|| k^{\prime}|+| l^{\prime}\left|\cos \theta^{\prime}\right|\right)\right]+1 \\
& =\frac{1}{2} \log (e)+\mathbb{E}\left[\log \left(|| \eta|| k^{\prime}|+| l^{\prime}\left|\cos \theta^{\prime}\right|\right)\right]
\end{aligned}
$$


Now it suffices to show that $\mathbb{E}\left[\log \left(|\eta|\left|k^{\prime}\right|+\left|l^{\prime}\right| \cos \theta^{\prime} \mid\right)\right]$ is lower bounded by $-4 \log (e)$ to complete the proof. For a random variable $X$ we define $h^{-}(X)=\int_{p(x)>1} p(x) \log (p(x)) d x$. We have

$$
\begin{aligned}
h^{-}\left(|\eta|\left|k^{\prime}\right|\right) & =\int_{p_{\eta^{\prime}}(x)>1} p_{\eta^{\prime}}(x) \log \left(p_{\eta^{\prime}}(x)\right) d x \\
& =0
\end{aligned}
$$

since $p_{\eta^{\prime}}(x) \leq\left(1 /\left|k^{\prime}\right|\right) \exp (-1 / 2) \leq \exp (-1 / 2)$. Using [7, (257)] to bound the expected $\operatorname{logarithm}\left(\mathbb{E}[\log (|X|)] \geq-\frac{1}{(1-\alpha)^{2}} \log (e)-\frac{1}{\alpha} h^{-}(X)\right.$ with $h^{-}(X)=\int_{p(x)>1} p(x) \log (p(x)) d x$ for any $0<\alpha<1$ ), we have

$$
\begin{aligned}
& \mathbb{E}[\log (|X|)] \geq-\frac{1}{(1-\alpha)^{2}} \log (e)-\frac{1}{\alpha} h^{-}(X), 0<\alpha<1, \\
& \mathbb{E}\left[\log \left(|| \eta|| k^{\prime}|+| l^{\prime}\left|\cos \theta^{\prime}\right|\right)\right] \stackrel{(i)}{\geq}-\frac{1}{\left(1-\frac{1}{2}\right)^{2}} \log (e)-2 h^{-}\left(|\eta|\left|k^{\prime}\right|+\left|l^{\prime}\right| \cos \theta^{\prime}\right) \\
&=-2 h^{-}\left(|\eta|\left|k^{\prime}\right|\right)-4 \log (e) \\
& \stackrel{(i i)}{=} 0-4 \log (e),
\end{aligned}
$$

where $(i)$ is using [7, (257)] with $\alpha=\frac{1}{2}$ and $(i i)$ is using 296. Now using 300 in 294 the proof is complete.

\section{APPENDIX $\mathbf{J}$}

\section{Numerical CALCULATION OF INNER BOUND FOR $T=2$}

Here we provide the calculations required for numerically evaluating the achievable rates given in Table III. We consider the case with $T=2$. In the calculations below, the channels are scaled, so that the average power per transmit symbol from each antenna is unity. Also, Gaussian codebooks are used in the training based schemes.

\section{A. Training Scheme Using Only One Antenna}

For a training-based scheme using only one antenna (reducing to a SISO case), we use one symbol (of value 1) to train the channel to obtain $Y_{1, \text { train }}=g_{11}+w$ at the receiver. The minimum mean squared error (MMSE) estimate for the channel is

$$
\hat{g}_{11}=\frac{\mathbb{E}\left[\left|g_{11}\right|^{2}\right]}{1+\mathbb{E}\left[\left|g_{11}\right|^{2}\right]} Y_{1, \text { train }}
$$




$$
\mathbb{E}\left[\left|g_{11}\right|^{2}\right] \frac{g_{11}+w}{1+\mathbb{E}\left[\left|g_{11}\right|^{2}\right]} .
$$

The total noise including MMSE is

$$
\begin{aligned}
N_{\text {SISO }}= & \mathbb{E}\left[\left(g_{11}-\mathbb{E}\left[\left|g_{11}\right|^{2}\right] \frac{g_{11}+w}{1+\mathbb{E}\left[\left|g_{11}\right|^{2}\right]}\right)^{2}\right]+1 \\
= & \mathbb{E}\left[\left|\frac{g_{11}}{1+\mathbb{E}\left[\left|g_{11}\right|^{2}\right]}\right|^{2}\right]+\frac{\mathbb{E}\left[\left|g_{11}\right|^{2}\right]^{2}}{\left|1+\mathbb{E}\left[\left|g_{11}\right|^{2}\right]\right|^{2}}+1 \\
= & \frac{\mathbb{E}\left[\left|g_{11}\right|^{2}\right]}{1+\mathbb{E}\left[\left|g_{11}\right|^{2}\right]}+1
\end{aligned}
$$

and after scaling with $T=2$, the achievable rate is calculated as

$$
R_{\text {SISO }}=\frac{1}{2} \mathbb{E}\left[\log \left(1+\frac{\left|\hat{g}_{11}\right|^{2}}{N_{\text {SISO }}}\right)\right] .
$$

\section{B. Training Scheme Using Both Antennas}

If we treat the system as a parallel antenna system, treating the crosslinks as noise and use one symbol to train the channel, then we get $Y_{1, \text { train }}=g_{11}+g_{12}+w$ at the first receiver antenna. The MMSE estimate for the channel to the first antenna is

$$
\begin{gathered}
\hat{g}_{11}=\frac{\mathbb{E}\left[\left|g_{11}\right|^{2}\right]}{1+\mathbb{E}\left[\left|g_{11}\right|^{2}\right]+\mathbb{E}\left[\left|g_{12}\right|^{2}\right]} Y_{1, \text { train }} \\
=\mathbb{E}\left[\left|g_{11}\right|^{2}\right] \frac{g_{11}+g_{12}+w}{1+\mathbb{E}\left[\left|g_{11}\right|^{2}\right]+\mathbb{E}\left[\left|g_{12}\right|^{2}\right]} .
\end{gathered}
$$

The total noise including MMSE is

$$
\begin{aligned}
N_{\text {Parallel }}= & \mathbb{E}\left[\left(g_{11}-\mathbb{E}\left[\left|g_{11}\right|^{2}\right] \frac{g_{11}+g_{12}+w}{1+\mathbb{E}\left[\left|g_{11}\right|^{2}\right]+\mathbb{E}\left[\left|g_{12}\right|^{2}\right]}\right)^{2}\right]+1+\mathbb{E}\left[\left|g_{12}\right|^{2}\right] \\
= & \mathbb{E}\left[\left|\frac{g_{11}}{1+\mathbb{E}\left[\left|g_{11}\right|^{2}\right]+\mathbb{E}\left[\left|g_{12}\right|^{2}\right]}\right|^{2}\left(1+\mathbb{E}\left[\left|g_{12}\right|^{2}\right]\right)^{2}\right]+\frac{\mathbb{E}\left[\left|g_{11}\right|^{2}\right]^{2}\left(1+\mathbb{E}\left[\left|g_{12}\right|^{2}\right]\right)}{\left|1+\mathbb{E}\left[\left|g_{11}\right|^{2}\right]+\mathbb{E}\left[\left|g_{12}\right|^{2}\right]\right|^{2}} \\
& +1+\mathbb{E}\left[\left|g_{12}\right|^{2}\right] \\
= & \frac{\mathbb{E}\left[\left|g_{11}\right|^{2}\right]\left(1+\mathbb{E}\left[\left|g_{12}\right|^{2}\right]\right)^{2}+\mathbb{E}\left[\left|g_{11}\right|^{2}\right]^{2}\left(1+\mathbb{E}\left[\left|g_{12}\right|^{2}\right]\right)}{\left|1+\mathbb{E}\left[\left|g_{11}\right|^{2}\right]+\mathbb{E}\left[\left|g_{12}\right|^{2}\right]\right|^{2}}+1+\mathbb{E}\left[\left|g_{12}\right|^{2}\right] \\
= & \frac{\mathbb{E}\left[\left|g_{11}\right|^{2}\right]\left(1+\mathbb{E}\left[\left|g_{12}\right|^{2}\right]\right)}{1+\mathbb{E}\left[\left|g_{11}\right|^{2}\right]+\mathbb{E}\left[\left|g_{12}\right|^{2}\right]}+1+\mathbb{E}\left[\left|g_{12}\right|^{2}\right]
\end{aligned}
$$


and using symmetry, the achievable rate (after scaling with $T=2$ ) using both antennas is calculated as

$$
R_{\text {Parallel }}=\mathbb{E}\left[\log \left(1+\frac{\left|\hat{g}_{11}\right|^{2}}{N_{\text {Parallel }}}\right)\right]
$$

\section{Noncoherent Scheme}

We evaluate the mutual information carefully for $T=2$ case for numerically calculating it. Using the input distribution as given in Theorem 7, we have

$$
\begin{aligned}
& I(X ; Y)=h(Y)-h(Y \mid X) \\
& h(Y)=h(G X+W) \\
& =h\left(\left[\begin{array}{ll}
g_{11} & g_{12} \\
g_{21} & g_{22}
\end{array}\right]\left[\begin{array}{ll}
a & 0 \\
\eta & c
\end{array}\right] Q+W\right) \\
& =h\left(\left[\begin{array}{ll}
a g_{11}+\eta g_{12} & c g_{12} \\
a g_{21}+\eta g_{22} & c g_{22}
\end{array}\right] Q+W\right) \\
& \stackrel{(i)}{=} h\left(\left(\left[\begin{array}{ll}
a g_{11}+\eta g_{12} & c g_{12} \\
a g_{21}+\eta g_{22} & c g_{22}
\end{array}\right]+W\right) Q\right) \text {, }
\end{aligned}
$$

where $(i)$ is using the fact that $W$ and $W Q$ have the same distribution. Now

$$
\begin{aligned}
& h(Y)=h\left(\left(\left[\begin{array}{ll}
a g_{11}+\eta g_{12} & c g_{12} \\
a g_{21}+\eta g_{22} & c g_{22}
\end{array}\right]+W\right) Q\right) \\
& \stackrel{(i)}{=} h\left(\left[\begin{array}{ll}
a g_{11}+\eta g_{12}+w_{1} & c g_{12}+w_{2} \\
a g_{21}+\eta g_{22}+w_{3} & c g_{22}+w_{4}
\end{array}\right] Q\right) \\
& \stackrel{(i i)}{\geq} h\left(\left[a g_{11}+\eta g_{12}+w_{1}, \quad c g_{12}+w_{2}\right] Q\right) \\
& +h\left(\left[a g_{21}+\eta g_{22}+w_{3}, c g_{22}+w_{4}\right] Q \mid Q, a g_{11}+\eta g_{12}+w_{1}, c g_{12}+w_{2}\right) \\
& \stackrel{(i i i)}{=} h\left(\left|a g_{11}+\eta g_{12}\right|^{2}+\left|c g_{12}\right|^{2}\right)+\mathbb{E}\left[\log \left(\left|a g_{11}+\eta g_{12}\right|^{2}+\left|c g_{12}\right|^{2}\right)\right]+\log \left(\frac{\pi^{T}}{\Gamma(T)}\right) \\
& +h\left(\left[a g_{21}+\eta g_{22}+w_{3}, c g_{22}+w_{4}\right] Q \mid Q, a g_{11}+\eta g_{12}+w_{1}, c g_{12}+w_{2}\right),
\end{aligned}
$$

where in step $(i), w_{i}$ 's are i.i.d $\mathcal{C N}(0,1)$ and the step $(i i)$ is using the fact that conditioning reduces entropy. The step (iii) is using Lemma 13 . Now

$$
h\left(\left[a g_{21}+\eta g_{22}+w_{3}, c g_{22}+w_{4}\right] Q \mid Q, a g_{11}+\eta g_{12}+w_{1}, c g_{12}+w_{2}\right)
$$




$$
\begin{aligned}
& =h\left(\left[a g_{21}+\eta g_{22}+w_{3}, \quad c g_{22}+w_{4}\right] \mid a g_{11}+\eta g_{12}+w_{1}, c g_{12}+w_{2}\right) \\
& \stackrel{(i)}{=} h\left(c g_{22}+w_{4}\right)+h\left(a g_{21}+\eta g_{22}+w_{3} \mid a g_{11}+\eta g_{12}+w_{1}, c g_{12}+w_{2}\right) \\
& \stackrel{(i i)}{\geq} h\left(c g_{22}+w_{4}\right)+h\left(a g_{21}+\eta g_{22} \mid c g_{22}, a g_{11}+\eta g_{12}, c g_{12}\right),
\end{aligned}
$$

where $(i)$ is using the fact that $c$ is a given constant and $c g_{22}+w_{4}$ is independent of $a g_{11}+$ $\eta g_{12}+w_{1}, c g_{12}+w_{2}$. The step $(i i)$ is by providing $w_{1}, w_{2}, w_{3}$ in the conditioning and using the fact that conditioning reduces entropy. Hence

$$
\begin{gathered}
h(Y) \geq h\left(\left|a g_{11}+\eta g_{12}\right|^{2}+\left|c g_{12}\right|^{2}\right)+\mathbb{E}\left[\log \left(\left|a g_{11}+\eta g_{12}\right|^{2}+\left|c g_{12}\right|^{2}\right)\right]+\log \left(\frac{\pi^{T}}{\Gamma(T)}\right) \\
h\left(c g_{22}+w_{4}\right)+h\left(a g_{21}+\eta g_{22} \mid c g_{22}, a g_{11}+\eta g_{12}, c g_{12}\right) .
\end{gathered}
$$

Now for numerically evaluating the terms in the above expression, we use the following:

$$
h\left(\left|a g_{11}+\eta g_{12}\right|^{2}+\left|c g_{12}\right|^{2}\right) \geq h\left(\left|a g_{11}+\eta g_{12}\right|^{2} \mid g_{12}\right)
$$

and

$\mathbb{E}\left[\log \left(\left|a g_{11}+\eta g_{12}\right|^{2}+\left|c g_{12}\right|^{2}\right)\right]$

$$
\begin{aligned}
& \stackrel{(i)}{=} \mathbb{E}\left[\log \left(|a|^{2}\left|g_{11}\right|^{2}+|\eta|^{2}\left|g_{12}\right|^{2}+|c|^{2}\left|g_{12}\right|^{2}+2\left|a \eta g_{12} g_{11}\right| \cos \theta\right)\right] \\
& \stackrel{(i i)}{=} \mathbb{E}\left[\log \left(\frac{|a|^{2}\left|g_{11}\right|^{2}+|\eta|^{2}\left|g_{12}\right|^{2}+|c|^{2}\left|g_{12}\right|^{2}+\sqrt{\left.|| a\right|^{2}\left|g_{11}\right|^{2}+|\eta|^{2}\left|g_{12}\right|^{2}+\left.|c|^{2}\left|g_{12}\right|^{2}\right|^{2}-4\left|a \eta g_{12} g_{11}\right|}}{2}\right)\right]
\end{aligned}
$$

where $(i)$ is using a $\theta$ uniformly distributed in $[0,2 \pi]$ and the fact that $g_{12}, g_{11}, \eta$ are independent circularly symmetric Gaussians. The step $(i i)$ is by evaluating the expectation over $\theta$. Also $h\left(a g_{21}+\eta g_{22} \mid c g_{22}, a g_{11}+\eta g_{12}, c g_{12}\right)$ is evaluated using (153) on page 44. Also, using (24), (28) we have

$$
\begin{aligned}
& h(Y \mid X) \\
&= \mathbb{E}\left[\log \left(|a|^{2} \rho_{11}^{2}+|\eta|^{2} \rho_{12}^{2}+|c|^{2} \rho_{12}^{2}+|a|^{2}|c|^{2} \rho_{11}^{2} \rho_{12}^{2}+1\right)\right] \\
&+\mathbb{E}\left[\log \left(|a|^{2} \rho_{21}^{2}+|\eta|^{2} \rho_{22}^{2}+|c|^{2} \rho_{22}^{2}+|a|^{2}|c|^{2} \rho_{21}^{2} \rho_{22}^{2}+1\right)\right] \\
&+2 T \log (\pi e) .
\end{aligned}
$$

Now following the choice in Theorem 7, we choose

$$
|a|^{2}=2, \eta \sim \mathcal{C N}\left(0,|b|^{2}\right),|b|^{2}=1,|c|^{2}=1 / \rho_{12}^{2}
$$


and evaluate the achievable rate using (314) to $(316)$ and scaling with $T=2$. Note that the expressions given here assume that the transmit SNR is scaled into the link strengths $\rho_{i j}^{2}$ and the power at the antennas are unity after the scaling. 